Draft Version June 30, 2021

Preprint typeset using $\mathrm{I}_{\mathrm{T}} \mathrm{EX}$ style emulateapj v. 04/17/13

\title{
MODELLING THE MOLECULAR GAS IN NGC 6240
}

\author{
R. Tunnard ${ }^{1}$, T. R. Greve ${ }^{1}$, S. Garcia-Burillo ${ }^{2}$, J. Graciá Carpio ${ }^{3}$, A. Fuente ${ }^{2}$, L. Tacconi $^{3}$, R. Neri $^{4}$ And A. \\ $\mathrm{USERO}^{2}$. \\ Draft version June 30, 2021
}

\begin{abstract}
We present the first observations of $\mathrm{H}^{13} \mathrm{CN}(1-0), \mathrm{H}^{13} \mathrm{CO}^{+}(1-0)$ and $\mathrm{SiO}(2-1)$ in $\mathrm{NGC} 6240$, obtained with the IRAM PdBI. Combining a Markov Chain Monte Carlo (MCMC) code with Large Velocity Gradient (LVG) modelling, and with additional data from the literature, we simultaneously fit three gas phases and six molecular species to constrain the physical condition of the molecular gas, including mass-luminosity conversion factors. We find $\sim 10^{10} M_{\odot}$ of dense molecular gas in cold, dense clouds $\left(T_{\mathrm{k}} \sim 10 \mathrm{~K}, n_{\mathrm{H}_{2}} \sim 10^{6} \mathrm{~cm}^{-3}\right)$ with a volume filling factor $<0.002$, embedded in a shock heated molecular medium $\left(T_{\mathrm{k}} \sim 2000 \mathrm{~K}, n_{\mathrm{H}_{2}} \sim 10^{3.6} \mathrm{~cm}^{-3}\right)$, both surrounded by an extended diffuse phase $\left(T_{\mathrm{k}} \sim 200 \mathrm{~K}, n_{\mathrm{H}_{2}} \sim 10^{2.5} \mathrm{~cm}^{-3}\right)$. We derive a global $\alpha_{\mathrm{CO}}=1.5_{1.1}^{7.1}$ with gas masses $\log _{10}\left(M /\left[M_{\odot}\right]\right)=10.1_{10.0}^{10.8}$, dominated by the dense gas. We also find $\alpha_{\mathrm{HCN}}=32_{13}^{89}$, which traces the cold, dense gas. The $\left[{ }^{12} \mathrm{C}\right] /\left[{ }^{13} \mathrm{C}\right]$ ratio is only slightly elevated $\left(98_{65}^{230}\right)$, contrary to the very high $[\mathrm{CO}] /\left[{ }^{13} \mathrm{CO}\right]$ ratio $(300-500)$ reported in the literature. However, we find very high $[\mathrm{HCN}] /\left[\mathrm{H}^{13} \mathrm{CN}\right]$ and $\left[\mathrm{HCO}^{+}\right] /\left[\mathrm{H}^{13} \mathrm{CO}^{+}\right]$abundance ratios $\left(300_{200}^{500}\right)$ which we attribute to isotope fractionation in the cold, dense clouds.
\end{abstract}

\section{INTRODUCTION}

The term "dense gas" typically refers to molecular gas with $n_{\mathrm{H}_{2}}>10^{4} \mathrm{~cm}^{-3}$, which is the typical density of molecular cloud cores bound by their self-gravity (e.g., Lada 1992). This phase offers significant shielding against photo-dissociation while being heated and partially ionised by cosmic rays, leading to chemistry distinct from elsewhere in the ISM. It is best traced by the rotational transitions of molecules such as $\mathrm{HCN}, \mathrm{HCO}^{+}$, and CS, which can reach high abundances relative to $\mathrm{H}_{2}$ $\left(10^{-8}-10^{-6}\right)$ under these conditions (Nguyen et al. 1992; Solomon et al. 1992; Boger \& Sternberg 2005). Due to both high densities and line trapping the low $-J$ transitions of these lines (in particular $\mathrm{HCN}$ ) are almost exclusively optically thick, so that approaches to estimating gas mass developed for CO (Young \& Scoville 1982; Papadopoulos et al. 2012; Bolatto et al. 2013) become applicable, with appropriate calibrations, allowing us to trace specifically dense molecular gas.

Single-dish surveys of primarily the $\mathrm{HCN}$, but also the $\mathrm{HCO}^{+}, J=1-0$ line towards samples of local (ultra)luminous infrared galaxies ((U)LIRGs) suggest that a larger fraction of the molecular gas in these galaxies is in a dense state compared to that of normal star forming galaxies (Solomon et al. 1992; Gao \& Solomon 2004; García-Burillo et al. 2012), where the $\mathrm{HCN} / \mathrm{CO} J=1-0$ luminosity ratio is adopted as a proxy of the dense gas mass fraction (typically $\sim 0.1$ in LIRGs/ULIRGs cf. $\sim 0.03$ in normal star forming galaxies, Gao \& Solomon 2004; Greve et al. 2014). However, to date, only a hand-

\footnotetext{
${ }^{1}$ Department of Physics and Astronomy, University College London, Gower Street, London WC1E 6BT, UK; richard.tunnard.13@ucl.ac.uk

2 Observatorio Astronómico Nacional, Observatorio de Madrid, Alfonso XII, 3, 28014 Madrid, Spain

${ }^{3}$ Max-Planck-Institute for Extraterrestrial Physics (MPE), Giessenbachstraße 1, 85748 Garching, Germany

${ }^{4}$ IRAM, 300 rue de la Piscine, Domaine Universitaire, 38406 Saint Martin d'Hères Cedex, France
}

ful of sources have been analysed using multi-line datasets of dense gas tracers, in conjunction with radiative transfer analyses, to constrain the dense gas mass (Krips et al. 2008; Greve et al. 2009; Papadopoulos et al. 2014).

Gas mass conversion factors typically assume: 1) something about the kinematical state of the gas; usually that it resides within virialized clouds (Bolatto et al. 2013, although see Papadopoulos et al. 2012 for a formulation that allows for a generalised kinematic state): perhaps not unreasonable for dense gas tracers but certainly questionably for CO. 2) that the lines are optically thick, which is generally a good assumption, at least for the $J=1-0$ lines. 3) metallicity and/or molecular abundances. These are particularly uncertain, being strongly affected by the environment the emission is tracing, e.g., photon dominated regions (PDRs), Xray dominated regions (XDRs), hot-cores or cold, dark clouds, and are usually a subject of interest in themselves. There are also possible contaminations from other sources, such as mid-IR pumping of HCN (and perhaps $\mathrm{HNC}$ and $\mathrm{HCO}^{+}$), which increases the low- $J \mathrm{HCN}$ line intensity (Aalto et al. 2007, 2012, 2015). As we move to higher redshifts the effect of the CMB becomes noticeably non-linear, with the greatest effects on the low- $J$ $\mathrm{CO}$ lines, and even in the local universe bright continuum backgrounds could be having significant effects on observed lines and line ratios (Papadopoulos et al. 2000, 2010). $\mathrm{HCO}^{+}$is subject to a complicated network of ion chemistry and photochemical reactions, making it particularly complicated molecular tracer (Viti et al. 2002; Papadopoulos 2007).

Optically thin dense gas tracers, in particular isotopologues of the main species (e.g., $\mathrm{H}^{13} \mathrm{CN}$ and $\mathrm{H}^{13} \mathrm{CO}^{+}$), can be especially powerful as they provide much tighter constraints on large velocity gradient (LVG) models when combined with their ${ }^{12} \mathrm{C}$ isotopologues. Although usually $40-100 \times$, and perhaps up to $\sim 500 \times$, less abundant than their ${ }^{12} \mathrm{C}$ isotopologues, optical depth effects 
mean that these lines are usually only $\sim 10-15 \times$ fainter, and are detectable with the IRAM Plateau de Bure Interferometer $(\mathrm{PdBI})$ and certainly with the Atacama Large Millimeter/submillimeter Array (ALMA) for the closest LIRGs and ULIRGs.

\subsection{Chemical Modelling and Isotopologue Ratios}

If it can be accurately measured, the elemental $\left[{ }^{12} \mathrm{C}\right] /\left[{ }^{13} \mathrm{C}\right]$ abundance ratio can be a powerful diagnostic of the evolutionary state of a galaxy, with a high $\left[{ }^{12} \mathrm{C}\right] /\left[{ }^{13} \mathrm{C}\right]$ being evidence for a young, unprocessed ISM (Milam et al. 2005; Henkel et al. 2010). Recently, it has been suggested by Papadopoulos et al. (2011) that a high $\left[{ }^{12} \mathrm{C}\right] /\left[{ }^{13} \mathrm{C}\right]$ ratio might be evidence for a cosmic ray dominated star formation paradigm, leading to a top heavy stellar initial mass function and thereby an excess of massive, ${ }^{12} \mathrm{C}$ producing, stars. Typical values of $\left[{ }^{12} \mathrm{C}\right] /\left[{ }^{13} \mathrm{C}\right]$ in the local universe are $\sim 25$ in the central Milky Way, 68 in the Milky Way as a whole and up to $\sim 100$ in local starbursts and $\gtrsim 100$ in high redshift ULIRGs (Milam et al. 2005; Henkel et al. 2010, 2014). Typically, these ratios are measured using a combination of observations of $\mathrm{CN},{ }^{13} \mathrm{CN}, \mathrm{CO},{ }^{13} \mathrm{CO}$ and $\mathrm{C}^{18} \mathrm{O}$ lines. However, this measurement is non-trivial due to chemical effects leading to isotope fractionation under a variety of circumstances, as well as optical depth effects.

The theory of ${ }^{12} \mathrm{C} /{ }^{13} \mathrm{C}$ isotope fractionation rests heavily, although not exclusively, upon isotope charge exchange (ICE) reactions, such as:

$$
\begin{aligned}
{ }^{13} \mathrm{C}^{+}+\mathrm{CO} & \rightleftharpoons \mathrm{C}^{+}+{ }^{13} \mathrm{CO}+35 \mathrm{~K}, \\
{ }^{13} \mathrm{CO}+\mathrm{HCO}^{+} & \rightleftharpoons \mathrm{CO}+\mathrm{H}^{13} \mathrm{CO}^{+}+17 \mathrm{~K}, \\
{ }^{13} \mathrm{C}^{+}+\mathrm{CN} & \rightleftharpoons \mathrm{C}^{+}+{ }^{13} \mathrm{CN}+31 \mathrm{~K},
\end{aligned}
$$

(Watson et al. 1976; Langer et al. 1978, 1984; Roueff et al. 2015). Since these forward reactions are slightly exothermic, in cold molecular gas these reactions can lead isotope fractionation. However, there are large and complex chemical networks associated with and linking these and other molecules, necessitating sophisticated chemical modelling. There is also competition with selective photo-dissociation (SPD): for CO UV photo-dissociation is a line process (van Dishoeck \& Black 1988), allowing for isotopologue specific self-shielding and thereby fractionating $\mathrm{CO}$ in the opposite direction to ICE. In contrast, CN photo-dissociation is a continuum process (Lavendy et al. 1987).

Langer et al. (1984) presented time dependent chemical models of molecular cloud chemistry for a range of densities $\left(5 \times 10^{2}-1 \times 10^{5} \mathrm{~cm}^{-3}\right)$, temperatures $(6-80 \mathrm{~K})$, metallicities and $\mathrm{C} / \mathrm{O}$ abundances, with the aim of modelling ${ }^{13} \mathrm{C}$ fractionation. They found large variations between species and with conditions, as well as multiple degeneracies. Three distinctly behaved carbon pools emerged: $\mathrm{CO}, \mathrm{HCO}^{+}$, and other carbon bearing species such as $\mathrm{C}^{+}, \mathrm{H}_{2} \mathrm{CO}, \mathrm{HCN}, \mathrm{CS}, \mathrm{CH}$ etc., with each pool broadly following a given trend in fractionation.

Their key findings were the confirmation that a small decrease in the $[\mathrm{CO}] /\left[{ }^{13} \mathrm{CO}\right]$ ratio from $\mathrm{ICE}$ can, due to the high relative abundance of $\mathrm{CO}$, rapidly deplete the pool of available ${ }^{13} \mathrm{C}$, leading to very elevated ratios such as $[\mathrm{HCN}] /\left[\mathrm{H}^{13} \mathrm{CN}\right]$, which attained ratios as high as $6 \times$ that of $[\mathrm{CO}] /\left[{ }^{13} \mathrm{CO}\right] . \mathrm{HCO}^{+}$on the other hand exists in a complex equilibrium with both other pools and can be fractionated in either direction, although to a lesser extent. It is also very susceptible to variations in metallicity and the $\mathrm{C} / \mathrm{O}$ ratio, but was never found to deviate from the $[\mathrm{CO}] /\left[{ }^{13} \mathrm{CO}\right]$ ratio by more than a factor of 1.5. Combining their results, they predicted a bracketing of the true $\left[{ }^{12} \mathrm{C}\right] /\left[{ }^{13} \mathrm{C}\right]$ ratio:

$$
[\mathrm{CO}] /\left[{ }^{13} \mathrm{CO}\right] \leq\left[{ }^{12} \mathrm{C}\right] /\left[{ }^{13} \mathrm{C}\right] \leq[\mathrm{HCN}] /\left[\mathrm{H}^{13} \mathrm{CN}\right]
$$

while $\left[\mathrm{HCO}^{+}\right] /\left[\mathrm{H}^{13} \mathrm{CO}^{+}\right]$is impossible to interpret in isolation, and extremely challenging even when combined with observations of multiple species and their isotopologue ratios.

Observational studies over the subsequent three decades have presented an even more complex picture. Milam et al. (2005) observed $\mathrm{CN}$ and ${ }^{13} \mathrm{CN}$ along lines of sight to dense clouds in the Milky Way, finding no difference in the isotopologue ratios of $\mathrm{CN}, \mathrm{CO}$ and $\mathrm{H}_{2} \mathrm{CO}$, instead finding that all three ratios increase from $\sim 25$ in the Galactic Centre to $~ 130$ at a Galactic radius of $16.4 \mathrm{kpc}$, ostensibly due to the ISM being more evolved (and hence enriched in ${ }^{13} \mathrm{C}$ ) near the Galactic centre.

On the other hand, Sheffer et al. (2007) used HST UV observations to measure $[\mathrm{CO}] /\left[{ }^{13} \mathrm{CO}\right]$ over 25 lines of sight, finding evidence of $\mathrm{CO}$ isotope fractionation in both positive and negative directions, as well as finding that the $[\mathrm{CN}] /\left[{ }^{13} \mathrm{CN}\right]$ ratio appeared to anti-correlate with $[\mathrm{CO}] /\left[{ }^{13} \mathrm{CO}\right]$, as predicted by Langer et al. (1984).

Similarly, Ritchey et al. (2011) found significant evidence for anti-correlated fractionation of $\mathrm{CO}$ and $\mathrm{CN}$ along lines of sight to diffuse molecular clouds, such as $\zeta$ Oph, which presents $[\mathrm{CO}] /\left[{ }^{13} \mathrm{CO}\right]=167 \pm 15$ and $[\mathrm{CN}] /\left[{ }^{13} \mathrm{CN}\right]=47.3_{-4.4}^{+5.5}$ (Lambert et al. 1994; Crane \& Hegyi 1988). Ritchey et al. (2011) identified $\mathrm{CH}^{+}$ as a potential candidate to reliably constrain the true $\left[{ }^{12} \mathrm{C}\right] /\left[{ }^{13} \mathrm{C}\right]$ ratio as it is produced only in non-thermal processes $^{5}$, so is insusceptible to fractionation. Indeed, towards $\zeta$ Oph $\left[\mathrm{CH}^{+}\right] /\left[{ }^{13} \mathrm{CH}^{+}\right]=67.5 \pm 4.5$, c.f. the ISM standard of 68 (Crane et al. 1991; Milam et al. 2005).

The most recent, state-of-the-art chemical modelling study of isotope fractionation was conducted by Roueff et al. (2015), who aimed to refine the understanding of $\left[{ }^{14} \mathrm{~N}\right] /\left[{ }^{15} \mathrm{~N}\right]$ fractionation by cementing the related $\left[{ }^{12} \mathrm{C}\right] /\left[{ }^{13} \mathrm{C}\right]$ fractionation chemistry. Their detailed chemical model included some of the most up-to-date reactions and chemical networks and explored time dependent isotope fractionation in cold $(10 \mathrm{~K})$, dense $\left(2 \times 10^{4}\right.$ and $\left.2 \times 10^{5} \mathrm{~cm}^{-3}\right)$ molecular clouds, with an elemental $\left[{ }^{12} \mathrm{C}\right] /\left[{ }^{13} \mathrm{C}\right]=68$. One of the significant changes is the addition of the reaction:

$$
\mathrm{HNC}+\mathrm{C} \rightarrow \mathrm{HCN}+\mathrm{C},
$$

${ }^{5} \mathrm{CH}^{+}$is formed via the reaction:

$$
\mathrm{C}^{+}+\mathrm{H}_{2} \rightarrow \mathrm{CH}^{+}+\mathrm{H},
$$

which is endothermic with $\Delta \mathrm{E} / k_{B}=4640 \mathrm{~K}$, so requires localised magnetohydrodynamic shocks to form in molecular clouds (Elitzur \& Watson 1978, 1980; Ritchey et al. 2011) 
(Loison et al. 2014), which couples HCN back into the chemical network post-production, allowing it to be fractionated at any time that the ${ }^{13} \mathrm{C}$ pool is depleted. In addition, Roueff et al. (2015) use an updated $\Delta \mathrm{E} / k_{B}$ for Equation 2 of $17 \mathrm{~K}$, c.f. $9 \mathrm{~K}$ in Langer et al. (1984) (Lohr 1998; Mladenović \& Roueff 2014).

The models of Roueff et al. (2015) have particularly interesting results for the isotopologue ratios of $\mathrm{CN}$, $\mathrm{HCN}$ and $\mathrm{HCO}^{+}$. Like Langer et al. (1984), Roueff et al. (2015) found that in steady state $[\mathrm{CO}] /\left[{ }^{13} \mathrm{CO}\right]$ is only very slightly less than $\left[{ }^{12} \mathrm{C}\right] /\left[{ }^{13} \mathrm{C}\right]$, at 67.4 and 68 in the lower and higher density models respectively. $\mathrm{CN}$ fractionates up in the first model, and down in the second. HCN on the other hand is elevated in all of their models, with steady state ratios consistently $2 \sim 3 \times[\mathrm{CO}] /\left[{ }^{13} \mathrm{CO}\right] . \mathrm{HCO}^{+}$tends to equilibrate with $[\mathrm{CO}] /\left[{ }^{13} \mathrm{CO}\right]$ in all of the models. They also found that the $\mathrm{H}_{2}$ ortho-para ratio has very significant effects on some species, such as CN, but not others, such as HCN.

The fundamental conclusion of the observational and theoretical studies is that isotope fractionation is possible, but that it is complex and hard to constrain due to the multitude of variables driving and opposing fractionation. Even state-of-the-art chemical models are limited in usefulness unless they have been run for the specific cloud conditions in question due to the strong sensitivity to temperature, density and metallicity. However, $\mathrm{HCN}$ appears to be reliably and strongly fractionated away from the elemental abundances, while $\mathrm{HCO}^{+}$can be fractionated in either direction and is very sensitive to the evolutionary stage of the molecular cloud.

It is standard practice, when observing $\mathrm{H}^{13} \mathrm{CN}$, to assume a canonical $[\mathrm{HCN}] /\left[\mathrm{H}^{13} \mathrm{CN}\right]$ abundance ratio (e.g., Pirogov et al. 1995; Nakajima et al. 2011; Tunnard et al. 2015). In light of the chemical modelling discussed above this approach appears to risk being significantly flawed: since HCN preferentially traces colder, denser gas, it is especially sensitive to the effects of isotope fractionation. Also, there is a degeneracy between the kinetic temperature and the isotopologue ratio for $\mathrm{HCN} / \mathrm{H}^{13} \mathrm{CN}$ line ratios; assuming an incorrect $[\mathrm{HCN}] /\left[\mathrm{H}^{13} \mathrm{CN}\right]$ runs the risk of significantly biasing derived $\mathrm{HCN}$ column densities and estimates of the local kinetic temperature.

The chemical studies also suggest that any claims of elevated elemental $\left[{ }^{12} \mathrm{C}\right] /\left[{ }^{13} \mathrm{C}\right]$ ratios, especially in extragalactic sources, must be interpreted with caution, and within not only the appropriate physical context, but also the appropriate chemical context (e.g., as in Henkel et al. 2014). There is also the potential for strong optical depth effects (Szücs et al. 2014), although that particular challenge appears intractable with unresolved, single dish extragalactic observations.

\section{2. $N G C 6240$}

NGC 6240 is an early stage major merger galaxy in the constellation of Ophiuchus. Hosting two active supermassive black holes and possessing an infra-red luminosity $L_{8-1000 \mu \mathrm{m}}=10^{11.73} L_{\odot}$ it is a luminous infrared galaxy (LIRG) and a LINER galaxy (low-ionization nuclear emission-line region) (Komossa et al. 2003; Lutz et al. 2003; Sanders et al. 2003; Véron-Cetty \& Véron
2006). Studies by Tacconi et al. (1999) and Tecza et al. (2000) have shown that while star formation is concentrated in two nuclei, the molecular gas is predominantly in a turbulent, thick inter-nuclear disk. They also demonstrated that the extremely luminous infrared $\mathrm{H}_{2}$ $S(0)$ and $S(1)$ emission $\left(L\left(\mathrm{H}_{2}\right) \sim 2 \times 10^{9} L_{\odot}\right)$ is most likely due to slow $\mathrm{C}$ shocks between gas clouds in this dense, turbulent disk; a finding confirmed by the study of the CO spectral line energy distribution (SLED), complete up to and including $J=13-12$, by Meijerink et al. (2013).

As one of the closest LIRGs the molecular emission in NGC 6240 has been extensively studied. The finding of Greve et al. (2009) that the CO SLED required at least a two component fit has been extended upon by $\mathrm{Pa}$ padopoulos et al. (2014), who fitted the CO SLED of CO from $J=1-0$ to $J=13-12$ with an "inside out" decomposition, where the results of $\mathrm{HCN}$ and $\mathrm{HCO}^{+} J=1-0$ to $J=4-3$ SLED fits are used to constrain the high- $J$ $\mathrm{CO}$ lines, and a third phase fitted to the remaining $\mathrm{CO}$ lines. Two of their key findings for NGC 6240 were a very high $\left[{ }^{12} \mathrm{C}\right] /\left[{ }^{13} \mathrm{C}\right]$ ratio of $300-500$ and that most of the $\mathrm{H}_{2}$ gas mass is inconsistent with self-gravitating and photoelectrically heated gas, requiring either cosmic ray or turbulent heating. Indeed, Meijerink et al. (2013) model the CO SLED as being entirely due to shock heated gas. An independent two phase analysis was also conducted by Kamenetzky et al. (2014), who used a nested sampling routine to model only the CO SLED, finding a hot, diffuse phase and a cold, dense phase.

Papadopoulos et al. (2014) argued that the high $\left[{ }^{12} \mathrm{C}\right] /\left[{ }^{13} \mathrm{C}\right]$ (300-500) abundance ratio they found implies the existence of a very unusual environment in NGC 6240. They suggest that it is either a sign of a prolonged period of star formation with a top heavy initial mass function (IMF) or an inflow of unprocessed gas from the outer edges of the galaxy. However, the massive, wide outflow in CO observed by Feruglio et al. (2013) in NGC 6240 suggests that an extensive inflow of gas would be difficult to maintain. That being said, García-Burillo et al. (2014) observed simultaneous inflows and outflows into/from the circumnuclear disk of NGC 1068, so this possibility cannot be entirely ruled out.

As well as presenting a striking CO SLED, NGC 6240 was shown to possess massive $\left(\sim 120 \mathrm{M}_{\odot} \mathrm{yr}^{-1}\right)$ molecular outflows at $-600,+800 \mathrm{~km} \mathrm{~s}^{-1}$ by Feruglio et al. (2013) in $\mathrm{CO}(1-0)$, while Wang et al. (2014) found that the extended $(5 \mathrm{kpc})$ hard X-ray spectrum $(\sim 6 \mathrm{keV})$ is due to $\sim 2200 \mathrm{~km} \mathrm{~s}^{-1}$ outflows, which they ascribe to the nuclear starburst with a supernova rate of $\sim 2 \mathrm{yr}^{-1}$.

This combination of extreme conditions make NGC 6240 a unique laboratory for star formation physics under unusual conditions.

This paper is outlined as follows: we describe our observations and their analysis in Section 2 and the line extraction in Section 3. We introduce our Monte Carlo Markov Chain - Large Velocity Gradient (MCMC LVG) model and results in Section 4. The results are discussed in Section 5 and we outline our conclusions in Section 6 . We include further details of our model in the Appendix. We adopt cosmological parameters $h=0.70, \Omega_{m}=0.3$ and $\Omega_{\Lambda}=0.7$, giving a luminosity distance of $107 \mathrm{Mpc}$ and 
an angular scale of $0.494 \mathrm{kpc} /{ }^{\prime \prime}$ at $z=0.0245$ (Wright 2006; Papadopoulos et al. 2014). To avoid confusion, we note that throughout this work, unless explicitly stated otherwise, we present results as $x_{b}^{a}$, where $a$ and $b$ denote the values at the upper and lower limits on the $68 \%$ credible interval.

\section{OBSERVATIONS}

We obtained observations of $\mathrm{H}^{13} \mathrm{CN}(1-0), \mathrm{H}^{13} \mathrm{CO}^{+}(1-$ $0)$ and $\mathrm{SiO}(2-1)$ in NGC 6240 with the Plateau de Bure Interferometer (PdBI) on 2008 February 21 and 2008 March 7 in the most extended configuration (PI: J. Graciá-Carpio), obtaining minimum and maximum baselines of $59.7 \mathrm{~m}(16.8 \mathrm{k} \lambda)$ and $760 \mathrm{~m}(215.8 \mathrm{k} \lambda)$ respectively, centred on the sky frequency of $84.6 \mathrm{GHz}$. Using natural weighting to maximise sensitivity we obtained a synthesised beam $1.86^{\prime \prime} \times 0.74^{\prime \prime}$.

The data were reduced in CLIC, part of the IRAM software package GILDAS ${ }^{6}$, while for the UV analysis and imaging we used the GILDAS program, MAPPING.

\section{SPECTRUM EXTRACTION AND DATA ANALYSIS}

Due to the poor signal to noise of the UV visibilities we analysed the data independently in both the UV plane and in the final imaged datacube. These two methods are outlined below, and produced spectra consistent on a channel by channel basis, within the channel uncertainties.

Elliptical Gaussian and disk models were fitted to the UV visibilities on a channel-by-channel basis, using the GILDAS task UV_FIT and $30 \mathrm{MHz}$ channels. The fitted fluxes and fit rms's were identical for the two models and we adopted the elliptical Gaussian model. Fitting of channels narrower than $30 \mathrm{MHz}$ was attempted but produced pathological fits in multiple channels due to the poor $\mathrm{S} / \mathrm{N}$ of the visibilities. The spectrum obtained is shown in Figure 1, where we fit two Gaussian profiles and a $0^{\text {th }}$ order polynomial baseline $e^{7}$. The $\mathrm{SiO}(2-1)$ line is clearly detected and there is a possible detection of $\mathrm{H}^{13} \mathrm{CN}(1-0)$. The significance of this detection is quantified in Section 3.2 below. Channel errors are shown at the $\pm 1 \sigma$ level and are unique for each channel, based on uncertainty in the fitted flux. The median channel error is $\pm 0.8 \mathrm{mJy}$.

A concern with the UV fit to each channel is the possibility of fitting to a different spatial centre, and thereby unintentionally applying a spatial pseudoaveraging across the channels. The majority of the channels fitted a centre consistent within the uncertainties $\left(\sim \pm 0.06^{\prime \prime}\right)$, while the full range is $0.3^{\prime \prime}$ in Dec and $0.1^{\prime \prime}$ in RA, dominated by four outlying channels. These offsets are $<25 \%$ of the fitted FWHMs, and $<5 \%$ of the aperture used for extracting the spectrum from the imaged spectrum (see below), and so this is not a significant effect here.

\footnotetext{
${ }^{6}$ http://www.iram.fr/IRAMFR/GILDAS/.

7 All reasonable fits with a $1^{\text {st }}$ order polynomial baseline had a gradient of almost zero. However, due to the randomised starting conditions we use to check for convergence, some solutions with a $1^{\text {st }}$ order polynomial were pathological, and we adopt instead the stable $0^{\text {th }}$ order.
}

For imaging, we first continuum subtracted the data by extracting the visibilities from the $210 \mathrm{MHz}$ line free region from $86.47 \mathrm{GHz}$ to $86.67 \mathrm{GHz}$ (rest frame) to define the continuum, channel averaging the visibilities, and then subtracting the channel averaged visibilities in the UV plane. The visibilities were imaged in MAPPING and the spectrum extracted in CASA (McMullin et al. 2007 ) with an elliptical aperture $5.58^{\prime \prime} \times 2.22^{\prime \prime}, \mathrm{PA}=162^{\circ}$ (three times the clean beam), centred on the phase centre $\mathrm{RA}=16^{\mathrm{h}} 52^{\mathrm{m}} 58^{\mathrm{s}} .890$, Dec $=+02^{\circ} 24^{\prime} 03^{\prime \prime} .90$. This includes conversions from mJy beam ${ }^{-1}$ to mJy and allows for a slightly extended source.

The noise in the imaged cube was estimated with the GILDAS task NOISE to be $0.2 \mathrm{mJy}_{\text {beam }}{ }^{-1}$. To estimate the noise in the spectrum we placed eight apertures, identical to that used for the spectrum extraction, arranged around the science aperture so as to create a regular $3 \times 3$ grid with the science spectrum in the centre. We then extracted the spectra from within these eight apertures. Since the cube is continuum subtracted, the signal in these apertures should be entirely noise (both thermal, and artificial from processing). For each spectrum the rms about zero was found, and then these eight rms's were averaged to produce a final rms for the imaged spectrum of $0.6 \mathrm{mJy}$ channel $^{-1}$. The spectrum is shown in Figure 2. We fitted a single Gaussian to the $\mathrm{SiO}(2-1)$ line and find a reduced $\chi^{2}$ of 2.12. Attempts to fit a second Gaussian component consistently lead to pathological results due to all of the putative $\mathrm{H}^{13} \mathrm{CN}(1-0)$ flux lying in a single channel.

The UV fitted and imaged spectra are compared in Figure 3 . The channel errors have been offset horizontally for clarity. Every channel is consistent between the two spectra within $1 \sigma$.

\subsection{Continuum}

We used the $210 \mathrm{MHz}$ region from $86.47 \mathrm{GHz}$ to $86.67 \mathrm{GHz}$ (rest frame) to define the continuum. This region, expected to be line free, was verified as such by an inspection of the UV fitted spectrum. The imaged continuum is shown in Figure 4 and we find an aperture extracted flux density of $11.3 \pm 1.7 \mathrm{mJy}$ (using the same aperture and method as for the spectrum extraction). This is noticeably larger than the flux density of the elliptical Gaussian fitted to the continuum UV visibilities, which gives a flux density of $9.70 \pm 0.29 \mathrm{mJy}(9.7 \pm 1.5 \mathrm{mJy}$ including the $15 \%$ absolute flux calibration uncertainty), although it is still consistent within $1 \sigma$. This is in part due to the extended irregularly shaped emission to the south-east of the pointing centre, which is not accounted for in the single component UV fit. Ideally we would fit further components to the residual visibilities (see e.g., Feruglio et al. 2013), but the poor signal to noise ratio of our data prevents meaningfully fitting additional components.

An estimate of the continuum was also provided by the baseline fitted to the UV fitted spectrum. This measured a continuum flux density of $9.58 \pm 0.19 \mathrm{mJy}$, or, including our $15 \%$ absolute flux calibration uncertainty, $9.6 \pm 1.5 \mathrm{mJy}$.

Our continuum flux density is slightly than expected: Nakanishi et al. (2005) found $16.6 \pm 1.7 \mathrm{mJy}$ at 


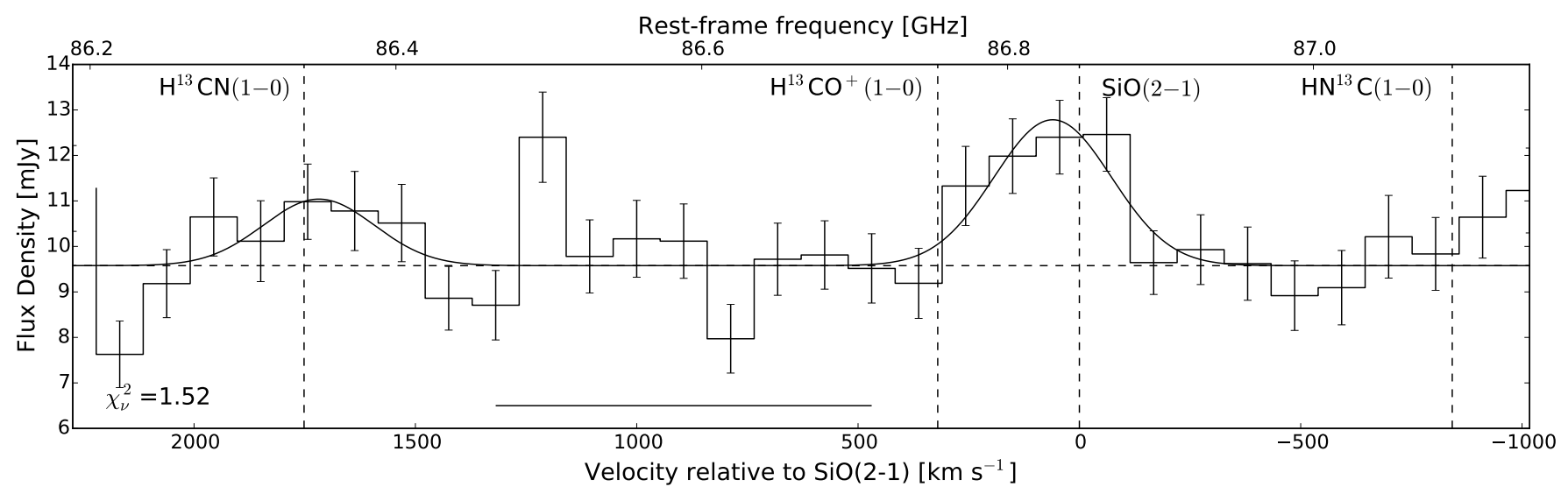

FIG. 1. - The UV fitted spectrum and the least squares two Gaussian plus baseline fit. Velocities are relative to the $\mathrm{SiO}(2-1)$ line centre, and rest frame frequencies in $\mathrm{GHz}$ are given at the top of the plot. The $1 \sigma$ channel uncertainties (median $\pm 0.8 \mathrm{mJy}$ ) from the UV fitting are shown, and do not include the absolute flux calibration uncertainty. The horizontal bar indicates the region identified as line free and used for the continuum subtraction in the imaging step. The reduced $\chi^{2}$ of the fit is 1.52 .

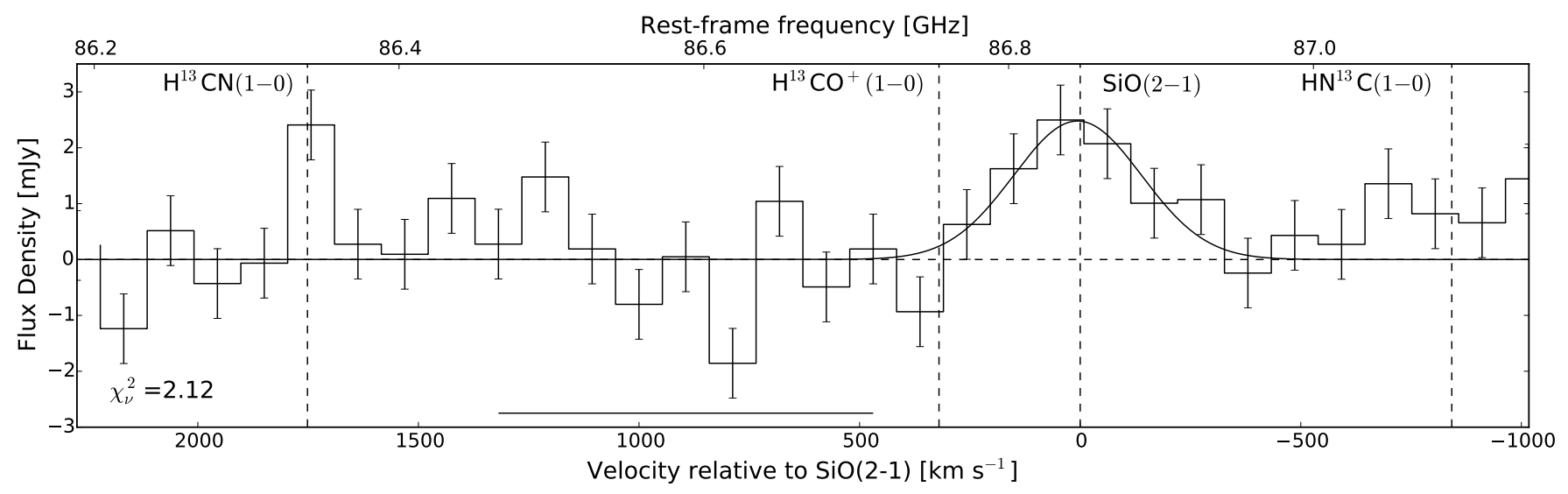

FIG. 2. - The imaged spectrum, extracted with an aperture as described in Section 3, and the least squares Gaussian fit to the $\mathrm{SiO}(2-1)$ line. Velocities are relative to the $\mathrm{SiO}(2-1)$ line centre, and rest frame frequencies in $\mathrm{GHz}$ are given at the top of the plot. The $1 \sigma$ rms channel uncertainties $( \pm 0.6 \mathrm{mJy})$ are shown, and do not include the absolute flux calibration uncertainty. The horizontal bar indicates the region used for the continuum. The reduced $\chi^{2}$ of the fit is 2.12 .

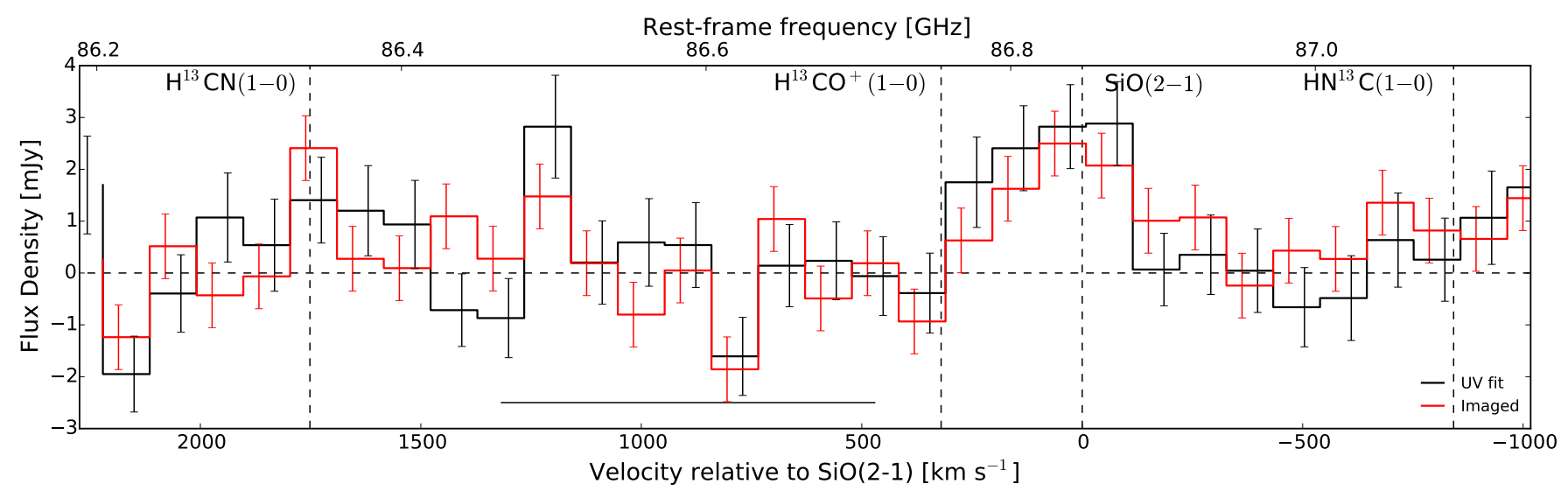

FIG. 3.- A comparison of the UV fitted and Imaged spectra. Axes are as in Figures 1 and 2. The UV fitted spectrum has been continuum subtracted using the baseline fitted simultaneously with the Gaussian lines. Channel uncertainties have been offset left and right for clarity. 


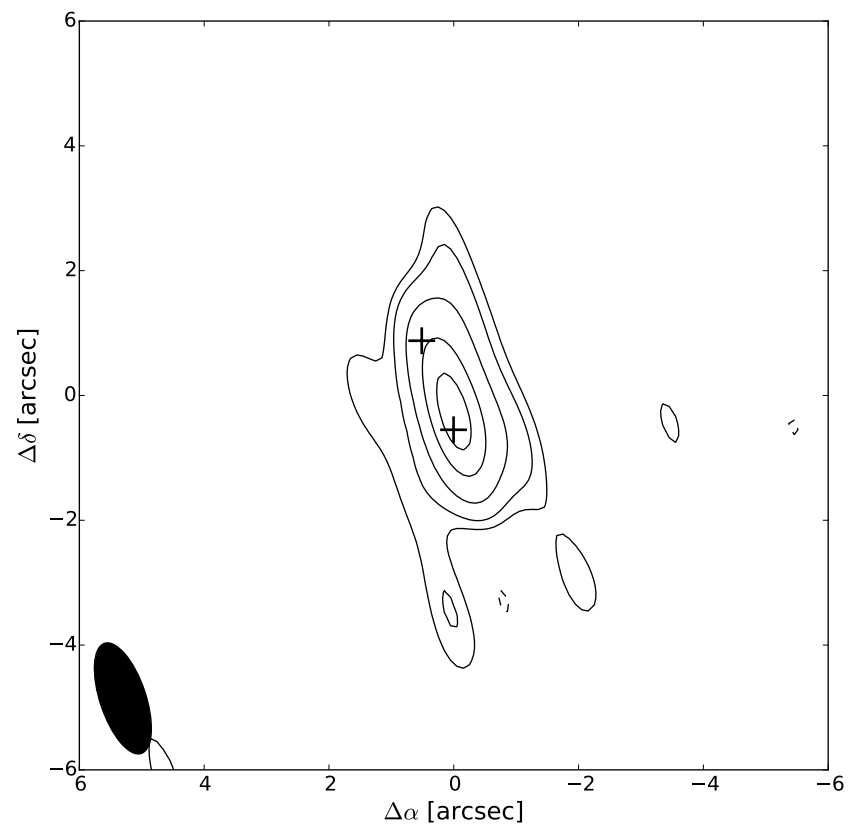

FIG. 4.- The NGC $62403 \mathrm{~mm}$ continuum. Black crosses mark the positions of the AGNs as reported in Hagiwara et al. (2011). Contours are at $-3,3,5,10,20$ and $30 \sigma$, where $\sigma=1.63 \times$ $10^{-4} \mathrm{Jy} \mathrm{beam}^{-1}$.

$\sim 88.6 \mathrm{GHz}$ rest frame and $10.8 \pm 1.1 \mathrm{mJy}$ at $\sim 110.2 \mathrm{GHz}$ rest frame, with the Nobeyama Millimeter Array and RAINBOW interferometers. Their measurement is consistent with our imaged continuum at the $2 \sigma$ level. Expanding the aperture used for the imaged continuum does not significantly change the recovered flux density.

\subsection{Spectral Lines}

Given the low signal to noise ratio of our observations we adopt an elementary statistical approach to line identification. A priori, we expect to detect $\mathrm{H}^{13} \mathrm{CN}(1-0)$ and $\mathrm{H}^{13} \mathrm{CO}^{+}(1-0)$ based on previous detections of $\mathrm{HCN}$ and $\mathrm{HCO}^{+}$in NGC 6240, while we expect to detect $\mathrm{SiO}(2-1)$ based on comparisons with other LIRGs and major mergers. We give parameters for these lines in Table 1.

Only $\mathrm{SiO}(2-1)$ is clearly detected in both the UV fitted and imaged spectra. The $\mathrm{H}^{13} \mathrm{CN}(1-0)$ line appears to be present in the UV fitted spectrum, but not clearly so in the imaged spectrum, and there is no sign of $\mathrm{H}^{13} \mathrm{CO}^{+}(1-0)$ in either. While, if detected, it would be at least partially blended with the $\mathrm{SiO}(2-1)$ line, there is absolutely no sign of emission redwards of the $\mathrm{H}^{13} \mathrm{CO}^{+}(1-0)$ line centre, or bluewards in the residuals of the $\mathrm{SiO}(2-1)$ line fit.

For our statistical test of the line detections we treat the channel uncertainties as Gaussian. We sum the channels within $\pm 400 \mathrm{~km} \mathrm{~s}^{-1}$ of the line centre and sum the channel rms's in quadrature, to create a single 'detection' channel and quantify this signal in units of $\sigma_{\text {detect }}$, the rms of the detection channel. We define our upper limit on the flux density, $S_{\max }$, as the flux density which has a $2 \%$ chance of being measured at the value we see or less if the line is in fact present, i.e.

$$
S_{\max }=\left[S_{\text {detect }}+\sqrt{2 \sigma^{2}} \operatorname{erf}^{-1}(2 \mathrm{p}-1)\right],
$$

where $S_{\text {detect }}$ is the measured signal in the detection channel, p is our 0.02 probability and erf is the Gauss error function. For $\mathrm{p}=0.02$ this gives $S_{\max }=S_{\text {detect }}+$ $2.054 \sigma\left(\right.$ Masci 2011) ${ }^{8}$.

TABLE 1

Observed Line Parameters

\begin{tabular}{lcccc}
\hline Line & $\begin{array}{c}\nu_{0} \\
{[\mathrm{GHz}]}\end{array}$ & $\begin{array}{c}\mathrm{E}_{\mathrm{u}} / \mathrm{k} \\
{[\mathrm{K}]}\end{array}$ & $\begin{array}{c}S_{\text {line }}(\mathrm{UV}) \\
{\left[\mathrm{Jy} \mathrm{km} \mathrm{s}^{-1}\right]}\end{array}$ & $\begin{array}{c}S_{\text {line }}(\text { imaged }) \\
{\left[\mathrm{Jy} \mathrm{km} \mathrm{s}^{-1}\right]}\end{array}$ \\
\hline \hline $\mathrm{H}^{13} \mathrm{CN}(1-0) .^{\mathrm{a}}$ & 86.340 & 4.14 & $0.46 \pm 0.07$ & $<0.8$ \\
$\mathrm{H}^{13} \mathrm{CO}^{+}(1-0)^{\mathrm{a}}$ & 86.754 & 4.16 & $<0.4$ & $<0.4$ \\
$\mathrm{SiO}(2-1) \ldots{ }^{\mathrm{b}}$ & 86.847 & 6.25 & $1.10 \pm 0.15$ & $0.90 \pm 0.09$ \\
\hline
\end{tabular}

The uncertainties quoted here do not include the $15 \%$ absolute calibration uncertainty.

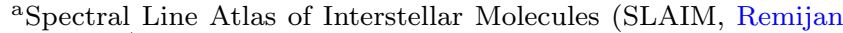
et al. 2007).

${ }^{\mathrm{b}}$ The Cologne Database for Molecular Spectroscopy (CDMS, Müller et al. 2005).

We use two measures to test whether the line is actually detected. Firstly, we test against the null hypothesis that we see a signal at least as bright as in the data, given that there is no line present - i.e., we assume that there is no chance of absorption. This is a one-tailed test with probability

$$
\alpha=\frac{1}{2}\left[1-\operatorname{erf}\left(\frac{x-\mu}{\sqrt{2 \sigma^{2}}}\right)\right],
$$

and a value consistent with no line is 0.5 .

Secondly, we test against the null hypothesis that we see a signal with an absolute value at least as great as is seen, given that there is no line present - i.e, we allow for absorption. This is a two-tailed test with probability

$$
\alpha=1-\operatorname{erf}\left(\frac{|x-\mu|}{\sqrt{2 \sigma^{2}}}\right),
$$

and a value consistent with no line is 1 . While the onetailed test specifically searches for emission lines, the twotailed test makes no such distinction.

For the aperture extracted $\operatorname{SiO}(2-1)$ line and continuum region we found one-tailed values of $7.0 \times 10^{-7}$ and 0.53 respectively, precisely as expected for a clear line detection and a flat continuum. The two-tailed values are $1.4 \times 10^{-6}$ and 0.95 , again consistent with a clear line detection and a flat continuum.

For the imaged $\mathrm{H}^{13} \mathrm{CN}(1-0)$ line the one and twotailed tests gave 0.014 and 0.028 respectively, while the UV fitted line gave 0.014 and 0.004. While not entirely conclusive, these tests argue for a detection of the $\mathrm{H}^{13} \mathrm{CN}(1-0)$ line in the UV fitted spectrum. For $\mathrm{H}^{13} \mathrm{CO}^{+}(1-0)$ we subtracted the $\mathrm{SiO}(2-1)$ line fit from the spectrum before running the tests. In all cases we find values completely consistent with no line detection with one and two-tailed tests giving 0.51 and 0.98 respectively.

For the $\mathrm{H}^{13} \mathrm{CN}(1-0)$ line we find an upper limit $S_{\nu} \mathrm{d} v<0.8 \mathrm{Jy} \mathrm{kms}^{-1}$ from the imaged spectrum and $S_{\nu} \mathrm{d} v<0.9 \mathrm{Jy} \mathrm{km} \mathrm{s}^{-1}$ from the UV fitting. The imaged spectrum cannot be well fitted with a Gaussian

8 wise2.ipac.caltech.edu/staff/fmasci/UpperLimits_FM2011.pdf 
due to almost all of the flux residing in one channel, but for the UV fitted spectrum the Gaussian fit to the line gives $S_{\nu} \mathrm{d} v=0.46 \pm 0.07 \mathrm{Jy} \mathrm{km} \mathrm{s}^{-1}$. The $\mathrm{SiO}(2-1)$ line was clearly detected in both spectra, and was fitted with line fluxes of $S_{\nu} \mathrm{d} v=1.10 \pm 0.15 \mathrm{Jy} \mathrm{km} \mathrm{s}^{-1}$ and $S_{\nu} \mathrm{d} v=0.90 \pm 0.09 \mathrm{Jy} \mathrm{km} \mathrm{s}^{-1}$ in the UV fitted and imaged spectra respectively (not including the $15 \%$ absolute calibration uncertainty). For $\mathrm{H}^{13} \mathrm{CO}^{+}(1-0)$ we first subtracted the $\mathrm{SiO}(2-1)$ Gaussian line fit before summing the channel fluxes. We obtain an upper limit of $S_{\nu} \mathrm{d} v<0.4 \mathrm{Jy} \mathrm{km} \mathrm{s}^{-1}$ for both spectra. Attempts to simultaneously fit two Gaussians to the $\mathrm{SiO}(2-1)$ and $\mathrm{H}^{13} \mathrm{CO}^{+}(1-0)$ line centres consistently reported zero line flux for $\mathrm{H}^{13} \mathrm{CO}^{+}(1-0)$, consistent with a nondetection/upper limit. The measured fluxes and upper limits are recorded in Table 1.

We present $30 \mathrm{MHz}$ channels maps for $\mathrm{H}^{13} \mathrm{CN}(1-0)$, $\mathrm{H}^{13} \mathrm{CO}^{+}(1-0)$ and $\mathrm{SiO}(2-1)$ in Figure $5 . \mathrm{H}^{13} \mathrm{CN}(1-0)$ is seen only in the channel containing the line centre, at a peak level of $3 \sigma$. It is concentrated between the two nuclei, as was seen for $\mathrm{HCN}(1-0)$ by Nakanishi et al. (2005). There is no sign of $\mathrm{H}^{13} \mathrm{CO}^{+}(1-0)$ in any of the channels, consistent with the non-detection in the UV fitted and imaged spectra. Finally, $\mathrm{SiO}(2-1)$ appears at the 3 and $4 \sigma$ level in the 0 and $-110 \mathrm{~km} \mathrm{~s}^{-1}$ channels centred on the southern nucleus, with a possible $3 \sigma$ detection in the $+110 \mathrm{~km} \mathrm{~s}^{-1}$ channel about the northern nucleus.

We briefly note than there is no sign of $\mathrm{HN}^{13} \mathrm{C}(1-0)$ in either of the spectra. Also, if the line were detected it would extend beyond the edge of our spectrum, hindering its analysis. We mark the line frequency in Figures 1 and 2 for reference.

\subsection{Spatial Filtering and Missing Flux}

Interferometers sample discrete spatial frequencies, and as such may underestimate the true flux of a source. As there are no single dish observations of any of our targeted lines we have no direct means of measuring the fraction of recovered flux and instead use comparisons with other line and continuum measurements from the literature. Nominally, our observations should only resolve out emission on scales larger than $12^{\prime \prime}$, three times larger than the largest line and continuum structures seen by Nakanishi et al. (2005), Iono et al. (2007) and Scoville et al. (2015), so it is unlikely that we have any significant loss of flux. However, given the detection of $\mathrm{CO}$ on scales $\sim 25^{\prime \prime}$ by Feruglio et al. (2013) we proceed with caution, despite not expecting $\mathrm{HCN}$ or $\mathrm{HCO}^{+}$emission to be similarly extended.

Nakanishi et al. (2005) found the $\mathrm{HCN}(1-0)$ line to be more compact than both the continuum and $\mathrm{HCO}^{+}(1-$ $0)$. The $\mathrm{HCO}^{+}(1-0)$ line was slightly more extended, but still only out to scales $\sim 4^{\prime \prime}$. It is of course possible that the observations of Nakanishi et al. (2005) are also filtering a significant fraction of the flux, and indeed their single dish flux to interferometric flux ratio was 0.68 for $\mathrm{HCN}(1-0)$, using the single dish data of Solomon et al. (1992). However, the single dish fluxes are extremely variable, and comparisons with more recent single dish observations give flux ratios of $1.1 \pm 0.4$ (Greve et al. 2009) and $0.9 \pm 0.4$ (Krips et al. 2008).

The high spatial resolution $\left(0.5^{\prime \prime}\right)$ interferometric
(ALMA) HCN(4-3) observations of Scoville et al. (2015) have APEX single dish counterparts from Papadopoulos et al. (2014) and a flux ratio $0.90 \pm 0.16$, i.e., consistent with no spatial filtering/almost complete flux recovery. While extrapolating from the $J=4-3$ transition to our $J=1-0$ transitions is unacceptably unreliable, combined with the very compact $\mathrm{HCN}(1-0)$ emission and the comparisons in the previous paragraph this comparison does argue for our observations recovering most of the $\mathrm{H}^{13} \mathrm{CN}(1-0)$ flux.

We note that when comparing ALMA observations with JCMT fluxes Scoville et al. (2015) raised significant concerns regarding the calibration accuracy of the single dish measurements.

Wang et al. (2013) observed $\mathrm{SiO}(2-1)$ in a six local galaxies with the IRAM 30m, including NGC 6240. They found a double peaked line profile, with a combined flux of $0.72 \pm 0.30 \mathrm{Jy} \mathrm{km} \mathrm{s}^{-19}$, consistent with our observations. Wang et al. (2013) also observe what may be a marginal $\mathrm{H}^{13} \mathrm{CO}^{+}$detection, with a peak $T_{A}^{*} \simeq 0.55 \mathrm{mK}$. We approximate this as corresponding to $\simeq 0.08 \mathrm{Jy} \mathrm{km} \mathrm{s}^{-1}$, consistent with our upper limit on the line of $0.4 \mathrm{Jy} \mathrm{km} \mathrm{s}^{-1}$.

\subsection{The LTE limit}

Papadopoulos et al. (2014) reported the discovery of an extremely high $\left[{ }^{12} \mathrm{C}\right] /\left[{ }^{13} \mathrm{C}\right]$ abundance ratio in NGC 6240, evidenced by the $[\mathrm{CO}] /\left[{ }^{13} \mathrm{CO}\right]$ ratio of $300-500$. Here we test whether this ratio is compatible with our observed $\mathrm{H}^{13} \mathrm{CN}(1-0)$ line flux under the assumption of local thermodynamic equilibrium (LTE).

We compare our $\mathrm{H}^{13} \mathrm{CN}(1-0)$ line flux to an aggregate of literature measurements of the $\operatorname{HCN}(1-0)$ line using

$$
\begin{aligned}
S_{\nu} & =\frac{2 k T_{A} \nu^{2}}{c^{2}} \Omega_{A}, \\
T_{A} \mathrm{~d} v & =\frac{h c^{3}}{8 \pi k \nu^{2}} N_{u} A_{u l}\left(\frac{\Omega_{s}}{\Omega_{A}}\right)\left(\frac{1-e^{-\tau}}{\tau}\right),
\end{aligned}
$$

where $\Omega_{A, S}$ is the solid angle of the primary beam and source respectively, $N_{u}$ is the column density of the species in level $u$ in $\mathrm{cm}^{-2}$ and $A_{u l}$ is the Einstein A coefficient for the transition between levels $u$ and $l$ (Goldsmith \& Langer 1999). Combining these equations and assuming that $\mathrm{HCN}$ and $\mathrm{H}^{13} \mathrm{CN}$ share the same excitation temperature, $T_{\mathrm{ex}}$, so that $N_{\mathrm{HCN}, J} / N_{\mathrm{H}^{13} \mathrm{CN}, J}=$ $[\mathrm{HCN}] /\left[\mathrm{H}^{13} \mathrm{CN}\right]$, we obtain for the $J=1-0$ transition:

$$
\frac{S_{1} \mathrm{~d} v_{1}}{S_{2} \mathrm{~d} v_{2}}=\frac{[\mathrm{HCN}]}{\left[\mathrm{H}^{13} \mathrm{CN}\right]} \frac{A_{1,1-0}}{A_{2,1-0}} \frac{\tau_{2}\left(1-e^{-\tau_{1}}\right)}{\tau_{1}\left(1-e^{-\tau_{2}}\right)},
$$

where the subscripts 1 and 2 refer to $\mathrm{HCN}$ and $\mathrm{H}^{13} \mathrm{CN}$ respectively, Therefore, the lower limit on $[\mathrm{HCN}] /\left[\mathrm{H}^{13} \mathrm{CN}\right]$ for a given flux ratio is found when both lines are optically thin ${ }^{10}$. In this optically thin LTE case and using

9 Converted from the $T_{A}^{*}$ fluxes in their Table 1, using their quoted $F_{\text {eff }}=95 \%$ and the $30 \mathrm{~m}$ conversion factor $S / T_{A}^{*}=$ $3.06 F_{e f f} / A_{e f f} \mathrm{Jy} \mathrm{K}{ }^{-1}$, with $A_{e f f}=0.63$ from http://www.iram. es/IRAMES/mainWiki/Iram30mEfficiencies.

10 Assuming that the optical depth of $\operatorname{HCN}(1-0)$ is always greater than that of $\mathrm{H}^{13} \mathrm{CN}(1-0)$ - a safe assumption. 

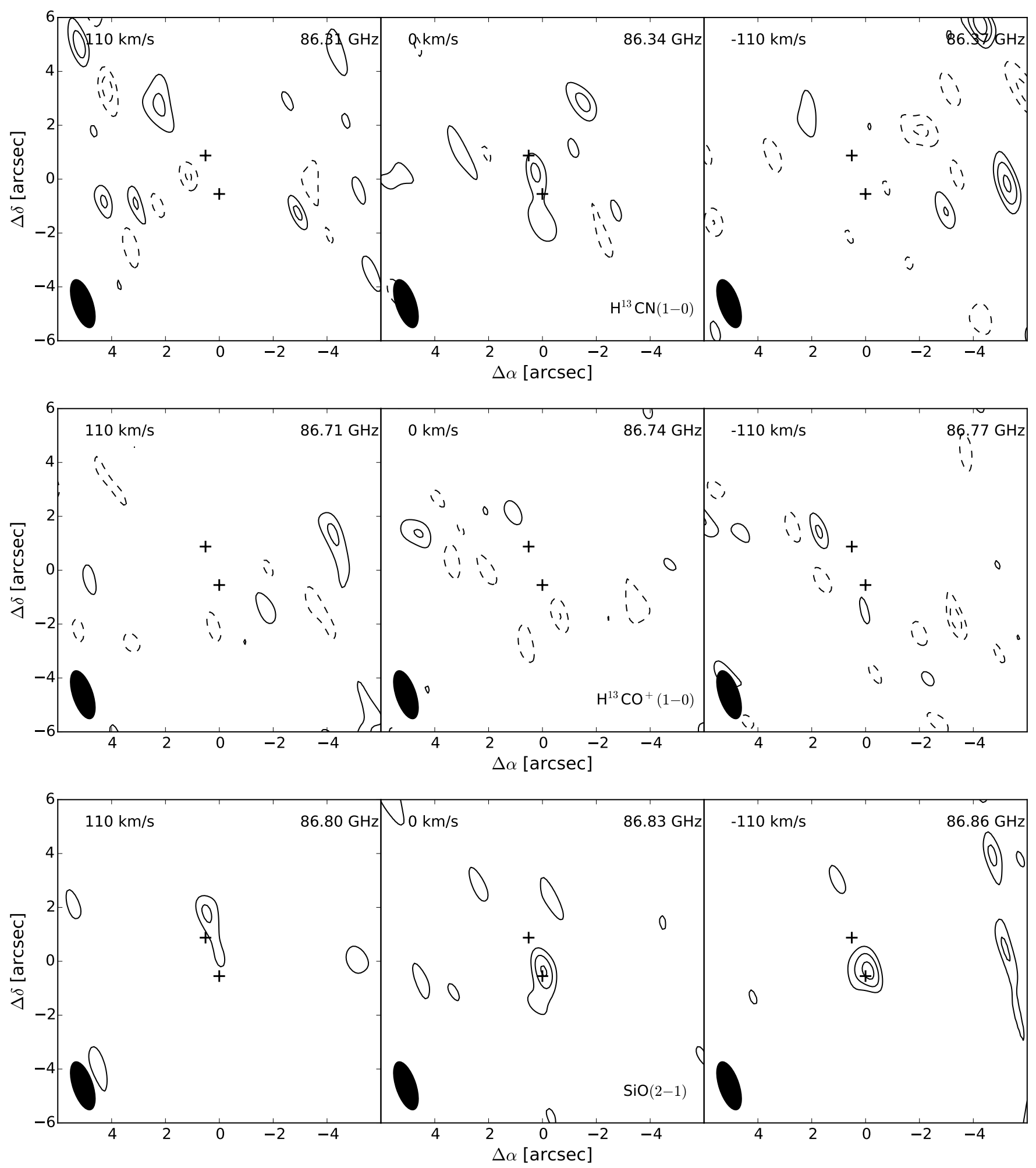

FIG. 5.- Continuum subtracted channel maps for $\mathrm{H}^{13} \mathrm{CN}(1-0), \mathrm{H}^{13} \mathrm{CO}^{+}(1-0)$ and $\mathrm{SiO}(2-1)$. Black crosses mark the AGN as reported in Hagiwara et al. (2011) Contours are at $-3,-2,2,3$ and $4 \sigma$, where $\sigma=3.9 \times 10^{-4} \mathrm{Jy}_{\text {beam }}{ }^{-1}$. $\mathrm{H}^{13} \mathrm{CN}(1-0)$ appears to be located between the two nuclei, co-located with the the $\operatorname{HCN}(1-0)$ (Nakanishi et al. 2005). $\operatorname{SiO}(2-1)$ on the other hand appears to be concentrated around the more active southern nucleus. 
the upper limit on our $\mathrm{H}^{13} \mathrm{CN}(1-0)$ line flux, we find $[\mathrm{HCN}] /\left[\mathrm{H}^{13} \mathrm{CN}\right] \geq 12$. However, exploring a range of optical depths we find that constrained by the $J=1-0$ lines alone the $[\mathrm{HCN}] /\left[\mathrm{H}^{13} \mathrm{CN}\right]$ could be as large as 1000 , consistent with the high values found by Papadopoulos et al. (2014). We further constrain the isotopologue ratio in Section 4 using LVG modelling.

A high $\left[{ }^{12} \mathrm{C}\right] /\left[{ }^{13} \mathrm{C}\right]$ ratio, leading to high $[\mathrm{HCN}] /\left[\mathrm{H}^{13} \mathrm{CN}\right]$ and $\left[\mathrm{HCO}^{+}\right] /\left[\mathrm{H}^{13} \mathrm{CO}^{+}\right]$ratios, is also consistent with $\mathrm{SiO}(2-1)$ being brighter than both $\mathrm{H}^{13} \mathrm{CN}(1-0)$ and $\mathrm{H}^{13} \mathrm{CO}^{+}(1-0)\left(\mathrm{SiO}(2-1) / \mathrm{H}^{13} \mathrm{CO}^{+}(1-0)>3\right)$; $\mathrm{SiO}(2-1)$ is not over-luminous, rather the low abundances of the ${ }^{13} \mathrm{C}$ isotopologues leads to unusually faint $\mathrm{H}^{13} \mathrm{CN}(1-0)$ and $\mathrm{H}^{13} \mathrm{CO}^{+}(1-0)$ lines. Usero et al. (2004) found $\mathrm{SiO}(2-1) / \mathrm{H}^{13} \mathrm{CO}^{+}(1-0)$ intensity ratios of about 3 in some regions of NGC 1068, but this galaxy is unusually bright in $\mathrm{SiO}$.

\section{LVG MODELLING}

We use the publicly available code RADEX ${ }^{11}$ (van der Tak et al. 2007) with collisional data from the Leiden LAMBDA database (Green \& Thaddeus 1974; Flower 1999; Dayou \& Balança 2006; Lique et al. 2006; Dumouchel et al. 2010; Yang et al. 2010) to model spectral line ratios as a function of the gas phase conditions. We employ an MCMC script using the Metropolis-Hastings algorithm (Metropolis et al. 1953; Hastings 1970) to explore the parameter space. Details of our MCMC LVG model are included in Appendix A. We use this code to fit three gas phases and six species simultaneously.

We combine our PdBI data with literature data for $\mathrm{HCN}$ and $\mathrm{HCO}^{+}$shown in Table 2 and adopt $\mathrm{CO}$ and ${ }^{13} \mathrm{CO}$ data from Papadopoulos et al. (2014). We have available to us the complete $\mathrm{HCN}$ and $\mathrm{HCO}^{+}$ladders from $J=1-0$ to $J=4-3$ except for $\operatorname{HCO}^{+}(2-1)$, the complete CO SLED up to and including $J=13-12$, and the ${ }^{13} \mathrm{CO} J=1-0,2-1$ and $6-5$ lines.

In collecting data from a range of telescopes and over a range of frequencies it is essential to consider whether there is a common putative source size and whether the single dish beam coupling to the source varies significantly between observations. We first convert the line fluxes from our observations and the literature into line luminosities:

$L^{\prime}=3.25 \times 10^{7}\left(\frac{\nu_{\mathrm{obs}}}{\mathrm{GHz}}\right)^{-2}\left(\frac{D_{\mathrm{L}}}{\mathrm{Mpc}}\right)^{2}(1+z)^{-3}\left(\frac{\int_{\Delta v} S_{v} \mathrm{~d} v}{\mathrm{Jy} \mathrm{km} \mathrm{s}^{-1}}\right)$,

where $D_{\mathrm{L}}$ is the luminosity distance of the source and $L^{\prime}$ has units of $\mathrm{K} \mathrm{km} \mathrm{s}^{-1} \mathrm{pc}^{2}$ (Solomon et al. 1992). Ideally we would have a complete, spatially resolved sample of lines so that we could precisely compare the line brightnesses at specific positions in the source, ensuring that we were comparing cospatial regions of equal size. Working instead with almost entirely unresolved single dish observations we make the following approximations.

Beam coupling: Interferometric observations of the dense gas tracers exist for only the $J=1-0$ and

11 http://home.strw.leidenuniv.nl/ moldata/radex.html
TABLE 2

LVG Line InPUTS

\begin{tabular}{lccc}
\hline Line & $\begin{array}{c}\nu_{0} \\
{[\mathrm{GHz}]}\end{array}$ & $\begin{array}{c}S_{\text {line }} \\
{\left[\mathrm{Jy} \mathrm{km} \mathrm{s}^{-1}\right]}\end{array}$ & References $^{\mathrm{a}}$ \\
\hline \hline $\mathrm{HCN}(1-0)$ & 88.63 & $14 \pm 2$ & $1,2,3,4$ \\
$\mathrm{HCN}(2-1)$ & 177.3 & $44 \pm 7$ & 3 \\
$\mathrm{HCN}(3-2)$ & 265.9 & $74 \pm 7$ & $3,4,5$ \\
$\mathrm{HCN}(4-3)$ & 354.5 & $41 \pm 6$ & 5,6 \\
$\mathrm{H}^{13} \mathrm{CN}(1-0)$ & 86.34 & $0.46 \pm 0.07$ & $\mathrm{x}$ \\
$\mathrm{HCO}^{+}(1-0)$ & 89.19 & $21 \pm 3$ & 2 \\
$\mathrm{H}^{13} \mathrm{CO}^{+}(1-0)$ & 86.75 & $<0.44$ & $\mathrm{x}$ \\
$\mathrm{HCO}^{+}(3-2)$ & 267.6 & $141 \pm 21$ & 5 \\
$\mathrm{HCO}^{+}(4-3)$ & 356.7 & $74 \pm 9$ & 2,5 \\
\hline
\end{tabular}

${ }^{\mathrm{a}} 1=$ Nakanishi et al. (2005), $2=$ Greve et al. (2009), $3=$ Krips et al. (2008), $4=$ Graciá-Carpio et al. (2008), $5=$ Papadopoulos et al. (2014), $6=$ Scoville et al. (2015), $\mathrm{x}=$ this work.

$J=4-3 \mathrm{HCN}$ and $\mathrm{HCO}^{+}$lines. Convolved source sizes range from $4^{\prime \prime}$ to $1^{\prime \prime} .5$ as frequency increases, with the deconvolved low $-J$ source sizes being quite uncertain, but with very compact high $-J$ deconvolved sizes: $1^{\prime \prime} .1 \times 0^{\prime \prime} .6$ for $\mathrm{HCN}(4-3)$ (Scoville et al. 2015). Even if the low-J emission is more widespread it will still be much smaller than the single dish beam sizes at the associated sky frequencies, so that the beam coupling corrections are $\lesssim 1.05$ and as such are much less significant than the single dish calibration uncertainties $(>10 \%)$. Also, this would be a systematic underestimate of the flux, so is accounted for to first order when using line ratios.

Source size: In all of the observations, except for the $\mathrm{HCN}(4-3)$ of Scoville et al. (2015), the source size is so uncertain that we cannot make any convincing estimates of the source size beyond the flat assumption that there is a common size to all $J$ levels. This could potentially introduce a bias into the models, with the lower- $J$ lines being integrated over a larger area than the high $-J$. However, this acts in the opposite sense to any potential beam coupling errors, which reduce the measured low $-J$ fluxes if the low $-J$ lines are significantly more extended. To some extent these opposing errors correct for any inaccuracies introduced by assuming a common source size and not attempting to account for changes in the beam coupling.

In addition to the observed line ratios we use the dynamical parameter $K_{v i r}$ to constrain our models (e.g., Greve et al. 2009; Papadopoulos et al. 2012, 2014), where

$$
K_{v i r}=\frac{\mathrm{d} v / \mathrm{d} r}{(\mathrm{~d} v / \mathrm{d} r)_{\text {vir }}}=\frac{1.54}{\sqrt{\alpha}} \frac{\mathrm{d} v}{\mathrm{~d} r}\left(\frac{n_{\mathrm{H}_{2}}}{1000 \mathrm{~cm}^{-3}}\right)^{-0.5} .
$$

The geometric coefficient $\alpha$ ranges from $1-2.5$ with an expectation value $\langle\sqrt{\alpha}\rangle=1.312$. The velocity gradient, $\mathrm{d} v / \mathrm{d} r$, describes the change in line-of-sight velocity through the cloud in $\mathrm{km} \mathrm{s}^{-1} \mathrm{pc}^{-1}$. In galactic GMCs in virial equilibrium $K_{v i r} \sim 1$. However, as was pointed out by Papadopoulos et al. (2014), in the extreme environments of LIRGs and ULIRGS a wider $K_{v i r}$ range is necessary to capture the true environment due to unusual GMCs and unbound gas. This is especially true given that we also model the shocked $\mathrm{CO}$ gas. We therefore follow Papadopoulos et al. (2014) and adopt limits of 0.5 and 20.0 on the allowed $K_{v i r}$ values for our models.

The modelling consisted of runs of increasing complex- 
TABLE 3

Three Phase Input Ranges. All Values $\log _{10}$

\begin{tabular}{lccc}
\hline Parameter & Hot, shocked & Diffuse & Cold, dense \\
\hline \hline$T_{\mathrm{k}}$ & $2.0-3.5$ & $1.0-3.0$ & $0.5-1.5$ \\
$n_{\mathrm{H}_{2}}$ & $1-6$ & $1-6$ & $4-8$ \\
$\mathrm{~d} v / \mathrm{d} r$ & $-1-3$ & \\
{$[\mathrm{CO}] /\left[{ }^{13} \mathrm{CO}\right]$} & \multicolumn{4}{c}{-3} & $1-3$ \\
\cline { 2 - 3 }$\left.\left[\mathrm{HC} / \mathrm{O}^{+}\right)\right]$ & - & - & \\
\hline$\left[\mathrm{H}^{13} \mathrm{C}\left(\mathrm{N} / \mathrm{O}^{+}\right)\right]$ & & $-8--2$ & $-12--4$ \\
$X_{\mathrm{CO}}$ & - & - & $-12--4$ \\
$X_{\mathrm{HCN}}$ & - & - & $-2-0$ \\
$X_{\mathrm{HCO}}+$ & $-2-0$ & - & \\
$f$ & & & \\
\hline
\end{tabular}

ity to check for consistency both with existing data and between models. Here, we present only the final model. The precursors are included in the Appendix.

The limits on the $\left[X_{12} \mathrm{C}\right] /\left[X_{13} \mathrm{C}\right]$ range were taken from the analysis of the $\mathrm{HCN}(1-0) / \mathrm{H}^{13} \mathrm{CN}(1-0)$ line ratio in Section 3.4 to be 10 and 1000 , while the limits on $T_{\mathrm{k}}, n_{\mathrm{H}_{2}}$ and $\mathrm{d} v / \mathrm{d} r$ are chosen to be consistent with Papadopoulos et al. (2014), although we extend the lower limit on $\mathrm{d} v / \mathrm{d} r$ to $0.1 \mathrm{~km} \mathrm{~s}^{-1} \mathrm{pc}^{-1}$ to better accommodate diffuse CO. The temperature of the background blackbody, $T_{\mathrm{bg}}$, is set to $3 \mathrm{~K}$. For models with free abundances we adopted $-12<\log _{10}\left(X_{\mathrm{mol}}\right)<-4$ for $\mathrm{HCN}$ and $\mathrm{HCO}^{+}$and $-8<$ $\log _{10}\left(X_{\mathrm{CO}}\right)<-2$ for CO.

\subsection{Three Phase Modelling}

A concern with simultaneously modelling $\mathrm{CO}, \mathrm{HCN}$ and $\mathrm{HCO}^{+}$is that about $30 \%$ of the $\mathrm{CO}(1-0)$ line flux is far more extended that any other molecular species, tracing an outflow out to almost $30^{\prime \prime}$, while the $\mathrm{HCN}$ and $\mathrm{HCO}^{+}$ emission only extends out to $\lesssim 2^{\prime \prime}$. (Feruglio et al. 2013; Nakanishi et al. 2005), potentially leading to significant errors and bias if we force it into the same gas phase as $\mathrm{HCN}$ and $\mathrm{HCO}^{+}$. We therefore adopt a three phase model consisting of a diffuse, extended $\mathrm{CO}$ phase, a hot and shocked CO phase and a cold, dense multi-species phase.

The parameter ranges are given in Table 3 . The temperature and density ranges are chosen so as to force the phases to be hot, diffuse or dense respectively, while still allowing a large range for each parameter to explore.

$\mathrm{HCN}$ and $\mathrm{HCO}^{+}$are only included in the cold, dense phase. This is motivated by the absence of any $\mathrm{HCN}$ emission from the shocked phase in the two phase models (Appendix C). There is a potential issue with excluding $\mathrm{HCO}^{+}$from the shocked and/or diffuse phase as it shows evidence in the precursor models of having a small but significant contribution from hotter and more diffuse gas. The choice to restrict $\mathrm{HCO}^{+}$to the dense phase is a pragmatic one: we simply do not have enough observed lines to constrain a more accurate model of $\mathrm{HCO}^{+}$. This should not significantly affect the results as the CO lines are the primary determinants of the conditions in the diffuse and shocked phases, and the warmer phase $\mathrm{HCO}^{+}$ emission is less than $10 \%$ of the flux in any of the lines. Furthermore, it is very unlikely to be present in significant quantities in the hot, shocked gas, nor in the fully extended diffuse phase; rather it is expected on the UV illuminated edges of molecular clouds, which we do not model at all.

$\mathrm{CO}$ is present in all three phases, and in each phase there is a free $[\mathrm{CO}] /\left[{ }^{13} \mathrm{CO}\right]$ ratio. In the cold, dense phase there is also an $[\mathrm{HCN}] /\left[\mathrm{H}^{13} \mathrm{CN}\right]$ ratio, which is shared with $\mathrm{HCO}^{+}$. Inspired by Zhang et al. (2014) and Kamenetzky et al. (2014) we relate the fluxes from the three phases through two free scaling factors $f_{1}$ and $f_{2}$, such that:

$$
S=S_{\text {shocked }} f_{1}+S_{\text {diffuse }}+S_{\text {dense }} f_{2} .
$$

It is important to appreciate that the $f$ 's are NOT the fractional flux densities emerging from the phases, as the fluxes from each phase may intrinsically be much greater (or smaller) than $S_{\text {diffuse }}$. They are merely free parameters to allow the MCMC to scale the significance of the two phases. Physically, they are indirectly related to the beam filling factors of the phases, whereas the formulation of Kamenetzky et al. (2014), who fitted line intensities instead of line ratios, used two beam filling factors directly as free parameters.

The contrast factors allow us to abstract away the issue that different molecular species present different beam filling factors. For the unresolved single dish observations this is essential, but even for high resolution interferometric observations this is a desirable property, allowing for that fact that the ISM can vary significantly over parsec scales (barely resolvable with ALMA in even the closest galaxies).

The results of the three phase modelling are shown in Figures 6, 7 and 8, while the numerical values are tabulated in Table 4. The model successfully splits into three clear phases, without any tension against the parameter ranges. $X_{\mathrm{CO}}$ varies greatly between the gas phases, being greatest in the diffuse phase lying near the canonical value of $2 \times 10^{-4}$, before dropping slightly to around $1 \times 10^{-5}$ in the shocked phase and then dropping rapidly to around $10^{-6}-10^{-7}$ in the cold, dense phase; strongly suggestive of freeze-out of $\mathrm{CO}$ onto dust grains in the cold, dense phase.

The isotopologue abundance ratios are also largely as we would expect: both the shocked and the dense gas phases have relatively low $[\mathrm{CO}] /\left[{ }^{13} \mathrm{CO}\right]$ ratios of $30-100$, likely representative of the true $\left[{ }^{12} \mathrm{C}\right] /\left[{ }^{13} \mathrm{C}\right]$ ratio in the galaxy. The diffuse phase present a slightly elevated ratio of $\sim 100-200$, consistent with this ubiquitous gas phase experiencing selective photodissociation due to the nuclear starbursts. Finally, the $[\mathrm{HCN}] /\left[\mathrm{H}^{13} \mathrm{CN}\right]$ ratio (recall that this is shared with $\mathrm{HCO}^{+}$) is the highest, at around 300 , consistent with isotope fractionation in cold, dense molecular cores.

The SLEDs in Figure 7 show exceptionally good fits to the observed lines. The cold, dense phase has very little contribution to the $\mathrm{CO}$ or ${ }^{13} \mathrm{CO}$ SLEDs, and in both of these the shocked phase dominates the emission at $J \gtrsim 4$, while the low $-J$ lines are set by the ubiquitous diffuse phase.

For each point in the MCMC trace we can calculate $K_{v i r}$ : the $K_{v i r}$ pdfs are shown in Figure 8. Both the diffuse and dense phases present doubly peaked pdfs, in particular with a peak about $K_{v i r}=1$, while the shocked phase pdf steadily increases towards the upper limit of $K_{v i r}=20 . \quad K_{v i r}$ is generally a poorly constrained parameter in our models, but these results are suggestive of approximately virialised gas dominating the diffuse and 
TABLE 4

Three Phase Results. All values $\log _{10}$. Values in PARENTHESIS ARE THE BEST FIT VALUES.

\begin{tabular}{lccc}
\hline Parameter & Hot, shocked & Diffuse & Cold, dense \\
\hline \hline$T_{\mathrm{k}}$ & $3.2_{3.1}^{3.3}(3.2)$ & $2.3_{1.8}^{2.9}(2.4)$ & $1.1_{1.0}^{1.3}(0.9)$ \\
$n_{\mathrm{H}_{2}}$ & $3.6_{3.4}^{3.9}(3.5)$ & $2.5_{2.2}^{2.7}(2.4)$ & $5.6_{5.0}^{6.5}(6.6)$ \\
$\mathrm{d} v / \mathrm{d} r$ & $0.8_{0.1}^{1.0}(0.2)$ & $0.1_{-0.6}^{0.4}(0.2)$ & $1.8_{1.1}^{1.9}(1.5)$ \\
$\frac{[\mathrm{CO}]}{\left[{ }^{13} \mathrm{CO}\right]}$ & $1.6_{1.5}^{1.9}(1.8)$ & $2.2_{1.9}^{2.4}(2.2)$ & $2.2_{1.6}^{2.8}(2.5)$ \\
$\frac{\left[\mathrm{HC}\left(\mathrm{N} / \mathrm{O}^{+}\right)\right]}{\left[\mathrm{H}^{13} \mathrm{C}\left(\mathrm{N} / \mathrm{O}^{+}\right)\right]}$ & - & - & $2.5_{2.3}^{2.7}(2.6)$ \\
$X_{\mathrm{CO}}$ & $-5.0_{-5.4}^{-4.5}(-5.1)$ & $-3.8_{-5.4}^{-2.7}(-3.7)$ & $-6.6_{-7.5}^{-5.6}(-7.4)$ \\
$X_{\mathrm{HCN}}$ & - & - & $-8.0_{-7.9}^{-7.2}(-9.6)$ \\
$X_{\mathrm{HCO}}$ & - & - & $-8.8_{-9.0}^{-8.0}(-10.2)$ \\
$f$ & $-0.5_{-0.7}^{-0.3}(-0.6)$ & - & $-0.7_{-1.0}^{-0.5}(-0.2)$ \\
Mass $\left[M_{\odot}\right]^{\mathrm{a}}$ & $9.4_{8.9}^{9.8}(9.1)$ & $9.3_{8.6}^{9.9}(8.7)$ & $10.3_{9.6}^{10.8}(10.0)$ \\
\hline
\end{tabular}

${ }^{\mathrm{a}} \mathrm{CO}$ estimated mass from calculated $\alpha_{\mathrm{CO}}$ and the observed $\mathrm{CO}(1-0)$ line.

dense gas phases, while the shocked phase is highly turbulent.

We also show the $[\mathrm{HCN}] /\left[\mathrm{HCO}^{+}\right]$abundance ratio pdf in Figure 9. This ratio is very tightly constrained as $6.2_{4.3}^{7.9}$.

We can also calculate $\alpha_{\operatorname{mol}(1-0)}$, where $\alpha_{\operatorname{mol}(1-0)}=$ $M_{\text {dense }} / L_{\operatorname{mol}(1-0)}^{\prime}$ is the ratio of the gas mass of the phase to the $J=1-0$ line luminosity of the species from that phase $^{12}$. We use equation A4 for optically thick gas from Papadopoulos et al. $(2012)^{13}$ to derive $\alpha_{\operatorname{mol}(1-0)}$ for CO and HCN and present the pdfs, along with the corresponding masses, in Figure 10. The spread in each of the $\alpha_{\mathrm{CO}}$ pdfs is between 1.5 and 2 dex, with the centres shifting to increasing $\alpha_{\mathrm{CO}}$ as we move from the diffuse phase, through the shocked phase and onto the dense phase. There is less spread in the masses, and in particular the HCN derived and $\mathrm{CO}$ derived dense gas masses are very consistent with almost identical pdfs but for a systematic shift of $\sim-0.2$ dex on the $\mathrm{CO}$ mass estimates. These are discussed in Section 5.3.

Interestingly, the thermal pressure of the shocked and dense gas phases is $P / k \sim 10^{7} \mathrm{~K} \mathrm{~km} \mathrm{~s}^{-1}$ - the same value that Dopita et al. (2005) found necessary to fit the SED in NGC 6240 and the value found for the hot, diffuse phase in Kamenetzky et al. (2014). This makes sense in light of the three phase model: the diffuse $\mathrm{CO}$ and shocked CO dominate the CO SLED, so when Kamenetzky et al. (2014) fitted to the CO SLED only they recovered these phases. However, the diffuse phase is only partially co-located with the shocked phase so is not in pressure balance, whereas the dense phase, identified here by fitting $\mathrm{HCN}, \mathrm{HCO}^{+}$and $\mathrm{CO}$ simultaneously, is co-located with the shocked phase, and approximately in pressure balance with it. On the other hand, we would expect a significant fraction of the pressure balance to be due to macroscopic turbulence, not just thermal pressure.

$$
\begin{aligned}
& { }^{12} \text { Units of } M_{\odot}\left(\mathrm{K} \mathrm{km} \mathrm{s}^{-1} \mathrm{pc}^{2}\right)^{-1} . \\
& { }^{13} \alpha_{\operatorname{mol}(1-0)}=\frac{3.25}{\sqrt{\alpha}} \frac{\sqrt{\left\langle n_{\mathrm{H}_{2}}\right\rangle}}{\left\langle T_{\mathrm{b}, 1-0}\right\rangle} K_{v i r}^{-1}
\end{aligned}
$$

\subsection{Comparison to the Literature}

The dense, HCN bearing molecular gas in NGC 6240 has been modelled previously, by Krips et al. (2008), Greve et al. (2009) and Papadopoulos et al. (2014) (Kamenetzky et al. 2014 only modelled the CO SLED). While Krips et al. (2008) modelled a single gas phase for both $\mathrm{HCN}_{\text {and }} \mathrm{HCO}^{+}$, Greve et al. (2009) and Papadopoulos et al. (2014) fitted $\mathrm{HCN}$ and $\mathrm{HCO}^{+}$independently, each with their own gas phase parameters.

While Krips et al. (2008) and Greve et al. (2009) both found similar gas kinetic temperature ranges $(20-120 \mathrm{~K}$ and $60-120 \mathrm{~K}$ respctively), Greve et al. (2009) found much larger $n_{\mathrm{H}_{2}}$ in the HCN phase of $\sim 10^{5} \mathrm{~cm}^{-3}$, cf. $10^{3.5}-10^{4.5} \mathrm{~cm}^{-3}$ found by Krips et al. (2008). On the other hand, the $\mathrm{HCO}^{+}$phase had an $n_{\mathrm{H}_{2}}$ of $\sim 10^{4} \mathrm{~cm}^{-3}$, consistent with the results of Krips et al. (2008).

Papadopoulos et al. (2014) present their results as 2D pdfs, finding degenerate "bananas" ranging from $n_{\mathrm{H}_{2}} \simeq$ $10^{6} \mathrm{~cm}^{-3} / T_{\mathrm{k}} \simeq 8 \mathrm{~K}$ to $n_{\mathrm{H}_{2}} \simeq 10^{4} \mathrm{~cm}^{-3} / T_{\mathrm{k}} \simeq 1000 \mathrm{~K}$, although the $\mathrm{HCN}$ pdf appears to peak at the high-density, low- $T_{\mathrm{k}}$ end of the "banana".

Our results are broadly consistent with these previous works. Both our precursor models and the full three phase model argue for relatively cool $(8-30 \mathrm{~K})$, dense $\left(10^{4.5}-10^{6.5} \mathrm{~cm}^{-3}\right)$ gas dominating the $\mathrm{HCN}$ and $\mathrm{HCO}^{+}$ emission. These models echo the trend seen by Greve et al. (2009) and Papadopoulos et al. (2014) of $\mathrm{HCO}^{+}$ preferring a $\sim 0.5$ dex lower $n_{\mathrm{H}_{2}}$ than $\mathrm{HCN}$ while sharing a similar kinetic temperature. Our $[\mathrm{HCN}] /\left[\mathrm{HCO}^{+}\right]$ abundance ratio of $5.3_{4.7}^{7.0}$ is consistent with Krips et al. (2008) who found "around 10".

The multiple phases of the molecular gas in NGC 6240 have been characterised previously, including by Armus et al. (2006) with Spitzer observations of $\mathrm{H}_{2}$ vibrational lines, Papadopoulos et al. (2014) with the aforementioned spectral decomposition of the CO SLED and Kamenetzky et al. (2014) with a two phase MCMC model of the CO SLED. Our models are the first to simultaneously fit multiple species in multiple gas phases. As was pointed out by Kamenetzky et al. (2014), and as we found here comparing to the results of Papadopoulos et al. (2014), it is important to simultaneously fit multiple components to SLEDs to prevent the biasing of results.

From modelling of $\mathrm{H}_{2}$ vibrational lines Armus et al. (2006) found two highly excited phases of the molecular gas, with $6.7 \times 10^{6} M_{\odot}$ at $T_{\mathrm{k}}=957 \mathrm{~K}$ and $1.6 \times 10^{9} M_{\odot}$ at $T_{\mathrm{k}}=164 \mathrm{~K}$ respectively. Papadopoulos et al. (2014) first fitted the $\mathrm{HCN}$ and $\mathrm{HCO}^{+}$SLEDs, before using these results to fit high $-J$ components of the CO SLED, then fitting the residual CO SLED. This produces three gas phases with densities $\log _{10}\left(n_{\mathrm{H}_{2}}\right)=5.0,4.3$ and 3.0 and kinetic temperatures $\log _{10}\left(T_{\mathrm{k}}\right)=1.5,2.6$ and 2.0. The model of Kamenetzky et al. (2014) used a nested sampling algorithm to fit two gas phases to the $\mathrm{CO}$ SLED, finding a hot and a cold phase with best fit densities $\log _{10}\left(n_{\mathrm{H}_{2}}\right)=4.1$ and 5.8 and kinetic temperatures $\log _{10}\left(T_{\mathrm{k}}\right)=3.1$ and 1.2 . These are reassuringly similar to the hot, shocked and cold, dense phase results of our multi-species, multi-phase analysis. It is curious however that the cold phase of their two phase model was closer to the fainter cold, dense phase than the brighter diffuse 


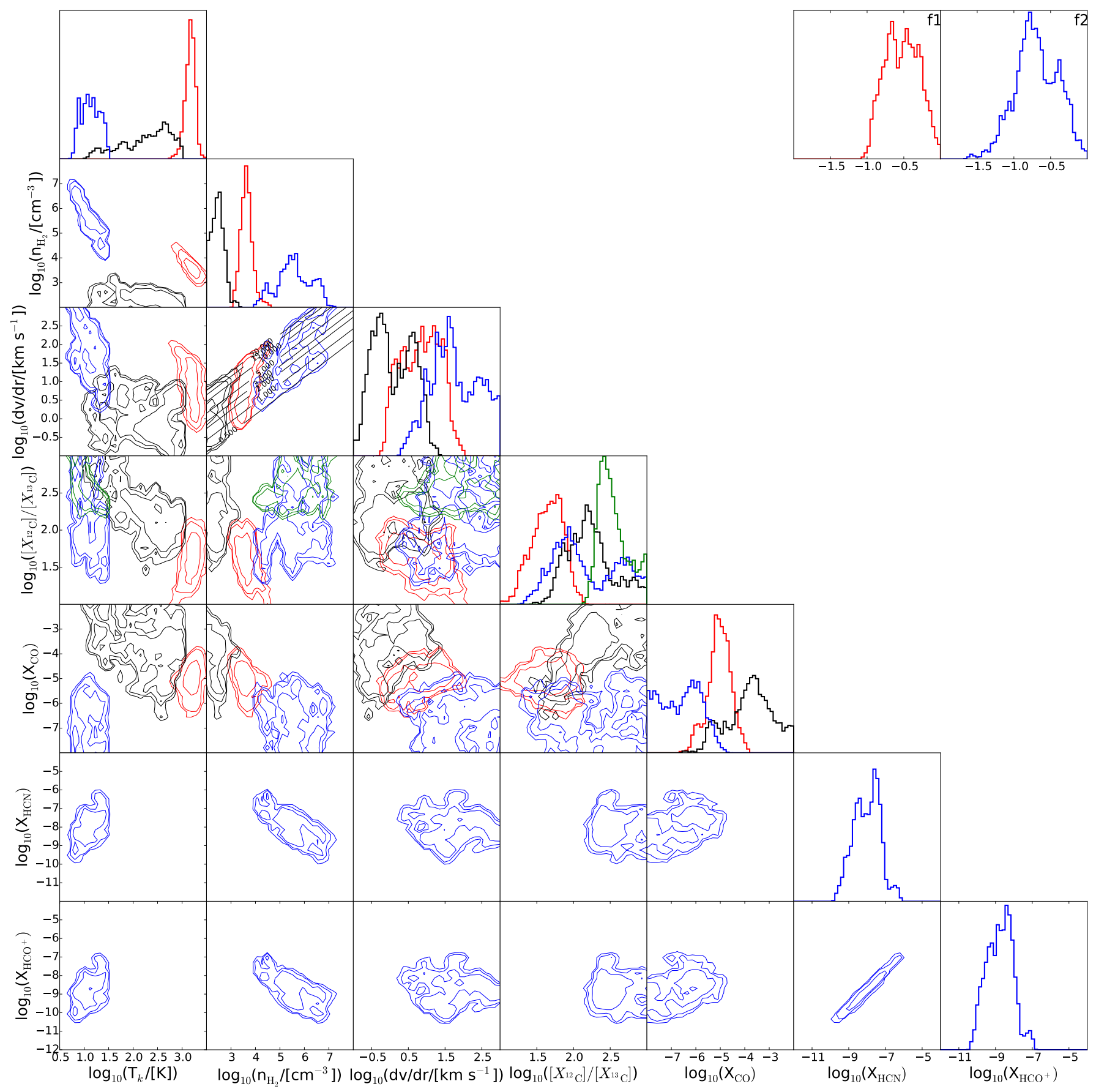

FIG. 6. - The step plot for the three phase $\mathrm{HCN}+\mathrm{HCO}^{+}+\mathrm{CO}$ model results. Contours are at the $68 \%, 95 \%$ and $99 \%$ credible intervals. Red, black and blue contours are the shocked, diffuse and dense gas phases respectively. In the $\left[X_{12} \mathrm{C}\right] /\left[X_{13} \mathrm{C}\right]$ plots the green contours correspond to the $[\mathrm{HCN}] /\left[\mathrm{H}^{13} \mathrm{CN}\right]$ abundance ratio (shared with $\mathrm{HCO}^{+}$), and the blue the $[\mathrm{CO}] /\left[{ }^{13} \mathrm{CO}\right]$ ratio, both in the cold, dense phase.

CO phase. There is no clear explanation for this, but it could very possibly be an artefact of the change from two to three phases.

\section{DISCUSSION}

\subsection{Validity of Multi-species Models}

An important question of our analysis is whether or not it is appropriate to model multiple species as sharing the same gas phase. In reality there is a continuum of conditions within any telescope beam and any phase based model is only an approximation of the conditions dominating the emission, which will vary from molecule to molecule. Nevertheless, combining molecular species which are expected to trace similar regions appears to be a powerful tool for estimating molecular abundance ratios and gas conditions.

The combination of two or more species in a single model is a compromise, with the extent of the assumptions dependent upon how similar the regions traced by the different species are likely to be. In our three phase model, the dense phase is shared by $\mathrm{HCN}, \mathrm{HCO}^{+}$ and $\mathrm{CO}$, as well as their ${ }^{13} \mathrm{C}$ isotopologues. That $\mathrm{CO}$ 

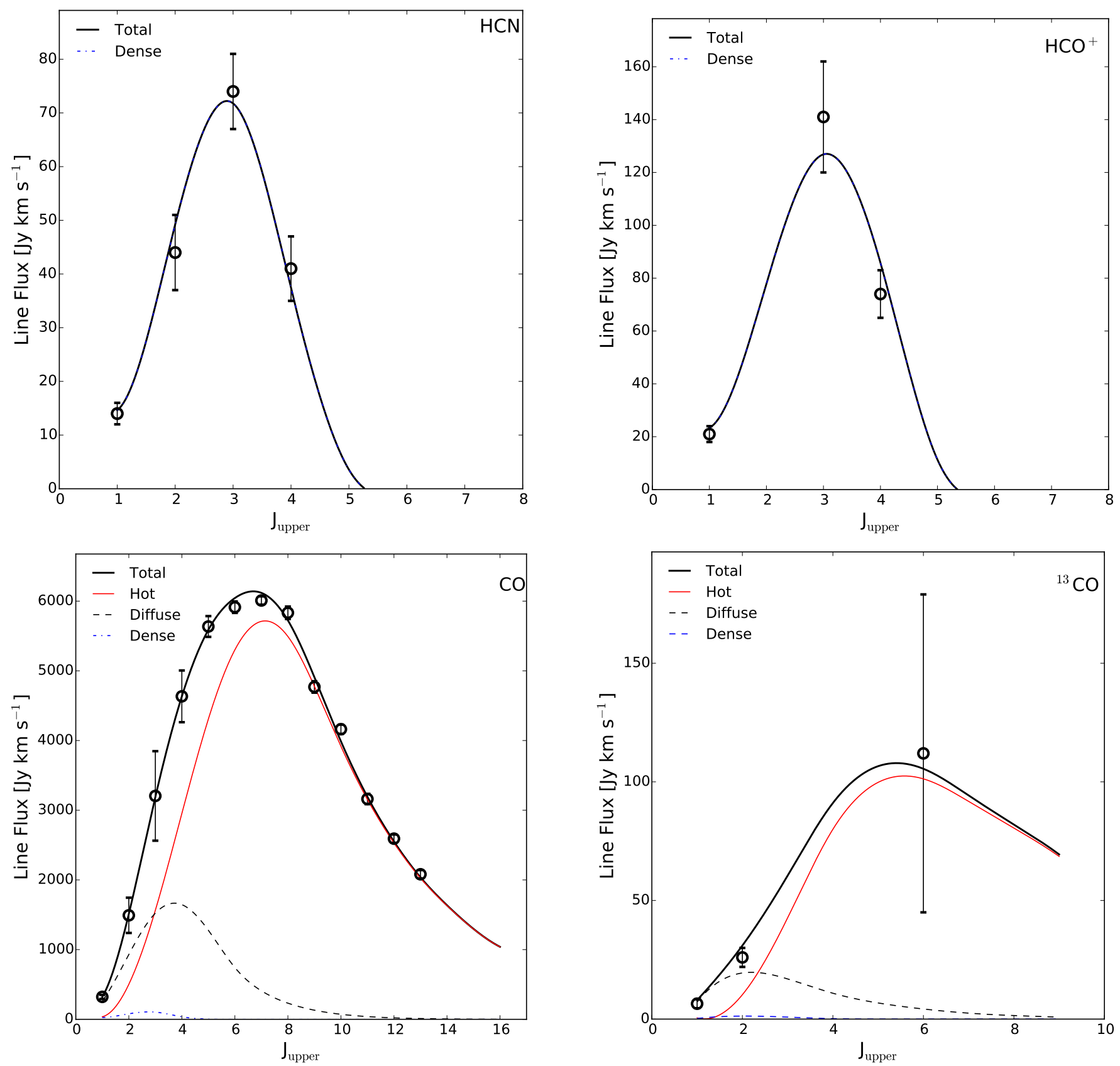

FIG. 7.- SLED fits for the three phase $\mathrm{HCN}+\mathrm{HCO}^{+}+\mathrm{CO}$ model, showing the relative contribution of each gas phase. The same normalisation has been used for the $\mathrm{CO}$ and ${ }^{13} \mathrm{CO}$ SLEDs.

is present alongside other molecules goes without question, even if its abundance is reduced due to freeze-out onto ice grains, and the other two gas phases prevent $\mathrm{CO}$ from being heavily biased by the dense conditions; the colocation of $\mathrm{HCN}$ and $\mathrm{HCO}^{+}$is less concrete. While $\mathrm{HCN}$ and $\mathrm{HCO}^{+}$are almost certainly colocated, $\mathrm{HCO}^{+}$ is quite possibly more extended, tracing warmer and less UV shielded regions (Fuente et al. 2005). On the other hand, it is not expected to be found in the very diffuse and extended CO gas phase we use here. Unfortunately, while an additional phase would be desirable we would not be able to place meaningful constraints upon the additional phase due to the paucity of lines. Two phase $\mathrm{HCN}$ and $\mathrm{HCO}^{+}$models (Appendix C.3) suggest that the contribution of $\mathrm{HCO}^{+}$in a slightly hotter and less dense phase (physically this would most likely correspond to the transition region between the hot, diffuse phase and the cold, dense cores) is less than $10 \%$ of any $\mathrm{HCO}^{+}$ line. We believe that the colocation of $\mathrm{HCN}$ and $\mathrm{HCO}^{+}$ in the cold, dense phase is therefore an undesirable but necessary, and acceptable, compromise.

\subsection{The $\left[{ }^{12} C\right] /\left[{ }^{13} C\right]$ Abundance Ratio in NGC6240}

Papadopoulos et al. (2014) constrained the high $-J$ CO lines using the LVG solutions for $\mathrm{HCN}$ and $\mathrm{HCO}^{+}$before fitting a third phase to the remaining $\mathrm{CO}$ lines. When they then applied these three phases to their ${ }^{13} \mathrm{CO}$ data, a very high $[\mathrm{CO}] /\left[{ }^{13} \mathrm{CO}\right]$ abundance ratio of $300-500$ was required for consistency and they argued for that this was evidence for a similarly elevated $\left[{ }^{12} \mathrm{C}\right] /\left[{ }^{13} \mathrm{C}\right]$ ra- 


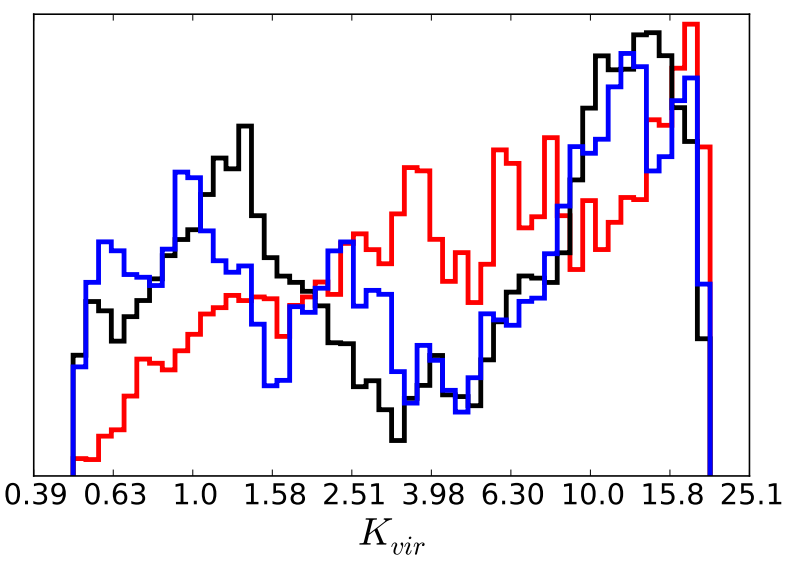

FIG. 8.- The $K_{v i r}$ pdfs for the three phase model. Colours are as in Figure 6, with red, black and blue correspond to the shocked, diffuse and dense phases respectively. Both the diffuse and dense phases present doubly peaked pdfs, in particular with a peak about $K_{v i r}=1$, while the shocked phase pdf steadily increases towards the upper limit of $K_{v i r}=20$.

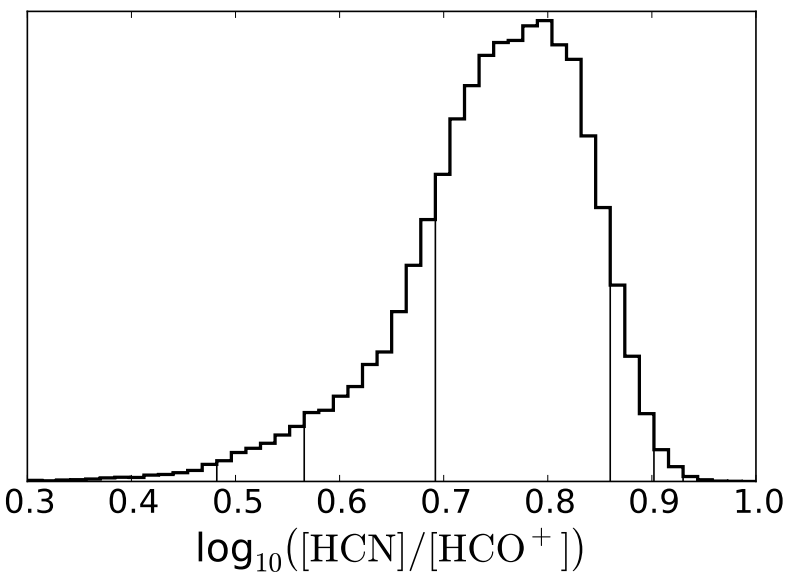

FIG. 9. - The $[\mathrm{HCN}] /\left[\mathrm{HCO}^{+}\right]$abundance ratio pdf from the three phase model. The value is tightly constrained as $6.2_{4.3}^{7.9}$.

tio. Here, we have used our new observations of $\mathrm{H}^{13} \mathrm{CN}$ and $\mathrm{H}^{13} \mathrm{CO}^{+}$in combination with the $\mathrm{CO}$ and ${ }^{13} \mathrm{CO}$ lines from the literature to place new constraints on the $\left[{ }^{12} \mathrm{C}\right] /\left[{ }^{13} \mathrm{C}\right]$ abundance ratio. We have found that instead of arising in the $\mathrm{HCN}$ and $\mathrm{HCO}^{+}$bearing dense gas, the high $-J$ CO lines are due to the hot, shocked gas phase, which in turn changes the implied $[\mathrm{CO}] /\left[{ }^{13} \mathrm{CO}\right]$ ratios.

A naïve approach, simply taking the $68 \%$ credible intervals on $[\mathrm{CO}] /\left[{ }^{13} \mathrm{CO}\right]$ and $[\mathrm{HCN}] /\left[\mathrm{H}^{13} \mathrm{CN}\right]$ as the brackets on the $\left[{ }^{12} \mathrm{C}\right] /\left[{ }^{13} \mathrm{C}\right]$ ratio, places the ratio somewhere between 100 and 1000: however, this does not account for the much greater abundance of $\mathrm{CO}$ wrt $\mathrm{HCN}$ nor the dominance by mass of the dense phase.

We adopt a mass and abundance weighted average and find $\left[{ }^{12} \mathrm{C}\right] /\left[{ }^{13} \mathrm{C}\right]=98_{65}^{230}$. The pdf of the ratio is shown in Figure 11. The ratio is strongly peaked at "normal" ULIRG values of about 100 , although there is an extended tail out to 1000 . The model is compatible within $-1 \sigma$ with a Galactic value, while the $+3 \sigma$ boundary is at 550. We therefore argue against a super-ULIRG elevated $\left[{ }^{12} \mathrm{C}\right] /\left[{ }^{13} \mathrm{C}\right]$ abundance ratio, but cannot conclusively exclude the range found by Papadopoulos et al. (2014). The ideal solution, a full chemical-hydrodynamical model with radiation transfer, is beyond the scope of this paper. Nevertheless, it appears that the $\left[{ }^{12} \mathrm{C}\right] /\left[{ }^{13} \mathrm{C}\right]$ ratio in NGC 6240 is most similar to other ULIRGs.

Are the derived isotopologue abundance ratios consistent with the chemical models presented in Section 1.1? Given the wide range of results from the chemical models, and their strong dependences on temperature, density, metallicity, the $\mathrm{C} / \mathrm{O}$ ratio and the $\mathrm{H}_{2}$ ortho-para ratio it is hard to say with certainty. We find $[\mathrm{HCN}] /\left[\mathrm{H}^{13} \mathrm{CN}\right]$ $\sim 2 \times[\mathrm{CO}] /\left[{ }^{13} \mathrm{CO}\right]$ in the dense and diffuse gas phases, consistent with Roueff et al. (2015). The rather high $\left[\mathrm{HCO}^{+}\right] /\left[\mathrm{H}^{13} \mathrm{CO}^{+}\right]$(which in the three phase model is fixed to be the same as $[\mathrm{HCN}] /\left[\mathrm{H}^{13} \mathrm{CN}\right]$ ) is surprising, but not so extreme as to cause concern; Langer et al. (1984) found $\left[\mathrm{HCO}^{+}\right] /\left[\mathrm{H}^{13} \mathrm{CO}^{+}\right] \sim 1.5 \times[\mathrm{CO}] /\left[{ }^{13} \mathrm{CO}\right]$ in $10 \mathrm{~K}, 10^{4} \mathrm{~cm}^{-3}$, low metallicity gas. Furthermore, since this ratio is fixed to $[\mathrm{HCN}] /\left[\mathrm{H}^{13} \mathrm{CN}\right]$ this could be artificially elevating it (see Figure 17 in the Appendix).

The $[\mathrm{CO}] /\left[{ }^{13} \mathrm{CO}\right]$ ratio appears to increase from $40_{30}^{80} \mathrm{in}$ the hot phase to $160_{40}^{630}$ in the cold, dense phase. There is slight tension between these results and our interpretation of the model results as being evidence for ICE: in the case of ICE fractionation in the cold, dense phase should lower the $[\mathrm{CO}] /\left[{ }^{13} \mathrm{CO}\right]$ ratio; it is therefore contradictory if the hot, shocked phase, where there is no isotope fractionation, presents a lower $[\mathrm{CO}] /\left[{ }^{13} \mathrm{CO}\right]$ ratio than the dense phase. However, there is still a region of parameter space within the $68 \%$ credible interval about the mean values where the $[\mathrm{CO}] /\left[{ }^{13} \mathrm{CO}\right]$ ratio is lower in the dense phase, so while there is tension it is not that great, and the model is still consistent with isotope fractionation. Furthermore, the hot phase $[\mathrm{CO}] /\left[{ }^{13} \mathrm{CO}\right]$ is almost entirely determined by the highly uncertain ${ }^{13} \mathrm{CO} J=6-5$ line, while the dense phase $\mathrm{CO}$ and ${ }^{13} \mathrm{CO}$ emission is almost zero (Figure 7 ), so that the $[\mathrm{CO}] /\left[{ }^{13} \mathrm{CO}\right]$ ratio is highly susceptible to small errors. The $[\mathrm{CO}] /\left[{ }^{13} \mathrm{CO}\right]$ vs $T_{\mathrm{k}}$ panel of Figure 6 shows both that there is a large spread in all of the $[\mathrm{CO}] /\left[{ }^{13} \mathrm{CO}\right]$ values, and that there is a very large overlapping region within the $68 \%$ credible intervals. Interestingly, within this spread in $[\mathrm{CO}] /\left[{ }^{13} \mathrm{CO}\right]$ all three phases are consistent with a value of $\sim 80$, closer to the $\sim 70$ of the Milky Way than to a globally elevated elemental $\left[{ }^{12} \mathrm{C}\right] /\left[{ }^{13} \mathrm{C}\right]$ ratio.

It is possible that due to insufficient UV-plane sampling we have lost some of the continuum and line fluxes, as was mentioned in Section 3.1. The effect of this would be to bias our $[\mathrm{HCN}] /\left[\mathrm{H}^{13} \mathrm{CN}\right]$ and $\left[\mathrm{HCO}^{+}\right] /\left[\mathrm{H}^{13} \mathrm{CO}^{+}\right]$to higher values; our finding that the elemental $\left[{ }^{12} \mathrm{C}\right] /\left[{ }^{13} \mathrm{C}\right]$ ratio is not globally elevated in NGC 6240 is therefore robust against this potential source of error, as the error would push the results towards an elevated value.

\subsection{Line Luminosity to Gas Mass Conversion Factors}

The ratio between line luminosity and gas mass, $\alpha$, is of great interest as it provides a means of estimating galaxy gas fractions from observations of a single molecular line, usually CO. However, the determination of a general $\alpha_{\mathrm{CO}}$ is particularly complicated, with dependences on luminosity (Solomon et al. 1987) and metallicity, leading to a range of values from 0.3 to almost 300 , and a typical 

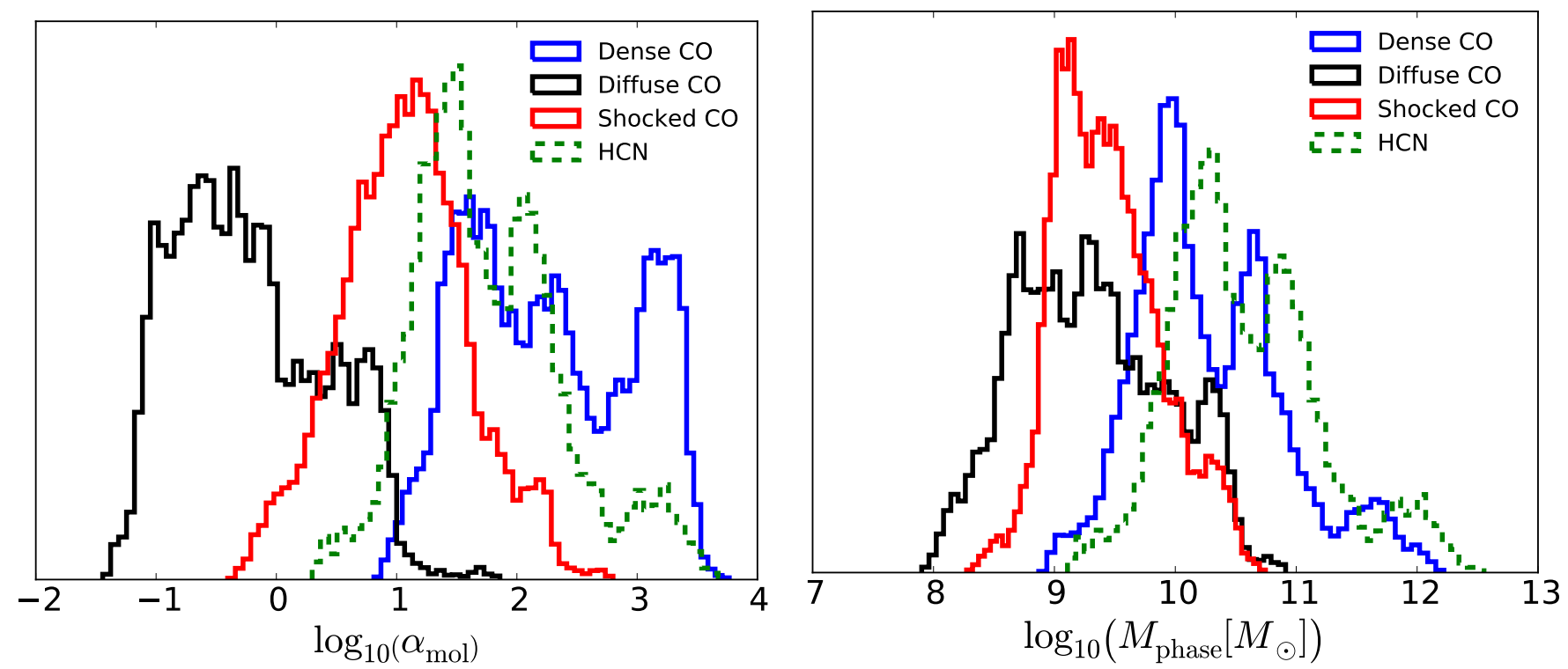

FIG. 10. - The $\alpha_{\mathrm{CO}}$ factor for each of the gas phases of the three phase model and the $\alpha_{\mathrm{HCN}}$ (encompassing the dense phase) (left), and the corresponding mass of the gas phase (right). The diffuse and shocked phases are consistent with the "standard" $\alpha_{\mathrm{CO}}$ values of between 0.5 and 4 , while the dense gas phase is considerably higher. While contributing a very small fraction of the $\mathrm{CO}(1-0)$ line flux the dense gas phase dominates the molecular gas mass in the galaxy. The CO estimate and the HCN estimate of the mass of the dense phase are highly consistent.

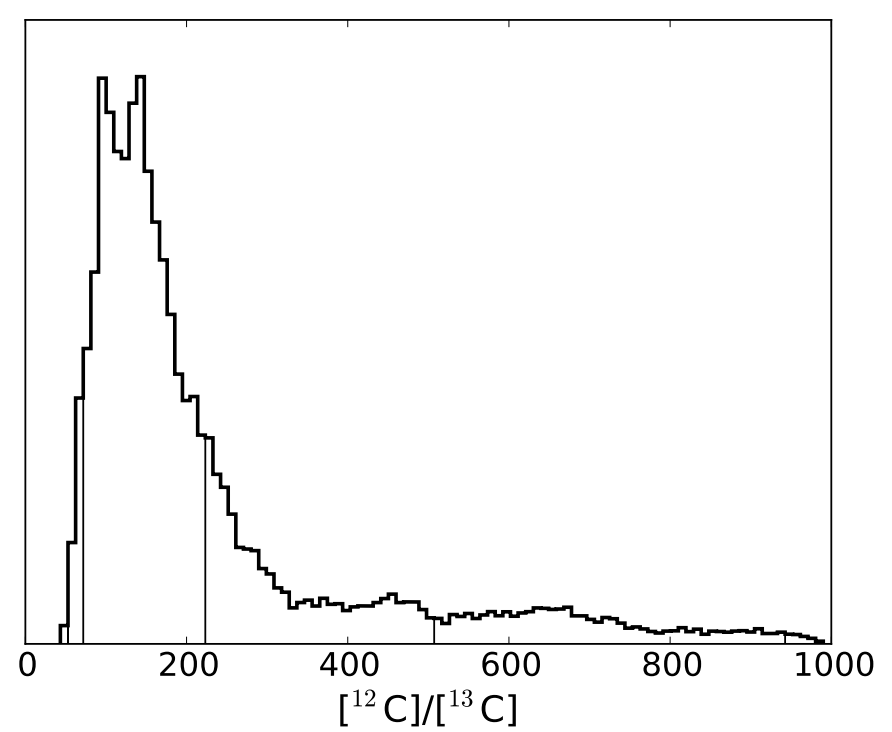

FIG. 11. - The pdf for the $\left[{ }^{12} \mathrm{C}\right] /\left[{ }^{13} \mathrm{C}\right]$ ratio in NGC 6240 , derived from the three phase model in Section C.5 using a mass and abundance weighted average. The ratio shows signs of being slightly elevated, but the upper $68 \%$ credible interval is less than half the value found by Papadopoulos et al. (2014) and the pdf is most consistent with the "normal" ULIRG abundance ratio.

Milky Way value of 4.8 dropping to about 1 in $z=2$ SMGs and 0.8 in (U)LIRGs ${ }^{14}$ (Downes \& Solomon 1998; Bolatto et al. 2013). Nevertheless, it has recently been argued that $\alpha_{\mathrm{CO}}$ may be underestimated in (U)LIRGs, and a Galactic value may be more accurate (Papadopoulos et al. 2012), due to gas over-densities arising from over-pressurisation due to turbulence.

There is interest in $\alpha_{\mathrm{HCN}}$ as a possible estimator of the dense molecular gas mass, which may be more ro-

\footnotetext{
14 Downes \& Solomon (1998) found a range for $\alpha_{\mathrm{CO}}$ from 0.3 to 1.3 in ULIRGs.
}

bust over a wide range of luminosities and may more accurately trace the specifically star forming gas (Gao \& Solomon 2004; Greve et al. 2009; García-Burillo et al. 2012). This in turn has been complicated by high resolution studies of ULIRGs which suggest that HCN emission may be dominated by shocked, X-ray heated or infrared pumped emission (Aalto et al. 2007; Sakamoto et al. 2010; Aalto et al. 2012; García-Burillo et al. 2014; Viti et al. 2014; Aalto et al. 2015; Martín et al. 2015; Tunnard et al. 2015).

We calculate $\alpha_{\text {mol }}$ and corresponding mass pdfs for each gas phase. The values for our three phase model are shown in Figure 10 where we plot $\alpha_{\mathrm{CO}}$ for each of the three gas phases, and $\alpha_{\mathrm{HCN}}$ for the dense phase, as well as the corresponding masses. The $\alpha_{\mathrm{CO}}$ pdfs are broad, with spans of about $1.5-2$ dex. The diffuse and shocked phases both cover the range $\alpha_{\mathrm{CO}}=1-10$, while the diffuse phase extends down to 0.1. The dense phase $\alpha_{\mathrm{CO}}$ is much higher, due to a lower $X_{\mathrm{CO}}$, higher $n_{\mathrm{H}_{2}}$ and higher $\mathrm{d} v / \mathrm{d} r$ in this phase, all of which contribute to an increased $\alpha_{\mathrm{CO}}$. Despite contributing $<5 \%$ of the $\mathrm{CO}(1-0)$ line flux, the dense phase accounts for almost all of the molecular gas mass. The dense phase gas masses derived using $\mathrm{HCN}$ and using $\mathrm{CO}$ are consistent but for an unexplained systematic offset of -0.2 dex on the $\mathrm{CO}$ mass estimate. $\alpha_{\mathrm{HCN}}=32_{13}^{89}$ : higher than the canonical value of 10 but similar to the $13-31.1$ found by Papadopoulos et al. (2014), although some regions of their Figure 14 do extend to $\alpha_{\mathrm{HCN}}=60$. Gao \& Solomon (2004) predicted $\alpha_{\mathrm{HCN}} \sim 25$ for $X_{\mathrm{HCN}}=2 \times 10^{-8}$ and $\mathrm{d} v / \mathrm{d} r=$ $5 \mathrm{~km} \mathrm{~s}^{-1} \mathrm{pc}^{-1}$ : our lower $X_{\mathrm{HCN}}$ and higher $\mathrm{d} v / \mathrm{d} r$ are responsible for our slightly higher $\alpha_{\mathrm{HCN}}$. While the $+1 \sigma$ level is very high, the best fit and $-1 \sigma$ level are consistent with Greve et al. (2009), who found $\alpha_{\mathrm{HCN}}=17-37$ in NGC 6240.

We derive a global $\alpha_{\mathrm{CO}}$ by calculating the sum of the masses of each gas phase, and dividing by the total 


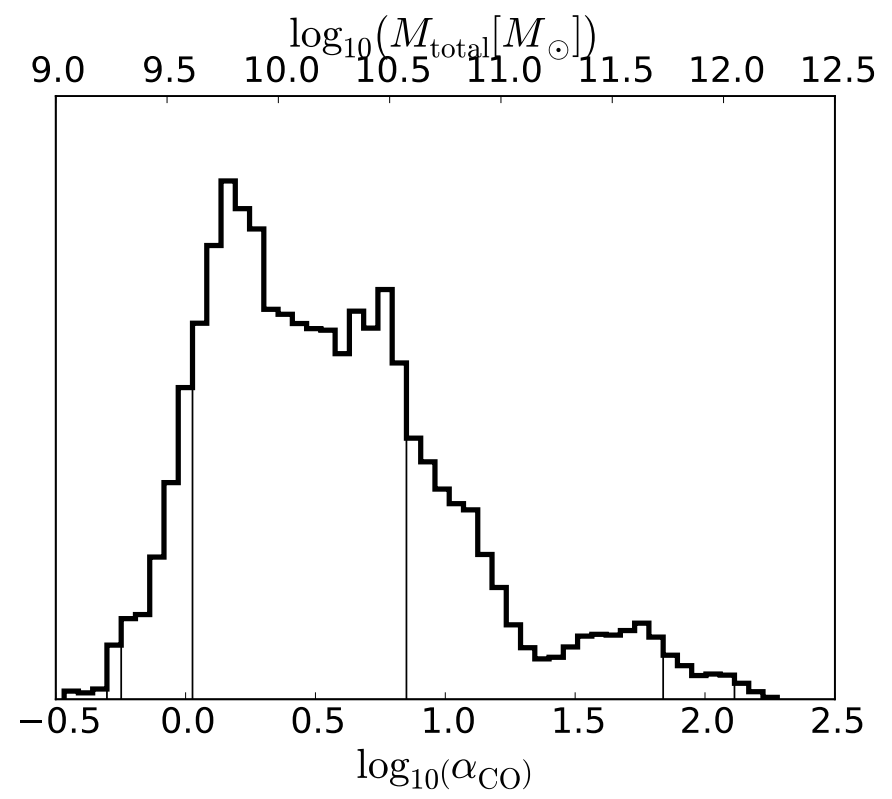

FIG. 12.- The global $\alpha_{\mathrm{CO}}$ for NGC 6240 derived from the three phase model with the corresponding total molecular gas mass.

$\mathrm{CO}(1-0)$ line luminosity. The resultant $\alpha_{\mathrm{CO}} \mathrm{pdf}$ and the corresponding mass pdf for NGC 6240 is shown in Figure 12. This global $\alpha_{\mathrm{CO}}$ is much more tightly constrained than the those of the individual phases, and we find $\alpha_{\mathrm{CO}}=1.5_{1.1}^{7.1}\left(\log _{10}\left(M /\left[M_{\odot}\right]\right)=10.1_{10.0}^{10.8}\right)$ : consistent with a Galactic $\alpha_{\mathrm{CO}}$, as predicted by Papadopoulos et al. (2012), but perhaps more consistent with the canonical $\alpha_{\mathrm{CO}}$ of starbursts (0.8, Downes \& Solomon 1998). Notably, the very low (CO) luminosity dense phase contributes the majority of the gas mass.

The high $\alpha_{\mathrm{HCN}}$ we derive is contrary to the findings of García-Burillo et al. (2012), who argued for a lower $\alpha_{\mathrm{HCN}}$ in (U)LIRGs, matching the lower $\alpha_{\mathrm{CO}}$. It is very possible that this contradiction is simply due to the extremely unusual properties of NGC 6240; NGC 6240 is perhaps not even representative of other (U)LIRGs, existing in a class of its own.

\subsection{Dense Gas and Star Formation in NGC 6240}

The results of our LVG models present an intriguing picture of the dense molecular gas in NGC 6240. We appear to be finding large quantities of very cold $(\sim 8 \mathrm{~K})$ gas within the internuclear molecular disk, which is also host to extensively shocked and very hot $(\sim 2000 \mathrm{~K})$ molecular gas. The question is how can such a large quantity of cold, dense gas coexist with the hot phase. Furthermore, star formation in NGC 6240 is not found in the internuclear molecular disk; it is instead concentrated in the two nuclei. If we truly have almost $10^{10} M_{\odot}$ of cold, dense gas then we must be able to explain why this gas is not actively forming stars.

An insight is offered if we apply an upper limit to the $\mathrm{H}_{2}$ column density of the dense phase. There is an inherent degeneracy within the LVG model between a single, large cloud and multiple, small clouds distributed in velocity space across the large scale velocity field of the galaxy. However, for the dense gas phase a single cloud implies column densities in excess of $10^{26} \mathrm{~cm}^{-2}$, which are extremely unlikely. If instead we assume that the column densities of the clouds are $\simeq 10^{23} \mathrm{~cm}^{-2}$ then we are instead seeing multiple small $(0.1-0.01 \mathrm{pc})$ dense clouds.

We therefore propose the following situation. Within the turbulent internuclear disk instabilities lead to rapid, localised cooling of pockets of gas, leading to multiple small, cold, dense clouds embedded in pressure equilibrium with the hot, diffuse phase but radiatively decoupled due to turbulent Doppler shifting of the lines. This rapid cooling leads to an evolving chemistry, potentially with molecules less stable than $\mathrm{CO}$ destroyed in the high temperatures of the shocks reforming as the gas cools. These clouds are still turbulent however, and may indeed be transient, explaining the lack of star formation.

In the three phase model the CO abundance is significantly lower in the cold, dense phase than in the hot phase or diffuse phase: we have almost a continuum of $X_{\mathrm{CO}}$ from the the diffuse phase value of $\sim 10^{-4}$, falling to $\sim 10^{-5}$ in the shocked phase and then to $\sim 10^{-6}$ in the dense phase. This is consistent with the many cold clouds interpretation above, with freeze out of $\mathrm{CO}$ onto dust grain mantles depleting the gas phase $\mathrm{CO}$ in cold, dense clouds - as observed in Milky Way cold cores (Kramer et al. 1999; Tafalla et al. 2002; Liu et al. 2013; Ripple et al. 2013). This might also explain the very strangely hot and diffuse CS model solutions from the precursor models (Table 7): CS is depleted along with CO (Tafalla et al. 2002), so the only CS we observe is in the hot gas phase. Unfortunately this is highly speculative, and cannot be confirmed without much higher spatial resolution observations able to resolve the high density molecular clouds.

We note that with $n_{\mathrm{H}_{2}} \sim 1 \times 10^{5}$, a uniform density profile and spherical gas distribution the dynamical mass implies an upper limit on the dense gas volume filling factor of 0.002 within the central $600 \mathrm{pc}$ of NGC 6240 . This is consistent with the image, presented above, of the molecular disk being almost entirely filled with hot, shocked gas, but with the majority (by mass) of the molecular gas surviving in cold, dense clouds embedded within the hot phase.

Since the majority of the dense gas and the ongoing star formation are separate in NGC 6240 (Tacconi et al. 1999) we cannot calculate reliable local gas depletion times and instead we explore the galaxy averaged gas depletion time. Combining the star formation rate (SFR) of $60 \pm 30 M_{\odot} \mathrm{yr}^{-1}$ (Yun \& Carilli 2002; Feruglio et al. 2013) with our $\alpha_{\mathrm{HCN}(1-0)}$ and $L_{\mathrm{HCN}(1-0)}^{\prime}$ we can obtain estimates of the gas depletion time in NGC 6240, and we find a depletion time $6_{4}^{13} \times 10^{8} \mathrm{yr}$.

However, the dynamical mass in the central $600 \mathrm{pc}$ of $\sim 10^{10} M_{\odot}$ is significantly less than the dense gas mass implied by HCN and the dense phase CO. If we exclude results predicting gas masses in excess of the dynamical mass the depletion time is reduced to $0.9_{0.6}^{1.9} \times 10^{8} \mathrm{yr}$.

We do however question whether the dynamical mass is in fact particularly meaningful in the central regions of NGC 6240. This galaxy is an ongoing major merger, so it is not clear that the velocity dispersion should actually correlate with the mass. Estimating the mass from the velocity dispersion assumes either virialisation or stable orbital rotation, neither of which is necessarily true for 
the central gas in NGC 6240, which is dissipating turbulent energy from the merger (Tacconi et al. 1999) and is most likely a transient feature itself. It is very possible that this explains the super-dynamical masses suggested by some regions of our results and a large region of the parameter space in Papadopoulos et al. (2014).

\subsection{Implications of the $[\mathrm{HCN}] /\left[\mathrm{HCO}^{+}\right]$Abundance Ratio}

We have found $[\mathrm{HCN}] /\left[\mathrm{HCO}^{+}\right]=6.2_{4.3}^{7.9}$ in $\mathrm{NGC} 6240$. This compares favourably to the value of "around 10" found by Krips et al. (2008). NGC 6240 is frequently compared to Arp 220, where Tunnard et al. (2015) found $[\mathrm{HCN}] /\left[\mathrm{HCO}^{+}\right]>18$ in the western nucleus $(\mathrm{WN})$ and $[\mathrm{HCN}] /\left[\mathrm{HCO}^{+}\right] 0.5-5$ in the eastern nucleus $(\mathrm{EN})$. The abundance ratio for NGC 6240 appears to be most consistent with the less luminous EN, which is dominated by star formation (whereas Arp $220 \mathrm{WN}$ may contain an energetically significant AGN).

However, in NGC 6240 the comparison is probably less meaningful. Unlike in most of the galaxies of Krips et al. (2008), the majority of the molecular gas is spatially separated from the starbursts and putative AGNs in the two nuclei. As Krips et al. (2008) pointed out, the extreme conditions in NGC 6240 make it quite unique, and the abundance ratios may not carry the same meaning as they do in other galaxies and Arp 220 (which may itself be in a subset of galaxies possessing Compact Obscured Nuclei (CONs), which generate unique chemistries Costagliola et al. 2015). In the context of Krips et al. (2008), our results place NGC 6240 in the intermediate region between a starburst and AGN dominated galaxy. The derived $n_{\mathrm{H}_{2}}$ is higher than the usual $<10^{4.5} \mathrm{~cm}^{-3}$ for $\mathrm{AGNs}$, while the $[\mathrm{HCN}] /\left[\mathrm{HCO}^{+}\right]$ratio is higher than the $0.01-1$ of starburst but lower than the $>10$ of AGN. Given the separation of the starburst and AGN nuclei from the bulk of the molecular gas (which lies between the two nuclei) the fact that NGC 6240 does not fit clearly into any specific category is not surprising.

\section{CONCLUSIONS}

We have used new observations of the ${ }^{13} \mathrm{C}$ isotopologue lines of $\mathrm{HCN}$ and $\mathrm{HCO}^{+}$in NGC 6240 to model the physical conditions and abundance ratios of the molecular gas. We have:

- presented the first observations of $\mathrm{SiO}(2-1)$ and of the isotopologue line $\mathrm{H}^{13} \mathrm{CN}(1-0)$ in $\mathrm{NGC} 6240$, and an upper limit on $\mathrm{H}^{13} \mathrm{CO}^{+}(1-0)$.

- combined these observations with literature data as inputs for an MCMC wrapper for RADEX, modelling three gas phases with six species simultaneously to account for shocked molecular gas, extended diffuse molecular gas and cold, dense molecular gas, to find the conditions of the gas in NGC 6240. These models suggest that the cold, dense gas exists in multiple, small (0.01-0.1 pc) clouds embedded in the hot, shocked inter-nuclear gas disk. The cold gas is particularly cold with $T_{\mathrm{k}}=13_{10}^{20}$, while the hot phase reaches temperatures of almost $2000 \mathrm{~K}$.

- used our MCMC code and the new ${ }^{13} \mathrm{C}$ observations to demonstrate that the very high $\left[{ }^{12} \mathrm{C}\right] /\left[{ }^{13} \mathrm{C}\right]$ ratio in NGC 6240 found by Papadopoulos et al. (2014) was most likely due to assuming that the high $-J \mathrm{CO}$ lines originate in the dense gas traced by $\mathrm{HCN}$, whereas in NGC 6240 they originate in shocks (Meijerink et al. 2013) and the HCN lines are cold, dense gas dominated, isolated by using our new $\mathrm{H}^{13} \mathrm{CN}$ observations. Our models suggest instead that $\left[{ }^{12} \mathrm{C}\right] /\left[{ }^{13} \mathrm{C}\right]=98_{65}^{230}$ and is "standard" for ULIRGs, and even consistent with the Milky Way average value of 68 . The high $[\mathrm{HCN}] /\left[\mathrm{H}^{13} \mathrm{CN}\right]$ and $\left[\mathrm{HCO}^{+}\right] /\left[\mathrm{H}^{13} \mathrm{CO}^{+}\right]$ratios $\left(300_{200}^{500}\right)$ are due to isotope fractionation in cold, dense gas, with the $\left[\mathrm{HCO}^{+}\right] /\left[\mathrm{H}^{13} \mathrm{CO}^{+}\right]$ratio being particularly uncertain.

- derived $\alpha_{\mathrm{CO}}$ values for the diffuse, shocked and dense gas phases in NGC 6240, and combined these to produce a global $\alpha_{\mathrm{CO}}=1.5_{1.1}^{7.1}$, in between the ULIRG value of 0.8 and 4.2 appropriate for Milky Way GMCs. We also calculate $\alpha_{\mathrm{HCN}}=32_{13}^{89}$, where $\alpha_{\mathrm{HCN}}$ is tracing the cold, dense gas.

These results demonstrate the extreme value of isotopologue lines in LVG modelling, with their powerful ability to break degeneracies even when the isotopologue abundance ratios are unknown. Similarly, we have highlighted the dangers of fitting multiple species gas phase components by eye, as was quantified for multiple $\mathrm{CO}$ gas phases by Kamenetzky et al. (2014).

Our model produces chemically feasible molecular abundances and isotopologue abundance ratios, despite the significant simplifications. Nevertheless, the high $\left[\mathrm{HCO}^{+}\right] /\left[\mathrm{H}^{13} \mathrm{CO}^{+}\right]$ratio is unexpected, and warrants further investigation. A distinct possibility, evidenced by our slightly low continuum flux density, is that we are missing a significant fraction of the molecular line flux. This would present as elevated $[\mathrm{HCN}] /\left[\mathrm{H}^{13} \mathrm{CN}\right]$ and $\left[\mathrm{HCO}^{+}\right] /\left[\mathrm{H}^{13} \mathrm{CO}^{+}\right]$abundance ratios. Importantly, even if this is the case the main conclusions of this work, that the elemental $\left[{ }^{12} \mathrm{C}\right] /\left[{ }^{13} \mathrm{C}\right]$ ratio is not elevated in NGC 6240, are not affected; they are instead reinforced if we are missing line flux.

In any case, further observations of ${ }^{13} \mathrm{CO}, \mathrm{H}^{13} \mathrm{CN}$ and $\mathrm{H}^{13} \mathrm{CO}^{+}$are warranted, both in NGC 6240 and other galaxies with $\mathrm{CO}, \mathrm{HCN}$ and $\mathrm{HCO}^{+}$ SLEDs. These isotopologue lines provide added value and, as we have demonstrated here, even a single line can simultaneously improve temperature constraints while constraining the isotopologue abundance ratio, without the need to assume a, potentially erroneous, ratio.

Acknowledgements: RT would like to thank the organisers and sponsors of the ICIC Data Analysis Workshop. This research is supported by an STFC PhD studentship. TRG acknowledges support from an STFC Advanced Fellowship. SGB acknowledges support from Spanish grants AYA2010-15169 and AYA2012-32295 and from the Junta de Andalucia through TIC-114 and the Excellence Project P08TIC-03531. AU acknowledges support from Spanish grants 
AYA2012-32295 and FIS2012-32096. AF and SGB acknowledge support from Spanish MICIN program CONSOLIDER IMAGENIO 2010 under grant ASTROMOL (ref. CSD200900038). We thank the anonymous referee for their construc- tive comments, which greatly helped in improving the structure and readability of this paper. This work was based on observations carried out with the IRAM PdBI, supported by INSU/CNRS(France), MPG(Germany), and IGN(Spain).

\section{REFERENCES}

Aalto, S., Garcia-Burillo, S., Muller, S., et al. 2012, A\&A, 537, A44

Aalto, S., Polatidis, A. G., Hüttemeister, S., \& Curran, S. J. 2002, A\&A, 381, 783

Aalto, S., Spaans, M., Wiedner, M. C., \& Hüttemeister, S. 2007, A\&A, 464, 193

Aalto, S., Garcia-Burillo, S., Muller, S., et al. 2015, A\&A, 574, A85

Armus, L., Bernard-Salas, J., Spoon, H. W. W., et al. 2006, ApJ, 640, 204

Boger, G. I., \& Sternberg, A. 2005, ApJ, 632, 302

Bolatto, A. D., Wolfire, M., \& Leroy, A. K. 2013, ARA\&A, 51, 207

Costagliola, F., Sakamoto, K., Muller, S., et al. 2015, ArXiv e-prints, arXiv:1506.09027

Crane, P., \& Hegyi, D. J. 1988, ApJ, 326, L35

Crane, P., Hegyi, D. J., \& Lambert, D. L. 1991, ApJ, 378, 181

Dayou, F., \& Balança, C. 2006, A\&A, 459, 297

Dopita, M. A., Groves, B. A., Fischera, J., et al. 2005, ApJ, 619, 755

Downes, D., \& Solomon, P. M. 1998, ApJ, 507, 615

Dumouchel, F., Faure, A., \& Lique, F. 2010, MNRAS, 406, 2488

Elitzur, M., \& Watson, W. D. 1978, ApJ, 222, L141

—. 1980, ApJ, 236, 172

Feruglio, C., Fiore, F., Maiolino, R., et al. 2013, A\&A, 549, A51

Flower, D. R. 1999, MNRAS, 305, 651

Fuente, A., García-Burillo, S., Gerin, M., et al. 2005, ApJ, 619, L155

Fuente, A., Martin-Pintado, J., \& Gaume, R. 1995, ApJ, 442, L33

Gao, Y., \& Solomon, P. M. 2004, ApJS, 152, 63

García-Burillo, S., Usero, A., Alonso-Herrero, A., et al. 2012, A\&A, 539, A8

García-Burillo, S., Combes, F., Usero, A., et al. 2014, A\&A, 567, A 125

Goldsmith, P. F., \& Langer, W. D. 1999, ApJ, 517, 209

Graciá-Carpio, J., García-Burillo, S., Planesas, P., Fuente, A., \& Usero, A. 2008, A\&A, 479, 703

Greaves, J. S., \& Church, S. E. 1996, MNRAS, 283, 1179

Green, S., \& Thaddeus, P. 1974, ApJ, 191, 653

Greve, T. R., Papadopoulos, P. P., Gao, Y., \& Radford, S. J. E. 2009, ApJ, 692, 1432

Greve, T. R., Leonidaki, I., Xilouris, E. M., et al. 2014, ApJ, 794, 142

Hagiwara, Y., Baan, W. A., \& Klöckner, H.-R. 2011, AJ, 142, 17

Hastings, W. K. 1970, Biometrika, 57, 97

Henkel, C., Downes, D., Weiß, A., Riechers, D., \& Walter, F. 2010, A\&A, 516, A111

Henkel, C., Asiri, H., Ao, Y., et al. 2014, A\&A, 565, A3

Iono, D., Wilson, C. D., Takakuwa, S., et al. 2007, ApJ, 659, 283

Kamenetzky, J., Rangwala, N., Glenn, J., Maloney, P. R., \& Conley, A. 2014, ApJ, 795, 174

Komossa, S., Burwitz, V., Hasinger, G., et al. 2003, ApJ, 582, L15

Kramer, C., Alves, J., Lada, C. J., et al. 1999, A\&A, 342, 257

Krips, M., Neri, R., García-Burillo, S., et al. 2008, ApJ, 677, 262

Lada, E. A. 1992, ApJ, 393, L25

Lambert, D. L., Sheffer, Y., Gilliland, R. L., \& Federman, S. R. 1994, ApJ, 420, 756

Langer, W. D., Graedel, T. E., Frerking, M. A., \& Armentrout, P. B. 1984, ApJ, 277, 581

Langer, W. D., Wilson, R. W., Henry, P. S., \& Guelin, M. 1978, ApJ, 225, L139

Lavendy, H., Robbe, J. M., \& Gandara, G. 1987, Journal of Physics B Atomic Molecular Physics, 20, 3067

Lique, F., Spielfiedel, A., \& Cernicharo, J. 2006, A\&A, 451, 1125

Liu, T., Wu, Y., \& Zhang, H. 2013, ApJ, 775, L2

Lohr, L. L. 1998, J. Chem. Phys., 108, 8012

Loison, J.-C., Wakelam, V., \& Hickson, K. M. 2014, MNRAS, 443,398

Lutz, D., Sturm, E., Genzel, R., et al. 2003, A\&A, 409, 867
Martín, S., Kohno, K., Izumi, T., et al. 2015, A\&A, 573, A116

McMullin, J. P., Waters, B., Schiebel, D., Young, W., \& Golap, K. 2007, in Astronomical Society of the Pacific Conference Series, Vol. 376, Astronomical Data Analysis Software and Systems XVI, ed. R. A. Shaw, F. Hill, \& D. J. Bell, 127

Meijerink, R., Kristensen, L. E., Weiß, A., et al. 2013, ApJ, 762, L16

Metropolis, N., Rosenbluth, A. W., Rosenbluth, M. N., Teller, A. H., \& Teller, E. 1953, J. Chem. Phys., 21, 1087

Milam, S. N., Savage, C., Brewster, M. A., Ziurys, L. M., \& Wyckoff, S. 2005, ApJ, 634, 1126

Mladenović, M., \& Roueff, E. 2014, A\&A, 566, A144

Müller, H. S., Schlöder, F., Stutzki, J., \& Winnewisser, G. 2005 Journal of Molecular Structure, 742, 215

Nakajima, T., Takano, S., Kohno, K., \& Inoue, H. 2011, The Astrophysical Journal Letters, 728, L38

Nakanishi, K., Okumura, S. K., Kohno, K., Kawabe, R., \& Nakagawa, T. 2005, PASJ, 57, 575

Nguyen, Q.-R., Jackson, J. M., Henkel, C., Truong, B., \& Mauersberger, R. 1992, ApJ, 399, 521

Papadopoulos, P. P. 2007, ApJ, 656, 792

Papadopoulos, P. P., Isaak, K., \& van der Werf, P. 2010, ApJ, 711, 757

Papadopoulos, P. P., Röttgering, H. J. A., van der Werf, P. P., et al. 2000, ApJ, 528, 626

Papadopoulos, P. P., Thi, W.-F., Miniati, F., \& Viti, S. 2011, MNRAS, 414, 1705

Papadopoulos, P. P., van der Werf, P., Xilouris, E., Isaak, K. G., \& Gao, Y. 2012, ApJ, 751, 10

Papadopoulos, P. P., Zhang, Z.-Y., Xilouris, E. M., et al. 2014 ApJ, 788, 153

Pirogov, L., Zinchenko, I., Lapinov, A., Myshenko, V., \& Shul'Ga, V. 1995, Astronomy and Astrophysics Supplement Series, 109, 333

Remijan, A. J., Markwick-Kemper, A., \& ALMA Working Group on Spectral Line Frequencies. 2007, in Bulletin of the American Astronomical Society, Vol. 39, American Astronomical Society Meeting Abstracts, 132.11

Ripple, F., Heyer, M. H., Gutermuth, R., Snell, R. L., \& Brunt, C. M. 2013, MNRAS, 431, 1296

Ritchey, A. M., Federman, S. R., \& Lambert, D. L. 2011, ApJ, 728,36

Roueff, E., Loison, J. C., \& Hickson, K. M. 2015, A\&A, 576, A99

Sakamoto, K., Aalto, S., Evans, A. S., Wiedner, M. C., \& Wilner, D. J. 2010, ApJ, 725, L228

Sanders, D. B., Mazzarella, J. M., Kim, D.-C., Surace, J. A., \& Soifer, B. T. 2003, AJ, 126, 1607

Scoville, N., Sheth, K., Walter, F., et al. 2015, ApJ, 800, 70

Sheffer, Y., Rogers, M., Federman, S. R., Lambert, D. L., \& Gredel, R. 2007, ApJ, 667, 1002

Solomon, P. M., Downes, D., \& Radford, S. J. E. 1992, ApJ, 387, L55

Solomon, P. M., Rivolo, A. R., Barrett, J., \& Yahil, A. 1987, ApJ, 319,730

Szücs, L., Glover, S. C., \& Klessen, R. S. 2014, Monthly Notices of the Royal Astronomical Society, 445, 4055

Tacconi, L. J., Genzel, R., Tecza, M., et al. 1999, ApJ, 524, 732

Tafalla, M., Myers, P. C., Caselli, P., Walmsley, C. M., \& Comito, C. 2002, ApJ, 569,815

Tecza, M., Genzel, R., Tacconi, L. J., et al. 2000, ApJ, 537, 178

Tunnard, R., Greve, T. R., Garcia-Burillo, S., et al. 2015, ApJ, 800,25

Usero, A., García-Burillo, S., Fuente, A., Martín-Pintado, J., \& Rodríguez-Fernández, N. J. 2004, A\&A, 419, 897

van der Tak, F. F. S., Black, J. H., Schöier, F. L., Jansen, D. J., \& van Dishoeck, E. F. 2007, A\&A, 468, 627

van Dishoeck, E. F., \& Black, J. H. 1988, ApJ, 334, 771

Vázquez-Semadeni, E., Ballesteros-Paredes, J., \& Rodríguez, L. F. 1997, ApJ, 474, 292 
Véron-Cetty, M.-P., \& Véron, P. 2006, A\&A, 455, 773

Viti, S., Natarajan, S., \& Williams, D. A. 2002, MNRAS, 336, 797

Viti, S., García-Burillo, S., Fuente, A., et al. 2014, A\&A, 570, A28

Wang, J., Zhang, J., Shi, Y., \& Zhang, Z. 2013, ApJ, 778, L39

Wang, J., Zhang, Z., \& Shi, Y. 2011, MNRASL, 416, L21

Wang, J., Nardini, E., Fabbiano, G., et al. 2014, ApJ, 781, 55
Watson, W. D., Anicich, V. G., \& Huntress, Jr., W. T. 1976, ApJ, 205, L165

Wright, E. L. 2006, PASP, 118, 1711

Yang, B., Stancil, P. C., Balakrishnan, N., \& Forrey, R. C. 2010, ApJ, 718, 1062

Young, J. S., \& Scoville, N. 1982, ApJ, 258, 467

Yun, M. S., \& Carilli, C. L. 2002, ApJ, 568, 88

Zhang, Z.-Y., Henkel, C., Gao, Y., et al. 2014, A\&A, 568, A122

\section{APPENDIX}

\section{A. LVG MODELS}

We wrote an MCMC wrapper for the LVG code RADEX to allow a rapid and efficient exploration of high dimension parameter spaces and easy generation of posterior pdfs. The code allows us to fit multiple species simultaneously, as well as fitting multiple gas phases simultaneously. The code is written in Python, with the RADEX calculations in Fortran presenting the rate limiting step: $\gtrsim 99.8 \%$ of the runtime is RADEX IO and calculations.

We adopt a Metropolis-Hastings (MH) sampler for its simplicity and robustness. The likelihoods from the LVG models are generally simple, contiguous shapes, and as such are well suited to the MH algorithm. We do not include covariance terms between the new parameters when sampling: each is sampled from a 1D Gaussian distribution. We adopt a $\chi^{2}$ likelihood function:

$$
\mathcal{L} \propto \exp \left(-\chi^{2} / 2\right)
$$

Constraints on the parameter ranges are implemented using uniform priors in log-space. The MCMC is conducted in log-space, with values converted to linear space for input to RADEX. This provides simple scale invariance and prevents biasing towards high values of the parameters.

We use stock RADEX, and as such must be wary of non-convergences, bad fluxes and high optical depths. These are checked for when reading the RADEX results, and if identified the point is rejected and resampled. The alternative would be to treat this as a point of zero likelihood - test runs of these two showed no differences in the results. Since the MCMC naturally tends towards regions of greater likelihood the RADEX errors are not usually significant except for when using randomised initial positions. In the rare instance that the code becomes trapped in a region of errors new initial parameters are randomly generated.

\section{LVG Parameters}

Our aim was to extend the results of Papadopoulos et al. (2014) with our new data and an MCMC model. We therefore adopted a similar parameter space and $K_{v i r}$ constraints: $0.5<K_{v i r}<20$. See Table 3 for the three phase parameter ranges and Table 6 for the precursor model inputs.

While we model the abundance and velocity gradient $X_{\text {mol }}$ and $\mathrm{d} v / \mathrm{d} r$, RADEX requires as inputs the column density $N_{m o l}$ and line width $\Delta v$. As only the ratio $N_{\text {mol }} / \Delta v$ is relevant ${ }^{15}$, we fix $\Delta v=10 \mathrm{~km} \mathrm{~s}^{-1}$ and find $N_{\text {mol }}$ from:

$$
N_{\mathrm{mol}}=\frac{3.08 \times 10^{18} n_{\mathrm{H}_{2}} X_{\mathrm{mol}} \Delta v}{\mathrm{~d} v / \mathrm{d} r} .
$$

\section{B. CLOUD KINEMATIC SEPARATION}

Our MCMC LVG model fits line ratios, so while it is ideal for identifying the dominant conditions of the sampled gas phases it is poorly adapted for identifying the masses within each phase. A simple but inelegant solution would be to identify the CO line luminosity from each gas phase and then use a canonical $\alpha_{\mathrm{CO}}$ factor to estimate the mass of the phase. This is however extremely susceptible to uncertainties in the $\alpha_{\mathrm{CO}}$ factor. Instead, we derive an alternative, self-consistent, method, which is used to generate $\alpha_{\mathrm{CO}}$ factors for each gas phase and for the galaxy as a whole. We apply a physically motivated upper limit on $N_{\mathrm{H}_{2}}$, thereby applying a limit on $\Delta v$ per cloud.

A single gas cloud representing the galaxy is inadequate, since the column density of $\mathrm{H}_{2}$ can be written as:

$$
N_{\mathrm{H}_{2}}=\frac{3.08 \times 10^{18} n_{\mathrm{H}_{2}} \Delta v}{\mathrm{~d} v / \mathrm{d} r} .
$$

For the high density gas phase this leads to column densities in excess of $10^{26} \mathrm{~cm}^{-2}$ ! In reality, the dense gas is not in a single cloud but distributed across the galaxy in many, much smaller clouds, so that $\Delta v$ is determined by the large scale motion in the galaxy and does not correspond to the LVG $\Delta v$, which is the line width of the putative emitting cloud.

${ }^{15}$ For a given product $N_{\text {mol }} / \Delta v$, changing $\Delta v$ only changes the quoted flux, proportional to the change in $\Delta v$, which cancels in any ratios. The optical depths and brightness temperatures remain unchanged. 
We therefore separate the emission into multiple identical clouds to obtain more realistic estimates of the mass of the gas phases, without assuming cloud line widths. We start with the general statement that for a cloud of uniform density:

$$
M_{\text {cloud }}=\frac{4 \pi}{3}\left(\frac{\zeta \Delta v}{2 \mathrm{~d} v / \mathrm{d} r}\right)^{3} n_{\mathrm{H}_{2}} \mu_{\mathrm{H}_{2}}
$$

where $\zeta=3.08 \times 10^{18}$ is the parsec to cm conversion factor, $\Delta v$ is the FWHM line width of the cloud, $\mathrm{d} v / \mathrm{d} r$ is the velocity gradient of the cloud in $\mathrm{km} \mathrm{s}^{-1} \mathrm{pc}^{-1}, n_{\mathrm{H}_{2}}$ is the hydrogen number density in $\mathrm{cm}^{-3}$ and $\mu_{\mathrm{H}_{2}}$ is the helium adjusted ISM mass per $\mathrm{H}_{2}$ molecule.

Separately, we make the general statement of beam dilution that for a Gaussian surface brightness with peak $T_{\mathrm{b}}$ and a Gaussian beam much larger than the size of the cloud:

$$
T_{\mathrm{b}, \mathrm{d}}=\frac{T_{\mathrm{b}} \chi^{2} 1.133}{\Omega_{\mathrm{beam}}}\left(\frac{\Delta v}{2 \mathrm{~d} v / \mathrm{d} r}\right)^{2},
$$

where $\chi=\frac{1}{494} \operatorname{arcsec} / \mathrm{pc}$ is the angular scale for NGC 6240 and $\Omega_{\text {beam }}$ is in $\operatorname{arcsec}^{2}$. In this case, $T_{\mathrm{b}}$ is a result of RADEX runs, which give optically thick clouds for the HCN and CO $J=1-0$ lines.

For a Gaussian line, or a line which can be described as a combination of Gaussians, the number of clouds is the ratio of the area $(T \Delta v)$ of the observed line to the diluted area per cloud:

$$
n_{\text {clouds }}=\frac{T_{\mathrm{obs}} \mathrm{FWHM}}{T_{\mathrm{b}, \mathrm{d}} \Delta v}=\frac{T_{\mathrm{obs}} \Omega_{\mathrm{beam}} \mathrm{FWHM}}{1.133 T_{\mathrm{b}} \Delta v \chi^{2}\left(\frac{\Delta v}{2 \mathrm{~d} v / \mathrm{d} r}\right)^{2}} .
$$

Therefore the total mass in the galaxy in this gas phase is:

$$
\begin{aligned}
M & =M_{\text {cloud }} n_{\text {clouds }} \\
& =\frac{4 \pi}{3}\left(\frac{\zeta \Delta v}{2 \mathrm{~d} v / \mathrm{d} r}\right)^{3} n_{\mathrm{H}_{2}} \mu_{\mathrm{H}_{2}} \frac{T_{\text {obs }} \Omega_{\text {beam }} \text { FWHM }}{1.133 T_{\mathrm{b}} \Delta v \chi^{2}\left(\frac{\Delta v}{2 \mathrm{~d} v / \mathrm{d} r}\right)^{2}} \\
& =\frac{1.85 \zeta^{3} \mu_{\mathrm{H}_{2}}}{\chi^{2}} \frac{T_{\text {obs }} \Omega_{\text {beam }}}{T_{\mathrm{b}}} \frac{n_{\mathrm{H}_{2}}}{\mathrm{~d} v / \mathrm{d} r} \mathrm{FWHM} \\
& =0.123 \frac{T_{\mathrm{obs}} \Omega_{\text {beam }}}{T_{\mathrm{b}} \chi^{2}} \frac{n_{\mathrm{H}_{2}}}{\mathrm{~d} v / \mathrm{d} r} \mathrm{FWHM} \quad\left[M_{\odot}\right]
\end{aligned}
$$

This in fact corresponds to the same equation for $\alpha_{\text {mol }}$ as that derived by Papadopoulos et al. (2012) by considering iso-velocity surfaces across a galaxy. Also, we find that the mass is independent of the chosen upper limit on $N_{\mathrm{H}_{2}}$ which is reassuring given that GMCs are likely to present a range of column densities (Vázquez-Semadeni et al. 1997).

\section{C: PRECURSOR LVG MODELS}

The three phase model presented in Section 4.1 makes assumptions regarding the gas phases and distribution of molecular species between phases. These assumptions are based on a series of models of increasing complexity that are precursors to our three phase, six species model. These models do not add to the scientific results of the paper, but due to their role in justifying our assumptions we include them here.

\section{Single Phase}

We first ran four models with single species and their respective isotopologues: $\mathrm{HCN}+\mathrm{H}^{13} \mathrm{CN}$ with the detected $\mathrm{H}^{13} \mathrm{CN}(1-0)$ line flux, $\mathrm{HCN}+\mathrm{H}^{13} \mathrm{CN}$ with the upper limit on the $\mathrm{H}^{13} \mathrm{CN}(1-0)$ line flux, $\mathrm{HCO}^{+}+\mathrm{H}^{13} \mathrm{CO}^{+}$and $\mathrm{CS}$ only, with CS data shown in Table 5. We also run a combined model with $\mathrm{HCN}+\mathrm{HCO}^{+}+\mathrm{H}^{13} \mathrm{CN}+\mathrm{H}^{13} \mathrm{CO}^{+}$. The input parameter ranges for these models are shown in Table 6. When fixed, molecular abundances were chosen to match those of Papadopoulos et al. (2014), i.e., $X_{\mathrm{HCN}}=2 \times 10^{-8}, X_{\mathrm{HCO}^{+}}=8 \times 10^{-9}$ and $X_{\mathrm{CS}}=1 \times 10^{-9}$.

The numerical results of the models are given in Table 7 , where we present both the the means of the MCMC traces and the best fit values. We recover similar temperatures and densities to Papadopoulos et al. (2014), although the inclusion of our new ${ }^{13} \mathrm{C}$ isotopologue lines allows us to place tighter constraints on these parameters. 
TABLE 5

LVG CS LINE INPUTS

\begin{tabular}{ccccc}
\cline { 2 - 4 } Line & $\begin{array}{c}\nu_{0} \\
{[\mathrm{GHz}]}\end{array}$ & $\begin{array}{c}S_{\text {line }} \\
{\left[\mathrm{Jy} \mathrm{km} \mathrm{s}{ }^{-1}\right]}\end{array}$ & References $^{\mathrm{a}}$ \\
\hline $\mathrm{CS}(2-1)$ & 97.98 & $7.5 \pm 1.5$ & 1 \\
$\mathrm{CS}(3-2)$ & 145.0 & $9 \pm 2$ & 1 \\
$\mathrm{CS}(5-4)$ & 239.1 & $54 \pm 6$ & 2 \\
$\mathrm{CS}(7-6)$ & 342.9 & $7.4 \pm 1.0$ & 3 \\
\hline
\end{tabular}

${ }^{\mathrm{a}} 1$ = Papadopoulos et al. (2014), 2 = Wang et al. (2011), 3 = Scoville et al. (2015)

TABLE 6

MCMC MODEL INPUT PARAMETER RANGES.

\begin{tabular}{|c|c|c|c|c|c|c|c|c|c|}
\hline Model & ID & $\begin{array}{l}T_{\mathrm{k}} \\
{[\mathrm{K}]}\end{array}$ & $\begin{array}{c}n_{\mathrm{H}_{2}} \\
{\left[\mathrm{~cm}^{-3}\right]}\end{array}$ & $\begin{array}{c}\mathrm{d} v / \mathrm{d} r \\
{\left[\mathrm{~km} \mathrm{~s}^{-1} \mathrm{pc}^{-1}\right]}\end{array}$ & $\begin{array}{l}\text { All values lo } \\
\frac{\left[\mathrm{HC}\left(\mathrm{N} / \mathrm{O}^{+}\right)\right]}{\left[\mathrm{H}^{13} \mathrm{C}\left(\mathrm{N} / \mathrm{O}^{+}\right)\right]}\end{array}$ & $X_{\mathrm{HCN}}$ & $X_{\mathrm{SiO}}$ & $X_{\mathrm{HCO}^{+}}$ & $X_{\mathrm{CS}}$ \\
\hline HCN \& H $\mathrm{H}^{13} \mathrm{CN}$, measured & $1 \mathrm{1a}$ & $0.5-3$ & $2-8$ & $0-3$ & 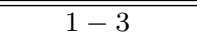 & -7.70 & ב... & 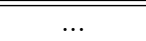 & $\overline{c . \ldots}$ \\
\hline $\mathrm{HCN} \& \mathrm{H}^{13} \mathrm{CN}$, upper limit ..... & $1 \mathrm{~b}$ & $0.5-3$ & $2-8$ & $0-3$ & $1-3$ & -7.70 & $\ldots$ & $\ldots$ & $\ldots$ \\
\hline $\mathrm{HCO}^{+} \& \mathrm{H}^{13} \mathrm{CO}^{+} \ldots \ldots \ldots \ldots$ & 2 & $0.5-3$ & $2-8$ & $0-3$ & $1-3$ & $\ldots$ & $\ldots$ & -8.10 & $\ldots$ \\
\hline $\mathrm{CS}$ & 5 & $0.5-3$ & $2-8$ & $0-3$ & $\ldots$ & $\ldots$ & $\ldots$ & $\ldots$ & -9.00 \\
\hline $\mathrm{HCN}, \mathrm{HCO}^{+}, \mathrm{H}^{13} \mathrm{CN} \& \mathrm{H}^{13} \mathrm{CO}^{+}$ & 4 & $0.5-3$ & $2-8$ & $0-3$ & $1-3$ & $-12--4$ & $\ldots$ & $-12--4$ & $\ldots$ \\
\hline
\end{tabular}

TABLE 7

MCMC RESULTS

Posterior mean results and the upper and lower limits on the $68 \%$ credible interval.

\begin{tabular}{|c|c|c|c|c|c|c|c|c|}
\hline Model & ID & $\begin{array}{l}T_{\mathrm{k}} \\
{[\mathrm{K}]}\end{array}$ & $\begin{array}{c}n_{\mathrm{H}_{2}} \\
{\left[\mathrm{~cm}^{-3}\right]}\end{array}$ & $\begin{array}{c}\mathrm{d} v / \mathrm{d} r \\
{\left[\mathrm{~km} \mathrm{~s}^{-1} \mathrm{pc}^{-1}\right]}\end{array}$ & $\begin{array}{l}\text { All values } \log _{10} \\
\frac{\left[\mathrm{HC}\left(\mathrm{N} / \mathrm{O}^{+}\right)\right]}{\left[\mathrm{H}^{13} \mathrm{C}\left(\mathrm{N} / \mathrm{O}^{+}\right)\right]}\end{array}$ & $X_{\mathrm{HCN}}$ & $X_{\mathrm{SiO}}$ & $X_{\mathrm{HCO}^{+}}$ \\
\hline "HCN \& $\mathrm{H}^{13} \mathrm{CN}$, measured $\ldots . .$. & $1 \mathrm{a}$ & $1.52_{1.00}^{1.75}$ & $4.93_{4.40}^{5.60}$ & $1.28_{0.42}^{2.28}$ & $2.27_{1.90}^{2.46}$ & 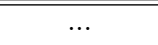 & 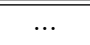 & 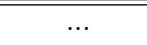 \\
\hline $\mathrm{HCN} \& \mathrm{H}^{13} \mathrm{CN}$, upper limit $\ldots$. & $1 b$ & $1.50_{0.95}^{1.75}$ & $4.95_{4.22}^{5.84}$ & $1.39_{0.42}^{1.98}$ & $2.49_{2.34}^{3.00}$ & $\ldots$ & $\ldots$ & $\ldots$ \\
\hline $\mathrm{HCO}^{+} \& \mathrm{H}^{13} \mathrm{CO}^{+} \ldots \ldots \ldots \ldots$ & 2 & $1.39_{1.13}^{1.60}$ & $4.51_{3.86}^{4.82}$ & $1.00_{0.03}^{1.05}$ & $2.74_{2.68}^{3.00}$ & $\ldots$ & $\ldots$ & $\ldots$ \\
\hline $\mathrm{CS} \ldots \ldots \ldots \ldots \ldots \ldots \ldots \ldots \ldots$ & 3 & $2.71_{2.65}^{3.0}$ & $3.3_{3.0}^{4.1}$ & $0.68_{0.03}^{0.93}$ & $\cdots$ & $\ldots$ & $\ldots$ & $\ldots$ \\
\hline $\mathrm{HCN}, \mathrm{HCO}^{+}, \mathrm{H}^{13} \mathrm{CN} \& \mathrm{H}^{13} \mathrm{CO}^{+}$ & 4 & $1.04_{0.85}^{1.13}$ & $5.87_{5.42}^{6.62}$ & $1.81_{1.26}^{2.34}$ & $2.61_{2.42}^{2.82}$ & $-8.32_{-9.12}^{-7.52}$ & $\ldots$ & $-9.04_{-9.84}^{-8.4}$ \\
\hline
\end{tabular}

Joint maximum likelihood results.

\begin{tabular}{|c|c|c|c|c|c|c|c|c|}
\hline Model & ID & $\begin{array}{l}T_{\mathrm{k}} \\
{[\mathrm{K}]}\end{array}$ & $\begin{array}{c}n_{\mathrm{H}_{2}} \\
{\left[\mathrm{~cm}^{-3}\right]} \\
\end{array}$ & $\begin{array}{c}\mathrm{d} v / \mathrm{d} r \\
{\left[\mathrm{~km} \mathrm{~s}^{-1} \mathrm{pc}^{-1}\right]}\end{array}$ & $\begin{array}{l}\frac{\left[\mathrm{HC}\left(\mathrm{N} / \mathrm{O}^{+}\right)\right]}{\left[\mathrm{H}^{13} \mathrm{C}\left(\mathrm{N} / \mathrm{O}^{+}\right)\right]}\end{array}$ & $X_{\mathrm{HCN}}$ & $X_{\mathrm{SiO}}$ & $X_{\mathrm{HCO}^{+}}$ \\
\hline HCN \& $\mathrm{H}^{13} \mathrm{CN}$, measured $\ldots . .$. & 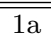 & 0.86 & 6.3 & 2.7 & 3.0 & 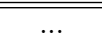 & 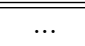 & ב... \\
\hline $\mathrm{HCN} \& \mathrm{H}^{13} \mathrm{CN}$, upper limit $\ldots .$. & $1 \mathrm{~b}$ & 0.88 & 6.3 & 2.7 & 3.0 & $\ldots$ & $\ldots$ & $\ldots$ \\
\hline $\mathrm{HCO}^{+} \& \mathrm{H}^{13} \mathrm{CO}^{+} \ldots \ldots \ldots \ldots \ldots$ & 2 & 1.1 & 4.8 & 1.1 & 3.0 & $\ldots$ & $\ldots$ & $\ldots$ \\
\hline $\mathrm{CS} \ldots \ldots \ldots \ldots \ldots \ldots \ldots$ & 3 & 3.0 & 3.5 & 0.1 & $\ldots$ & $\ldots$ & $\ldots$ & $\ldots$ \\
\hline $\mathrm{HCN}, \mathrm{HCO}^{+}, \mathrm{H}^{13} \mathrm{CN} \& \mathrm{H}^{13} \mathrm{CO}^{+}$ & 4 & 0.96 & 6.3 & 1.7 & 2.6 & -9.0 & $\ldots$ & -9.8 \\
\hline
\end{tabular}

The Effect of $T_{\mathrm{bg}}$

For some very active galaxies with luminous dust emission, background radiation from hot dust behind the gas cloud may significantly affect the line ratios (Papadopoulos et al. 2010). For dense molecular gas tracers, neglecting this may lead to extremely unusual solutions from RADEX fits to the line ratios.

We explored this possibility in NGC 6240 by testing whether increasing $T_{\mathrm{bg}}$ can improve the fits of our HCN + $\mathrm{HCO}^{+}$single phase model. The model was rerun with $T_{\mathrm{bg}}=10,20$ and $50 \mathrm{~K}$.

We find that increasing $T_{\mathrm{bg}}$ to $10 \mathrm{~K}$ marginally improves the $\mathrm{HCO}^{+}$SLED fit at the expense of a sightly worse $\mathrm{HCN}$ SLED fit. The solutions are constrained to give positive fluxes which indirectly constrains the gas kinetic temperature in these models to be $\gtrsim T_{\mathrm{bg}}$. The results push against this limit, so that $T_{\mathrm{k}} / T_{\mathrm{dust}} \sim T_{\mathrm{k}} / T_{\mathrm{bg}} \simeq 1$, which is unlikely to be reasonable in the extensively shocked environment of NGC 6240 (Meijerink et al. 2013; Papadopoulos et al. 2014), unless the $\mathrm{HCN}$ and $\mathrm{HCO}^{+}$are located not in shocks but in cold, dense cores. As $T_{\mathrm{bg}}$ is increased, the fits become progressively worse, with $50 \mathrm{~K}$ not remotely fitting the SLED and producing $\chi^{2}>1300$ (cf. $4-6$ for $T_{\text {bg }}=3-10 \mathrm{~K}$ ). The SLED fits for these models are shown in Figure 13. Note that the change in shape is largely due to a suppression of the low $-J$ line emission in excess of the background due to the increased background.

The $50 \mathrm{~K}$ case is comparable to the the $52 \mathrm{~K}$ black body dust temperature described by Tacconi et al. (1999). However, we found no consistent solutions over multiple runs, and pathological fits to the SLEDs for this $T_{\mathrm{bg}}$. This 

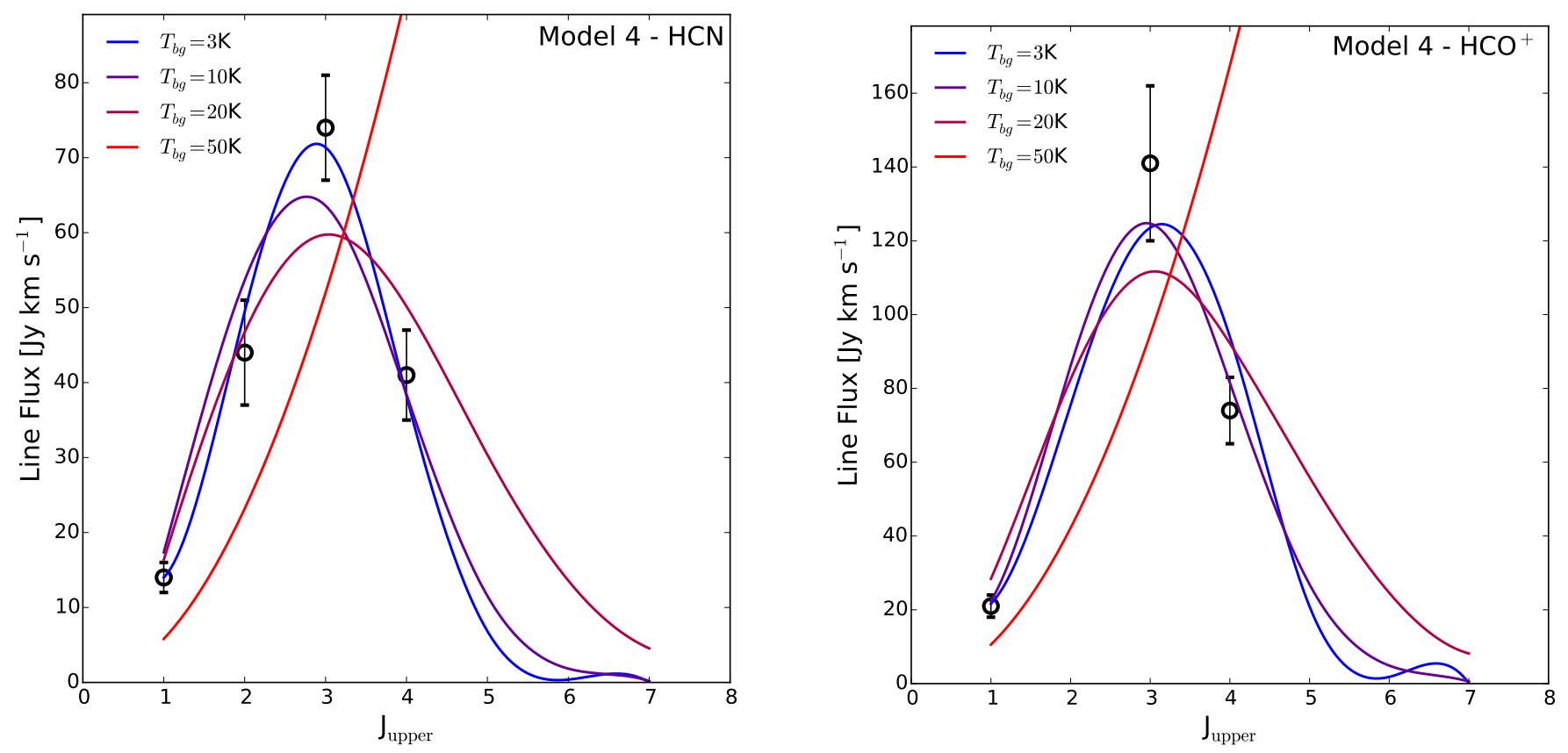

FIG. 13. - SLED fits for the $\mathrm{HCN}+\mathrm{HCO}^{+}$single phase model, with $T_{\mathrm{bg}}$ increasing as $3,10,20$ and $50 \mathrm{~K}$. The best fits are from the $3 \mathrm{~K}$ background, and the two observationally motivated background temperatures $(20$ and $50 \mathrm{~K})$ both produce much worse fits.

is consistent with the inconsistencies between the $\mathrm{CO}$ and $1.3 \mathrm{~mm}$ continuum estimates of gas mass commented on by Tacconi et al. (1999): they find that the dust co-located with the dense gas was most likely significantly cooler (perhaps 20-25 K), and furthermore that the shock heated gas is thermally decoupled from the cooler dust.

The inability of our results to find good a solution for a $50 \mathrm{~K}$ background is unsurprising, but the poor solution at $20 \mathrm{~K}$ is slightly more interesting. One possible, and we would argue likely, explanation could simply be that the HCN and $\mathrm{HCO}^{+}$emission are tracing the relatively quiescent regions of shielded, dense gas, i.e., they are performing as classical dense gas tracers. This is consistent with the marginal improvement (worsening) of the SLED fit of HCO ${ }^{+}$ ( $\mathrm{HCN}$ ) when $T_{\mathrm{bg}}$ is increased from $3 \mathrm{~K}$ to $10 \mathrm{~K}$, as $\mathrm{HCO}^{+}$is probably more extended and less shielded than $\mathrm{HCN}$, so subject to a slightly stronger background radiation field. It is also consistent with the very high $\mathrm{HCN} / \mathrm{CN}$ line ratio of 2 found by Aalto et al. (2002), which is evidence of large reservoirs of UV-shielded gas (Fuente et al. 1995; Greaves \& Church 1996).

We conclude that there is insufficient evidence to support using a super-CMB background and proceed with the $3 \mathrm{~K}$ CMB.

\section{Two Phase Modelling - $\mathrm{HCN}+\mathrm{HCO}^{+}$}

Here, we extend our model to include a second, non-interacting gas phase with both phases containing HCN and $\mathrm{HCO}^{+}$. This is strongly physically motivated as single dish observations across the galaxy are sampling multiple non-interacting gas phases confused within the beam.

Each of the two phases has their own kinetic temperature, density, and velocity gradient while they share common $\mathrm{HCN}$ and $\mathrm{HCO}^{+}$abundances and a single $\left[\mathrm{HC}\left(\mathrm{N} / \mathrm{O}^{+}\right)\right] /\left[\mathrm{H}^{13} \mathrm{C}\left(\mathrm{N} / \mathrm{O}^{+}\right)\right]$abundance ratio ${ }^{16}$. The fluxes from the two phases are added, weighted by a free parameter, $f$, such that:

$$
S=S_{1} f+S_{2}(1-f)
$$

where $S_{1}$ and $S_{2}$ are the RADEX fluxes for phase 1 and 2 respectively, in a simplified version of the method used in (Zhang et al. 2014). Either of $S_{1}$ and $S_{2}$ may be negative, but the combined fluxes $S$ are constrained to be positive, as per observations. Furthermore, there is no line transfer between the two phases: they emulate two phases confused in the imaging beam, not two phases along the same line of sight (unless they are decoupled by a sufficiently large velocity offset).

The results of this two phase model are shown in Figure 14. It appears from Figure 14 that we have an underdetermined model unable to either constrain both phases or clearly exclude one. Even though we are unable to constrain the kinetic temperature, density or velocity gradient, the abundance ratio is well determined.

The $\left[\mathrm{HC}\left(\mathrm{N} / \mathrm{O}^{+}\right)\right] /\left[\mathrm{H}^{13} \mathrm{C}\left(\mathrm{N} / \mathrm{O}^{+}\right)\right]$ratio in this model is elevated. It is almost definitely unsuitable to have a single $\left[\mathrm{HC}\left(\mathrm{N} / \mathrm{O}^{+}\right)\right] /\left[\mathrm{H}^{13} \mathrm{C}\left(\mathrm{N} / \mathrm{O}^{+}\right)\right]$ratio for both phases and both $\mathrm{HCN}$ and $\mathrm{HCO}^{+}$; unfortunately, as is shown by the

\footnotetext{
16 While this is undesirable it is necessary to minimise the number of free parameters we attempt to constrain with our few line
} 


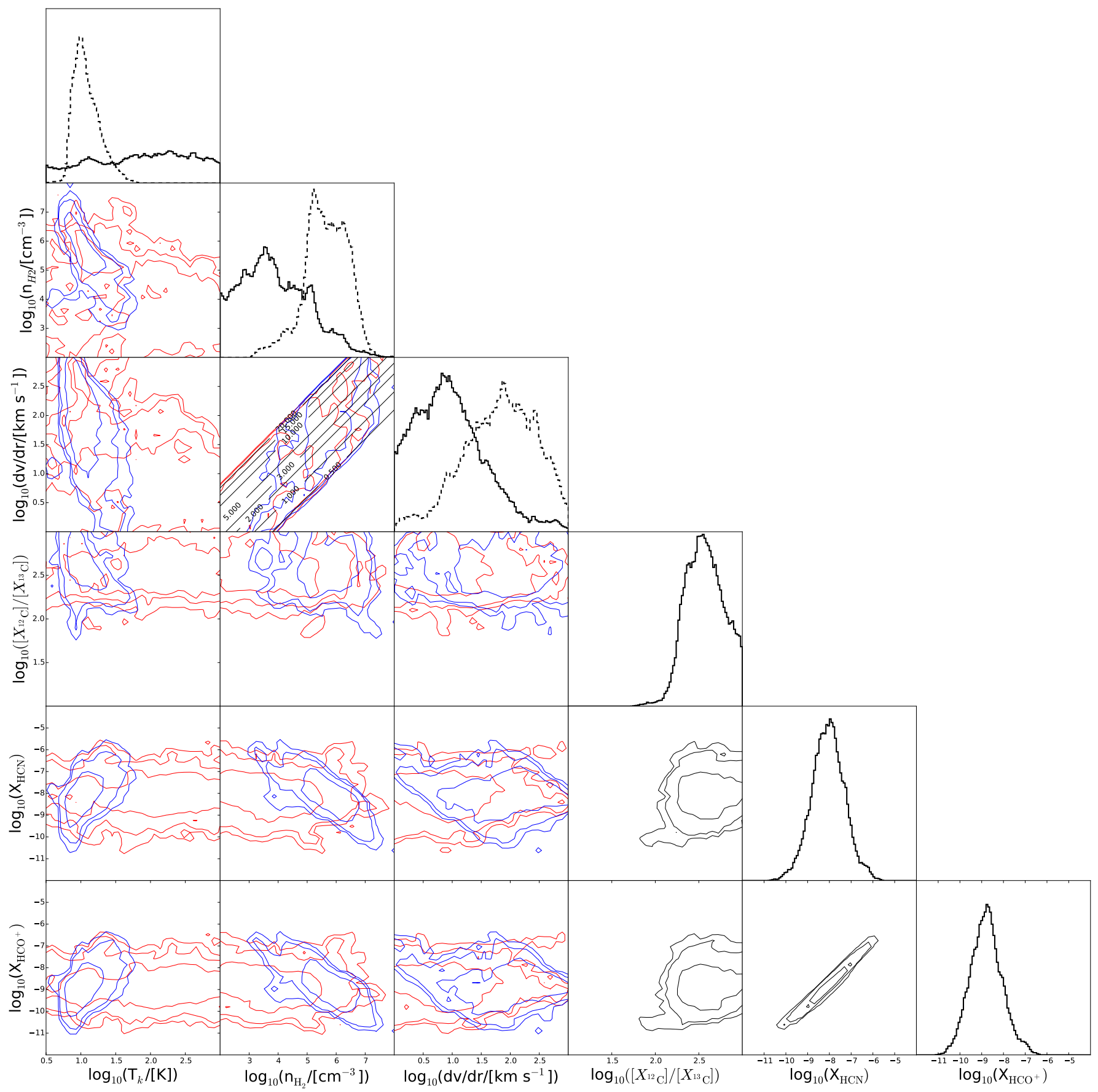

FIG. 14. - The modified step plot for the two phase $\mathrm{HCN}+\mathrm{HCO}^{+}$model. Contours are at the $68 \%, 95 \%$ and $99 \%$ credible intervals. Red/solid and blue/dashed contours are phases 1 and 2 , with $T_{\mathrm{bg}}=3$ and $3 \mathrm{~K}$ respectively. $\left[{ }^{12} \mathrm{C}\right] /\left[{ }^{13} \mathrm{C}\right], X_{\mathrm{HCN}}$ and $X_{\mathrm{HCO}}+$ are kept the same in the two phases, so have only one set of black contours. The two phases are completely degenerate - unsurprising given the good fits of the single phase models.

degeneracy of the two phases, we do not have sufficient observations nor are observations of sufficient precision to constrain two phases without making some simplifications. Further observations of the $\mathrm{H}^{13} \mathrm{CN}$ and $\mathrm{H}^{13} \mathrm{CO}{ }^{+}$SLEDs, in particular the $J=3-2$ line, would go a long way towards solving this issue.

\section{Two Phase Modelling - $\mathrm{HCN}+\mathrm{HCO}^{+}+\mathrm{CO}$}

The previous two phase model failed due to the paucity of available molecular lines and the large uncertainties on those available. In lieu of additional ${ }^{13} \mathrm{C}$ isotopologue line observations, which would place much tighter constraints on the models, we explore the addition of $\mathrm{CO}$ and ${ }^{13} \mathrm{CO}$ to the two phase models. We take the line fluxes directly from Papadopoulos et al. (2014), and do not make any corrections for source size: the $f$ scaling parameter allows the model to account for different beam filling factors due to the different sizes of the $\mathrm{HCN}$ and $\mathrm{CO}$ emitting regions, while the two phases allow for non-cospatial emission. 
TABLE 8

$\mathrm{HCN}+\mathrm{HCO}^{+}+\mathrm{CO}$ Two Phase Model Solutions. All values $\log _{10}$.

\begin{tabular}{|c|c|c|}
\hline Parameter & Mean & $\min \chi^{2}$ \\
\hline$\overline{T_{\mathrm{k}, 1} \ldots \ldots \ldots}$ & $3.2_{3.1}^{3.3}$ & 3.2 \\
\hline$T_{\mathrm{k}, 2}$ & $0.9_{0.8}^{0.9}$ & 0.8 \\
\hline$n_{\mathrm{H}_{2}, 1} \ldots \ldots \ldots \ldots \ldots \ldots \ldots \ldots \ldots \ldots \ldots \ldots$ & $3.6_{3.5}^{3.8}$ & 3.5 \\
\hline$n_{\mathrm{H}_{2}, 2}$ & $6.6_{6.3}^{6.8}$ & 6.5 \\
\hline$(\mathrm{d} v / \mathrm{d} r)_{1} \ldots$ & $0.8_{1.1}^{1.5}$ & 1.2 \\
\hline$(\mathrm{d} v / \mathrm{d} r)_{2} \ldots \ldots$ & $2.4_{2.3}^{3.0}$ & 2.9 \\
\hline$\left(\frac{[\mathrm{HCN}]}{\left[\mathrm{H}^{13} \mathrm{CN}\right]}=\frac{\left[\mathrm{HCO}^{+}\right]}{\left[\mathrm{H}^{13} \mathrm{CO}^{+}\right]}=\frac{[\mathrm{CO}]}{\left[{ }^{13} \mathrm{CO}\right]}\right)_{1}$ & $1.8_{1.7}^{2.0}$ & 1.9 \\
\hline$\left(\frac{[\mathrm{HCN}]}{\left[\mathrm{H}^{13} \mathrm{CN}\right]}=\frac{\left[\mathrm{HCO}^{+}\right]}{\left[\mathrm{H}^{13} \mathrm{CO}^{+}\right]}=\frac{[\mathrm{CO}]}{\left[{ }^{13} \mathrm{CO}\right]}\right)_{2}$ & $2.7_{2.7}^{3.0}$ & 2.9 \\
\hline$X_{\mathrm{HCN}, 1} \ldots \ldots \ldots \ldots \ldots \ldots \ldots \ldots$ & $-10.8_{-11.9}^{-10.4}$ & -10.0 \\
\hline$X_{\mathrm{HCN}, 2} \cdots$ & $-8.6_{-9.0}^{-7.9}$ & -7.7 \\
\hline$X_{\mathrm{HCO}}+, 1 \ldots \ldots \ldots \ldots \ldots \ldots$ & $-9.3_{-10.6}^{-6.7}$ & -6.6 \\
\hline$X_{\mathrm{HCO}+, 2} \cdots$ & $-9.3_{-9.6}^{-8.7}$ & -8.6 \\
\hline$X_{\mathrm{CO}, 1} \ldots \ldots \ldots \ldots \ldots \ldots \ldots \ldots$ & $-5.3_{-6.0}^{-4.5}$ & -4.4 \\
\hline$X_{\mathrm{CO}, 2}$. & $-6.1_{-6.3}^{-5.4}$ & -5.2 \\
\hline$f \ldots \ldots$ & $-0.9_{-1.2}^{-0.7}$ & -1.2 \\
\hline
\end{tabular}

With the additional lines we are able to extend the model complexity so that the free parameters include $X_{\mathrm{CO}}$, $X_{\mathrm{HCN}}, X_{\mathrm{HCO}^{+}}$and $\left[X_{{ }_{12} \mathrm{C}}\right] /\left[X_{{ }_{13} \mathrm{C}}\right]$ in each gas phase. Once again, we have insufficient lines to simultaneously fit free $X_{\mathrm{mol}}$ and $\left[X_{12} \mathrm{C}\right] /\left[X_{{ }^{13} \mathrm{C}}\right]$ ratios for every species in both phases, and we use a single $\left[X_{12} \mathrm{C}\right] /\left[X_{13} \mathrm{C}\right]$ ratio in each phase for all species. Even with these restrictions the code can take many more steps to converge, and the traces were carefully inspected to exclude unstable/unconverged runs. Of the runs that converged, all converged onto the same locus, which is presented here. The results are tabulated in Table 8 and shown in Figures 15 (parameter pdfs) and 16 (SLEDs).

This model provides very tight constraints on the two gas phases, although the tightness of the constraints is no guarantee of accuracy. The model spontaneously separates into a hot, diffuse, CO dominated phase and a cold, dense, HCN dominated phase. This result is robust to randomised initial positions over multiple MCMC runs and is similar to the findings of Kamenetzky et al. (2014) who used only CO. $T_{\mathrm{k}}$ and $n_{\mathrm{H}_{2}}$ are the best constrained parameters, with less than \pm 0.1 dex spread in the $68 \%$ credible intervals. In the diffuse phase $X_{\mathrm{HCN}}$ falls rapidly, and Figure 16 confirms that $\mathrm{HCN}$ has no contribution from the diffuse gas phase, so that $X_{\mathrm{HCN}}$ in this phase is an upper limit only. In both phases $X_{\mathrm{CO}}$ is slightly lower than the canonical value of $2 \times 10^{-4}$ presenting instead $4 \times 10^{-5}$ and $6 \times 10^{-6}$. However, the spread in $X_{\mathrm{CO}}$ is very large, especially in the hot phase.

\section{The $\left[X_{12} \mathrm{C}\right] /\left[X_{13} \mathrm{C}\right]$ ratio: evidence for fractionation?}

Even with the addition of the $\mathrm{CO}$ and ${ }^{13} \mathrm{CO}$ lines we are unable to constrain two gas phases with free $\mathrm{CO}$, $\mathrm{HCN}$ and $\mathrm{HCO}^{+} ;{ }^{13} \mathrm{CO}, \mathrm{H}^{13} \mathrm{CN}$ and $\mathrm{H}^{13} \mathrm{CO}^{+}$abundances in each phase. In the quest to identify whether the $\left[{ }^{12} \mathrm{C}\right] /\left[{ }^{13} \mathrm{C}\right]$ ratio found by Papadopoulos et al. (2014) is real, or due to biased SLED fitting, we ran a model with $X_{\mathrm{CO}}, X_{\mathrm{HCN}}$ and $X_{\mathrm{HCO}^{+}}$fixed to the best fit parameters of the previous run (see Table 8 for values). We then introduce as free parameters $[\mathrm{CO}] /\left[{ }^{13} \mathrm{CO}\right]_{1},[\mathrm{CO}] /\left[{ }^{13} \mathrm{CO}\right]_{2},[\mathrm{HCN}] /\left[\mathrm{H}^{13} \mathrm{CN}\right]$ and $\left[\mathrm{HCO}^{+}\right] /\left[\mathrm{H}^{13} \mathrm{CO}^{+}\right]$. I.e., while $\mathrm{HCN}$ and $\mathrm{HCO}^{+}$are present in both phases, each has a single isotopologue ratio used for both phases. This is motivated by us only having the $J=1-0$ line for the $\mathrm{H}^{13} \mathrm{CN}$ and $\mathrm{H}^{13} \mathrm{CO}^{+}$, which have negligible contributions in the hot phase - in effect we are indirectly excluding $\mathrm{H}^{13} \mathrm{CN}$ and $\mathrm{H}^{13} \mathrm{CO}^{+}$from the hot phase.

The results of the $\left[{ }^{12} \mathrm{C}\right] /\left[{ }^{13} \mathrm{C}\right]$ investigation model are shown in Figure 17 and Table 9. The model recovers very similar $T_{\mathrm{k}}, n_{\mathrm{H}_{2}}$ and $\mathrm{d} v / \mathrm{d} r$ as the previous model. As we have fixed the main isotope abundances this is not surprising, but it is an important confirmation that the isotopologue abundance ratios we derive here are compatible with the less restricted two phase model; by fixing some parameters and freeing others we have not inadvertently moved into a very different locus in parameter space.

The $[\mathrm{HCN}] /\left[\mathrm{H}^{13} \mathrm{CN}\right]$ and $\left[\mathrm{HCO}^{+}\right] /\left[\mathrm{H}^{13} \mathrm{CO}^{+}\right]$ratios are both very high, while the $[\mathrm{CO}] /\left[{ }^{13} \mathrm{CO}\right]$ ratio is $\sim 100-200$ in both phases. However, the abundance ratios are correlated, so that the spread in $\left([\mathrm{CO}] /\left[{ }^{13} \mathrm{CO}\right]_{2}\right) /\left([\mathrm{HCN}] /\left[\mathrm{H}^{13} \mathrm{CN}\right]\right)$ is extremely small, strongly peaking at $2-3$, consistent with Roueff et al. (2015). These results should be interpreted with caution: the fixing of the molecular abundances could be biasing the results. However, it is expected to bias them towards the values found in the previous model, so $[\mathrm{CO}] /\left[{ }^{13} \mathrm{CO}\right]_{2}$ lying much lower, while $[\mathrm{HCN}] /\left[\mathrm{H}^{13} \mathrm{CN}\right]$ and $\left[\mathrm{HCO}^{+}\right] /\left[\mathrm{H}^{13} \mathrm{CO}^{+}\right]$both lie higher, suggests that this is a real effect. This is evidence for a "standard" $\left[{ }^{12} \mathrm{C}\right] /\left[{ }^{13} \mathrm{C}\right]$ abundance ratio (for (U)LIRGs) of $\sim 100$ in NGC 6240. The high ratio found by Papadopoulos et al. (2014) is explained by their attribution of the high- $J$ CO lines to the dense gas phase, whereas our simultaneous fitting and the addition of the $\mathrm{H}^{13} \mathrm{CN}$ and $\mathrm{H}^{13} \mathrm{CO}^{+}$lines/limits leads to the model spontaneously fitting these high $-J$ lines to a 


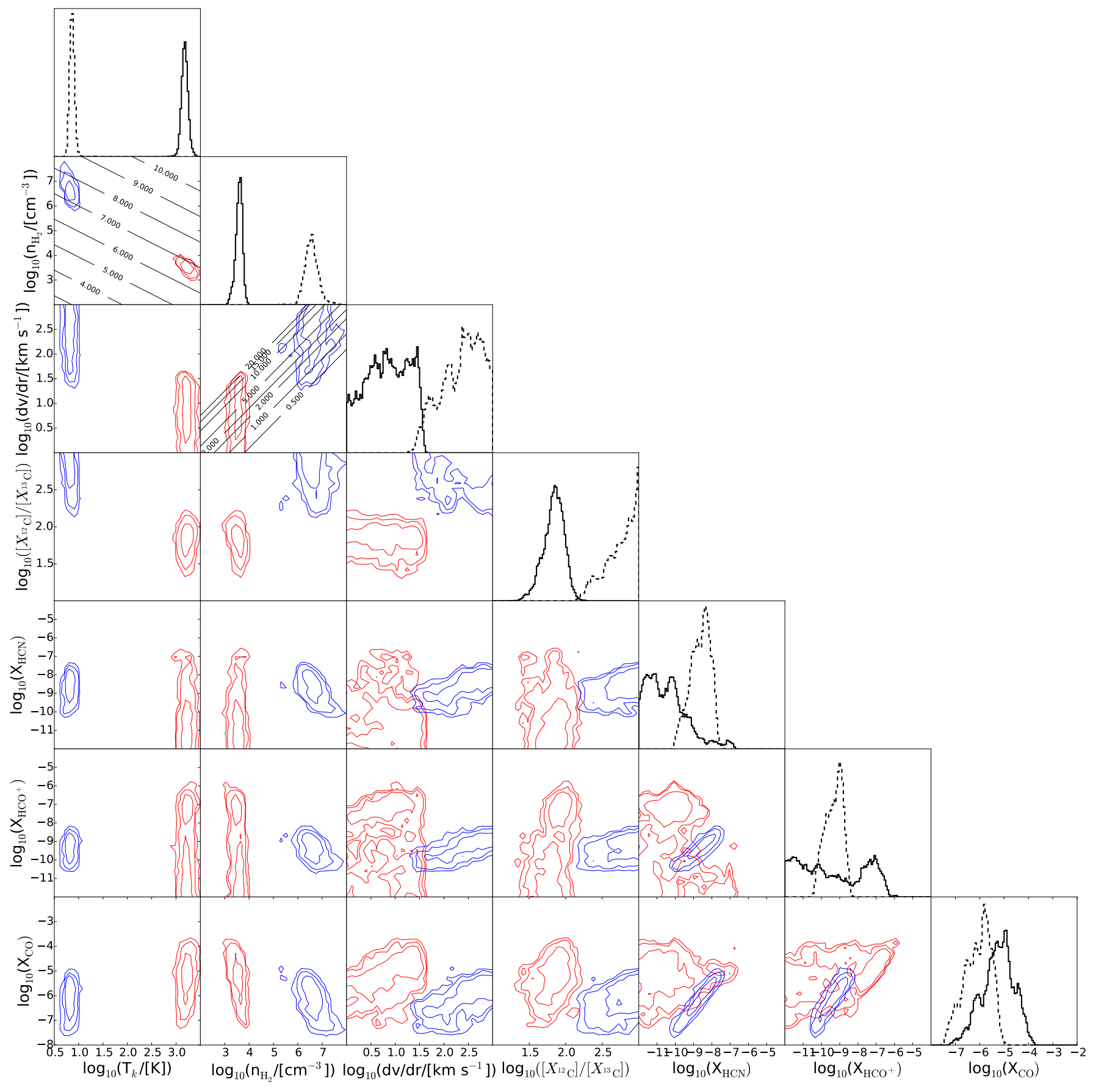

Fig. 15. - The modified step plot for the two phase $\mathrm{HCN}+\mathrm{HCO}^{+}+\mathrm{CO}$ model. Contours are at the $68 \%, 95 \%$ and $99 \%$ credible intervals. Red/solid and blue/dashed contours are phases 1 and 2 , with $T_{\mathrm{bg}}=3$ and $3 \mathrm{~K}$ respectively. Contours of constant pressure have been included in the $T_{\mathrm{k}}-n_{\mathrm{H}_{2}}$ plot at intervals of one dex. There is a clear, robust and spontaneous separation into a hot, diffuse and cold, dense gas phase.

hot, diffuse phase, as did the two phase CO model of Kamenetzky et al. (2014). Our high observed $[\mathrm{HCN}] /\left[\mathrm{H}^{13} \mathrm{CN}\right]$ and $\left[\mathrm{HCO}^{+}\right] /\left[\mathrm{H}^{13} \mathrm{CO}^{+}\right]$ratios are explained by chemical effects (ICE) in the very cold, dense gas. 

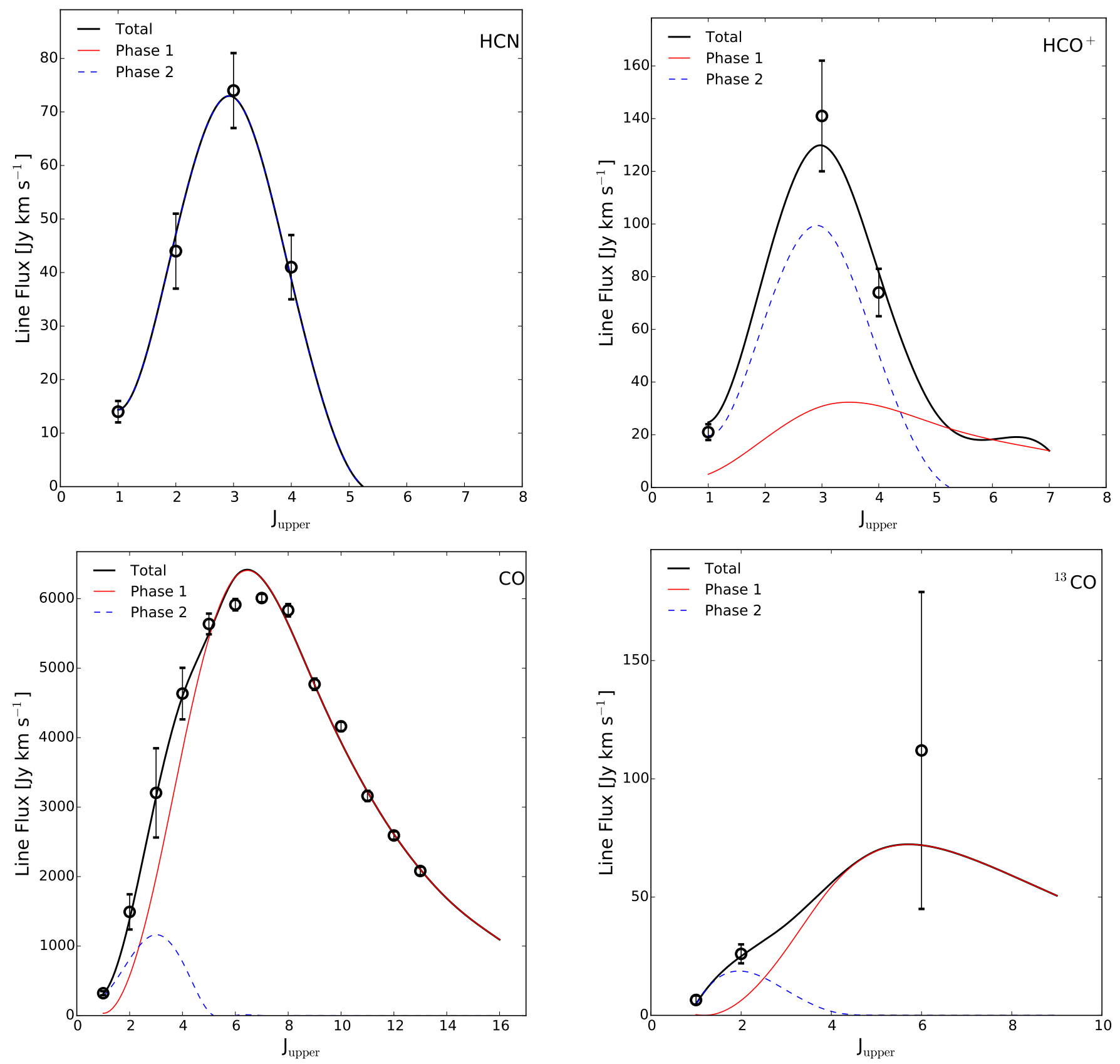

FIG. 16. - SLED fits for the two phase $\mathrm{HCN}+\mathrm{HCO}^{+}+\mathrm{CO}$ model, showing the relative contribution of each gas phase. The same normalisation has been used for the $\mathrm{CO}$ and ${ }^{13} \mathrm{CO}$ SLEDs. 


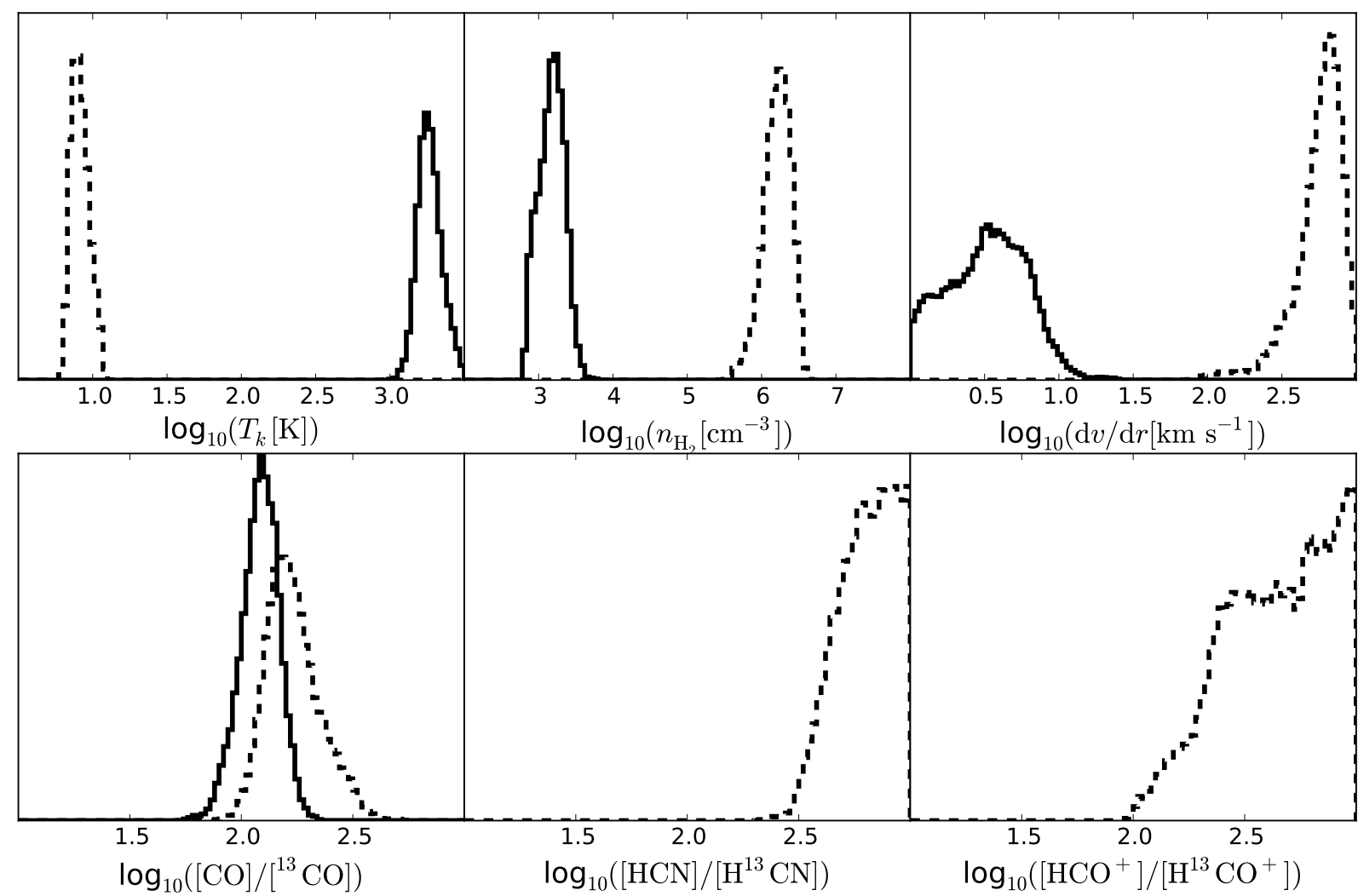

FIG. 17. - 1D marginalised posterior pdfs for the gas phase parameters of the $\left[X_{12} \mathrm{C}\right] /\left[X_{13} \mathrm{C}\right]$ investigatory model. The gas parameters are very similar to those of the $\mathrm{HCN}+\mathrm{HCO}^{+}+\mathrm{CO} 2$ phase model. The $[\mathrm{CO}] /\left[{ }^{13} \mathrm{CO}\right]$ abundance ratios are similar in both phases, and are in the "standard" region for (U)LIRGs, suggesting that the high $[\mathrm{HCN}] /\left[\mathrm{H}^{13} \mathrm{CN}\right]$ and $\left[\mathrm{HCO}^{+}\right] /\left[\mathrm{H}^{13} \mathrm{CO}^{+}\right]$ratios are due to isotope fractionation in the cold gas phase.

TABLE 9

$\left[{ }^{12} \mathrm{C}\right] /\left[{ }^{13} \mathrm{C}\right]$ Study Two Phase Model Solutions. All values $\log _{10}$.

\begin{tabular}{|c|c|c|}
\hline Parameter & Mean & $\min \chi^{2}$ \\
\hline$\overline{T T_{\mathrm{k}, 1} \cdots}$ & $3.3_{3.2}^{3.4}$ & 3.3 \\
\hline$T_{\mathrm{k}, 2}$. & $0.9_{0.9}^{1.0}$ & 0.8 \\
\hline$n_{\mathrm{H}_{2}, 1} \ldots \ldots \ldots \ldots$ & $3.2_{3.0}^{3.4}$ & 3.0 \\
\hline$n_{\mathrm{H}_{2}, 2}$ & $6.2_{6.1}^{6.4}$ & 6.5 \\
\hline$(\mathrm{d} v / \mathrm{d} r)_{1} \ldots \ldots \ldots$ & $0.5_{0.3}^{0.8}$ & 0.3 \\
\hline$(\mathrm{d} v / \mathrm{d} r)_{2} \ldots \ldots \ldots$ & $2.7_{2.7}^{2.9}$ & 3.0 \\
\hline$[\mathrm{CO}] /\left[{ }^{13} \mathrm{CO}\right]_{1} \ldots \ldots$ & $2.1_{2.0}^{2.2}$ & 2.2 \\
\hline$[\mathrm{CO}] /\left[{ }^{13} \mathrm{CO}\right]_{2} \ldots \ldots$ & $2.2_{2.1}^{2.3}$ & 2.3 \\
\hline$[\mathrm{HCN}] /\left[\mathrm{H}^{13} \mathrm{CN}\right] \ldots$ & $2.8_{2.7}^{3.0}$ & 3.0 \\
\hline$\left[\mathrm{HCO}^{+}\right] /\left[\mathrm{H}^{13} \mathrm{CO}^{+}\right]$ & $2.6_{2.6}^{3.0}$ & 3.0 \\
\hline$\cdots \cdots \cdots \cdots$ & $-1.1_{-1.2}^{-1.0}$ & -1.1 \\
\hline
\end{tabular}

